\title{
Algoritmos de Aproximação para Partições Conexas em Grafos
}

\author{
Liliane Rose Benning Salgado
}

\author{
TESE APRESENTADA \\ $\mathrm{AO}$ \\ INSTITUTO DE MATEMÁTICA E ESTATÍSTICA \\ DA \\ UNIVERSIDADE DE SÃO PAULO \\ PARA \\ OBTENÇÃO DO GRAU DE DOUTOR \\ $\mathrm{EM}$ \\ CIÊNCIA DA COMPUTAÇÃO
}

Orientadora: Profa. Dra. Yoshiko Wakabayashi

Durante a elaboração deste trabalho a autora recebeu apoio financeiro da FAPESP

- São Paulo, dezembro de 2003 - 


\title{
Algoritmos de Aproximação para Partições Conexas em Grafos
}

\author{
Liliane Rose Benning Salgado \\ Este exemplar corresponde à redação \\ final da tese devidamente corrigida \\ e defendida por Liliane Rose Benning Salgado \\ e aprovada pela comissão julgadora.
}

São Paulo, 25 de junho de 2004.

Banca examinadora:

- Prof. Dra. Yoshiko Wakabayashi (orientadora) - IME-USP

- Prof. Dra. Cristina Gomes Fernandes - IME-USP

- Prof. Dr. Edson Norberto Cáceres - DCT-UFMS

- Prof. Dra. Kátia Silva Guimarães - CIN-UFPE

- Prof. Dra. Sulamita Klein - IM-UFRJ 

Dedicado a Santíssima Trindade e a Maria, minha Senhora e também minha Mãe. Louvo e agradeço! 



\section{Agradecimentos}

À Profa. Yoshiko Wakabayashi que, muito além de orientadora, foi alguém que me concedeu um apoio incondicional em todos os momentos decisivos da elaboração desta tese. Obrigada por sua compreensão e carinho, sua experiência profissional compartilhada a cada instante engrandeceu muito o meu trabalho.

À FAPESP, pelo apoio financeiro na elaboração desta tese.

Aos Professores Cristina Gomes Fernandes (IME-USP), Edson Cáceres (DCTUFMS), Kátia Silva Guimarães (CIN-UFPE) e Sulamita Klein (COS-UFRJ) por aceitarem compor a banca examinadora desta tese.

Aos professores, funcionários e alunos do Departamento de Ciência da Computação, contemporâneos da minha passagem aqui no Instituto de Matemática e Estatística da USP. Todos que partilharam da minha convivência contribuíram de alguma forma para a conclusão deste projeto de vida. Particularmente, agradeço a Carlinhos, Coelho, Endler, Estela, Fábio, Flávio, Glauber, Jair, José Augusto, Leston, Marcel, Orlando, Renato, Said e Yoshi.

Às minhas amigas, e quase irmãs, Ana Sampaio, Cláudia Abrão, Daniela Vantini, Jeane Melo, Lumena Keller, Márcia Costa, Margarete Rocha e Patrícia Tedesco. Jamais esquecerei o apoio emocional, psicológico e em alguns casos, até financeiro, cedidos nos momentos tão difíceis e decisivos. Espero um dia poder retribuir tudo isto.

Ao Dr. Luís Fernando Maciel, por sua competência profissional e o grande carinho com que tem conduzido a minha recuperação.

À Dra. Sônia Duarte que com competência, experiência, dedicação, carinho e solidariedade tem participado decisivamente na minha recuperação e na finalização desta tese. Além disto, tem me auxiliado cotidianamente a descobrir potencialidades e a investir no auto-conhecimento. Com você tenho aprendido a manter a mente quieta, a espinha ereta e o coração tranquilo em qualquer circunstância da minha vida.

Tudo começa no seio familiar, portanto tudo que sou e conquisto devo e agradeço aos meus queridos pais, José e Miriam Salgado, e às minhas amadas irmãs e respectivas famílias: Nane (in memoriam), Gina, Lu, Maria Eduarda (sobrinha), Kika, Dado (cunhado) e João Pedro (sobrinho). Minha gratidão também à Bibia e à 
Julia pelo extremo cuidado com todos nós.

Neste momento tão especial, registro também o meu carinho a Ana Benning, Luís, Renata, Bruna, Beto, Kátia, Vítor, Carol, Joseana, Natália e Guga. Também agradeço as constantes orações de Luciane. Esta é a família Aleixo que tanto amo.

Finalizo registrando minha enorme gratidão à minha madrinha Gina que me concedeu todo o suporte necessário para a conclusão desta tese. Os obstáculos surgiram e foram superados graças ao seu apoio incondicional. Pessoas assim, que acreditam no desenvolvimento científico e tecnológico deste país são importantes para transformar sonhos em realidade. 


\section{Resumo}

O estudo das propriedades de aproximação de problemas de otimização NPdifíceis é um tópico de interesse tanto da área de otimização quanto da teoria de complexidade computacional. O tema desta tese insere-se neste contexto, dando ênfase ao estudo de problemas de partição de grafos em subgrafos conexos satisfazendo certas especificações. Concentramo-nos no desenvolvimento e análise de algoritmos de aproximação, e questões relativas ao grau de (in)aproximabilidade desses problemas.

Um dos problemas investigados, chamado Max 2-Partição Conexa Balanceada $\left(\mathrm{PCB}_{2}\right)$, é o seguinte: dado um grafo conexo $G=(V, E)$ com uma função peso $w: V \rightarrow \mathbb{Z}_{+}$definida sobre seus vértices, encontrar uma 2-partição $\left(V_{1}, V_{2}\right)$ de $V$ tal que $G\left[V_{1}\right]$ e $G\left[V_{2}\right]$ sejam conexos e o peso do mais 'leve' deles seja o maior possível. Mais formalmente, queremos encontrar uma tal partição que maximiza a função $\min \left\{w\left(V_{1}\right), w\left(V_{2}\right)\right\}$, onde $w(S)$ denota o peso de um conjunto $S$. Exibimos resultados sobre complexidade computacional e inaproximabilidade. Também apresentamos uma releitura de um algoritmo (4/3)-aproximado desenvolvido por Chlebíková [Chl96]. A análise parametrizada que fizemos permite descrever classes de grafos para as quais as soluções devolvidas se aproximam assintoticamente do ótimo. Mostramos que a razão de aproximação desse algoritmo é justa mesmo para grafos 3-conexos. Além disto, elaboramos um algoritmo para grafos 3-conexos usando contrações de arestas, que pode produzir soluções de qualidade melhor do que o algoritmo (4/3)-aproximado. Também apresentamos um esquema de aproximação polinomial para uma classe especial de grafos. Provamos ainda que as versões com e sem pesos do Max 2-Partição Conexa Balanceada possuem o mesmo limiar de aproximação.

Estudamos também uma generalização natural do problema $\mathrm{PCB}_{2}$, denominado Max q-Partição Conexa Balanceada $\left(\mathrm{PCB}_{q}\right)$, para $q>2$. Para o problema $\mathrm{PCB}_{3}$ restrito a grafos 3-conexos exibimos dois algoritmos, sendo um deles uma 2-aproximação. Para o $\mathrm{PCB}_{4}$ restrito a grafos 4-conexos exibimos também uma 2-aproximação, porém apenas sob certas hipóteses sobre os pesos dos vértices.

Investigamos um outro problema, chamado Max Árvore Balanceada, que mostramos ser AP-redutível ao $\mathrm{PCB}_{2}$. Também apresentamos algoritmos de aproximação para um outro problema correlato, denominado $\operatorname{Max}(p / k)$-Bipartição 
Fracionária Conexa. Exibimos um algoritmo para $1 / 2 \leq p / k<1$ cuja razão de aproximação é $3 p /(3 p-k)$. Discutimos brevemente outros problemas correlatos, mencionando alguns resultados encontrados na literatura.

Palavras-chave: algoritmos de aproximação, esquema de aproximação polinomial, grafos $k$-conexos, inaproximabilidade, partição conexa balanceada, partição de grafos. 


\begin{abstract}
In this thesis we study the Max Balanced Connected q-Partition Problem $\left(\mathrm{BCP}_{q}\right)$, defined as follows. Given a connected graph $G=(V, E)$ with a weight function $w: V \rightarrow \mathbb{Z}_{+}$, find a $q$-partition $P=\left(V_{1}, V_{2}, \ldots, V_{q}\right)$ of $V$ such that $G\left[V_{i}\right]$ is connected $(1 \leq i \leq q)$ and $P$ maximizes $\min \left\{w\left(V_{i}\right): 1 \leq i \leq q\right\}$. This problem is known to be NP-hard. We present results on the computational complexity of this problem restricted to special classes of graphs, some non-approximability results, and approximation algorithms. The main results are for $q=2$ and $q=3$, for which we show approximation algorithms with performance ratio at most $4 / 3$ and 2, respectively. We show that $\mathrm{BCP}_{2}$ does not admit an FPTAS even for 2connected graphs, and we exhibit a PTAS for a special class of graphs. We also study some other related problems, showing how some results for $\mathrm{BCP}_{q}$ can be extended to them. For one of these problems, called Max Fractionally Connected $(p / k)$-Bipartition Problem, we exhibit a $3 p /(3 p-k)$-approximation algorithm for $1 / 2 \leq p / k<1$.
\end{abstract}





\section{Índice}

1 Introdução 1

1.1 Problemas Investigados . . . . . . . . . . . . . . . . . 3

1.2 Aplicações . . . . . . . . . . . . . . . . . 5

$\begin{array}{llr}2 & \text { Preliminares } & 9\end{array}$

2.1 Teoria dos Grafos . . . . . . . . . . . . . . . . . . 9

2.2 Complexidade de Problemas de Otimização . . . . . . . . . . . . . 12

2.3 Reduções e Classes de Aproximação . . . . . . . . . . . . . . . . 15

2.4 Qualidade de Aproximação . . . . . . . . . . . . . . . . . . . . 18

2.5 Problemas com Pesos . . . . . . . . . . . . . . . . . . 21

2.6 Estado-da-arte . . . . . . . . . . . . . . . . . . 23

2.6.1 1973-1986: Resultados Dispersos . . . . . . . . . . 23

2.6.2 1987-1990: Classes de Problemas . . . . . . . . . . . . 24

2.6.3 1991-1993: A Conexão PCP . . . . . . . . . . . . . . 26

2.6.4 Resultados Recentes . . . . . . . . . . . . . . . . . . 29

3 Bipartição Conexa Balanceada 33

3.1 Complexidade Computacional . . . . . . . . . . . . . . . . . 34 
$3.1 .1 \quad$ NP-completude . . . . . . . . . . . . . . . . . . 35

3.2 Dificuldade de Aproximação . . . . . . . . . . . . . . . . 40

3.3 Aproximabilidade . . . . . . . . . . . . . . . . . . . 44

3.3.1 Esquema de Aproximação Polinomial . . . . . . . . . . 58

3.3.2 Removendo Pesos . . . . . . . . . . . . . . . . . . . . . 72

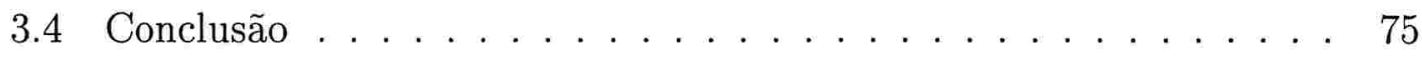

4 Sobre $q$-Partições Conexas Balanceadas $\quad 77$

4.1 Complexidade Computacional . . . . . . . . . . . . . . . 78

4.2 Max 3-Partição Conexa Balanceada . . . . . . . . . . . . . . 81

4.2.1 Grafos 2-conexos e 3-conexos . . . . . . . . . . . 82

4.3 Generalização . . . . . . . . . . . . . . . . . . . . 89

5 Problemas Correlatos $\quad 93$

5.1 O problema Max Árvore Balanceada . . . . . . . . . . . . . 93

5.2 Bipartição Fracionária Conexa . . . . . . . . . . . . . . . 96

5.3 Outros problemas sobre partições conexas . . . . . . . . . . 99

6 Considerações Finais 103

$\begin{array}{ll}\text { Referências Bibliográficas } & 105\end{array}$

Apêndice: Problemas NPO Referenciados. 117 


\section{Lista de Figuras}

2.1 Um grafo e seu correspondente grafo de blocos. . . . . . . . . . . 11

3.1 Exemplo de grafo gerado pela redução. . . . . . . . . . . . . . 36

3.2 Grafo 2-conexo com pesos nos vértices. . . . . . . . . . . . . . . . . 48

3.3 Grafo 2-conexo com pesos nos vértices. . . . . . . . . . . . . . . . . 49

3.4 Grafo 3-conexo com pesos nos vértices. . . . . . . . . . . . . . . . . 54

3.5 Um exemplo comparativo. . . . . . . . . . . . . . . 58

5.1 Uma instância com soluções distintas para as funções (1) e (2). . . . 101 


\section{Lista de Tabelas}

3.1 Classes de grafos para as quais $\mathrm{PCB}_{2}$ sem pesos é NP-difícil. . . . . 35

3.2 Iterações executadas pelo algoritmo BalBicon ${ }_{2} \ldots$. . . . . . . . . . . 48

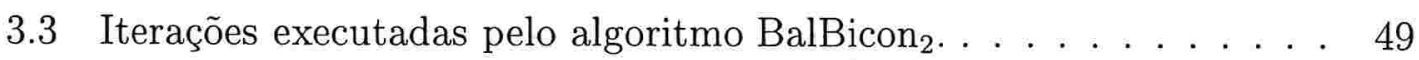

3.4 Iterações executadas pelo algoritmo BalBicon B $_{2} \ldots$. . . . . . . . . 58 


\section{CAPÍtulo 1}

\section{Introdução}

"O homem não é maior do que o resultado de seus pensamentos; o que ele pensa, é o que virá a ser."

(Gandhi)

O estudo de um problema de otimização sob o ponto de vista algorítmico começa invariavelmente pela identificação de sua complexidade computacional. Não raras as vezes, deparamos com problemas que são NP-difíceis, cabendo-nos então decidir que tipo de solução nos interessa: exata, probabilística, ou aproximada.

A busca de tais tipos de solução fazem uso de abordagens, cada uma das quais sacrificam algum aspecto em troca de outro. Soluções exatas, onde eventualmente podemos usar técnicas gerais como branch and bound ou programação dinâmica, sacrificam o uso de recursos computacionais (tempo, espaço) em troca de uma solução exata (ótima). Ainda que esse compromisso pareça natural, nem sempre isso é possível: algumas vezes o tempo exigido é tão grande que apenas instâncias pequenas ou de médio porte são tratáveis na prática.

Soluções probabilísticas, encontradas através do uso de métodos probabilísticos (Monte Carlo, Las Vegas), sacrificam o determinismo (no sentido de que nem sempre as mesmas instâncias produzem as mesmas soluções), mas em geral estes métodos são simples e as soluções são encontradas rapidamente (eventualmente alguma instância pode exigir um tempo bem grande). Há abordagens que devolvem soluções que, com grande probabilidade estão corretas; e há outras que, 
se devolvem uma resposta, esta é correta, porém nem sempre uma tal resposta é devolvida.

Em se tratando de soluções aproximadas, sacrificamos a exatidão em troca de rapidez na busca de tais soluções. Em geral, nos referimos a métodos heurísticos, quando usamos estratégias ad hoc, sem nenhuma garantia quanto à qualidade da solução encontrada. Dependendo do problema, este tipo de abordagem pode eventualmente encontrar soluções de boa qualidade (só comprovável através de amplos testes computacionais). Em contrapartida a este tipo de abordagem que carece de um 'selo de qualidade', existem os chamados algoritmos de aproximação. Neste caso, além da rapidez, tem-se uma garantia quanto à qualidade da solução encontrada (em comparação com a solução exata). Quando conseguimos tanto rapidez (polinomialidade) quanto boa qualidade, temos uma situação altamente desejável, tanto em termos práticos (quando se trata de um problema com muitas aplicações) quanto em termos teóricos. No aspecto teórico, a relevância resulta do fato de que muitas vezes são desenvolvidas ferramentas (técnicas de prova) que além de serem interessantes por si só, podem também ser usadas em outros contextos; ou que certos aspectos desconhecidos sejam revelados com relação ao problema em foco, levando a indagações e resultados de natureza mais geral.

Nas últimas duas décadas o estudo de algoritmos de aproximação para problemas combinatórios NP-difíceis tem sido tema central de pesquisa em otimização combinatória. O número de trabalhos sobre este assunto tem crescido substancialmente, com a introdução e formalização de novas técnicas para melhoria das garantias de aproximação [CK98, Hoc97, MPS98, $\mathrm{ACG}^{+} 99$, Vaz00].

A teoria de complexidade computacional clássica, desenvolvida por Cook [Coo71] e Karp [Kar72], fornece um modelo computacional robusto para problemas de decisão, possibilitando o desenvolvimento de uma rica teoria da complexidade computacional baseada em reduções de tempo polinomial, e a obtenção de várias classes de problemas.

$\mathrm{Na}$ tentativa de estabelecer um tal ambiente teórico para o estudo da aproximabilidade de problemas de otimização, foram definidos formalmente os chamados Problemas de Otimização NP, denotados por NPO, e pesquisas têm sido direcionadas para entender e classificar tais problemas.

É bem conhecido que se um problema NPO pode ser resolvido em tempo polinomial, então seu problema de decisão correspondente pode também ser resolvido 
da mesma maneira. Assim, qualquer problema NPO cuja versão de decisão é um problema NP-completo não pode ser resolvido em tempo polinomial, sob a hipótese de que $\mathrm{P} \neq \mathrm{NP}$. Nestes casos, é natural sacrificar a otimalidade e buscar soluções aproximadas computáveis em tempo polinomial.

Há que se ressaltar porém que existem limites quanto ao grau de aproximabilidade de certos problemas, a menos que $\mathrm{P}=\mathrm{NP}$. Constatou-se que para muitos problemas de otimização, encontrar soluções aproximadas é tão difícil quanto encontrar uma solução exata; além disso, estes podem apresentar propriedades de aproximação drasticamente diferentes.

Os tópicos estudados nesta tese inserem-se no contexto de estudos sobre complexidade e aproximabilidade de certos problemas sobre partição de grafos em subgrafos conexos. Investigamos a complexidade computacional desses problemas e outros correlatos, projetamos algoritmos de aproximação, e pesquisamos questões relativas ao grau de aproximabilidade dos mesmos.

Definimos na próxima seção os problemas estudados e mencionamos os resultados que foram obtidos. Na última seção mencionamos alguns problemas práticos que podem ser modelados como problemas de partição de grafos.

\subsection{Problemas Investigados}

O tema central desta tese é o estudo do problema Max $q$-Partição Conexa Balanceada $\left(\mathrm{PCB}_{q}\right)$, onde $q$ é um inteiro maior ou igual a 2. Neste problema, a entrada é um grafo conexo $G=(V, E)$ com uma função peso $w: V \rightarrow \mathbb{Z}_{+}$definida sobre seus vértices, e o objetivo é encontrar uma $q$-partição $\left(V_{1}, V_{2}, \ldots, V_{q}\right)$ de $V$ tal que o subgrafo induzido por cada um destes conjuntos $V_{i}$ é conexo e o peso do mais 'leve' deles é tão grande quanto possível.

No Capítulo 3 estudamos o problema $\mathrm{PCB}_{2}$. Do ponto de vista algorítmico, é suficiente resolver o $\mathrm{PCB}_{2}$ para grafos 2-conexos, já que algoritmos para tais grafos podem ser usados para resolver este problema quando o grafo de entrada é conexo. Assim, é interessante obter resultados sobre o $\mathrm{PCB}_{2}$ restrito a grafos 2-conexos.

Nesta direção, mostramos que, sob a hipótese de que $\mathrm{P} \neq \mathrm{NP}$, a versão de decisão do $\mathrm{PCB}_{2}$ restrito a grafos 2-conexos é um problema fortemente NP-completo, e portanto não admite um FPTAS. Um outro resultado de inaproximabilidade que 
provamos na Seção 3.2 é que o $\mathrm{PCB}_{2}$ sobre grafos conexos com $n$ vértices não admite um algoritmo $\left(1+\epsilon\right.$ )-aproximado onde $\epsilon \leq \frac{1}{n^{2}}$, a menos que $\mathrm{P}=\mathrm{NP}$ (mesmo para grafos bipartidos). Uma questão natural que surge neste contexto é se este problema admite um PTAS. Na tentativa de responder esta pergunta obtivemos duas classes de grafos para as quais exibimos um PTAS. No caso geral, não sabemos uma resposta.

Na Seção 3.3 apresentamos uma releitura do algoritmo (4/3)-aproximado desenvolvido por Chlebíková [Chl96]. A análise parametrizada que efetuamos permite descrever classes de grafos para as quais as soluções encontradas se aproximam assintoticamente do ótimo. Adicionalmente, mostramos que a razão de aproximação deste algoritmo é justa mesmo para grafos 3-conexos. Além disto, apresentamos para grafos 3-conexos um algoritmo baseado em contrações de arestas que pode devolver soluções com qualidade melhor do que a razão $4 / 3$ garantida pelo algoritmo anterior.

Na Seção 3.3.1 exibimos um PTAS para o $\mathrm{PCB}_{2}$ restrito a duas classes de grafos que chamamos de $\Gamma$-grafos e $C_{w}$-grafos. Nossa motivação para estudar estes grafos advém de algumas topologias de redes de longa distância (wide area networks) que são casos especiais desta classe de grafos. O esquema de aproximação que apresentamos é baseado nas idéias usadas no FPTAS para o problema Max 0/1 Mochila, desenvolvido por Ibarra, Kim [IK75] e Lawler [Law79], combinadas com idéias de um algoritmo $\frac{9}{8}$-aproximado exibido por Galbiati et al. [GMM97]. Até onde sabemos, nosso algoritmo é o primeiro a aplicar estas idéias para os problemas de partição estudados nesta tese.

$\mathrm{Na}$ Seção 3.3.2 mostramos que as versões com e sem pesos do $\mathrm{PCB}_{2}$ (para grafos conexos) possuem o mesmo limiar de aproximação.

No Capítulo 4 investigamos o problema Max $q$-Partição Conexa Balanceada $\left(\mathrm{PCB}_{q}\right)$, onde $q \geq 3$. Na Seção 4.1 analisamos a complexidade do $\mathrm{PCB}_{q}$ e listamos os casos polinomiais e NP-difíceis relacionados com este problema. Também comentamos que os únicos algoritmos de aproximação conhecidos para o $\mathrm{PCB}_{q}$ são restritos às grades $G_{m \times n}$, para $n \geq 3$. Esses algoritmos porém, não têm uma razão de aproximação que possa ser explicitada independente do resultado da execução desses algoritmos.

$\mathrm{Na}$ Seção 4.2 estudamos o $\mathrm{PCB}_{3}$. Neste caso, restringimos nossa atenção aos grafos 2-conexos e 3-conexos. Além de provarmos a intratabilidade do $\mathrm{PCB}_{3}$ para 
estes casos, desenvolvemos algoritmos de aproximação. Um dos algoritmos exibidos para grafos 3-conexos é uma 2-aproximação. Na Seção 4.3 investigamos o $\mathrm{PCB}_{q}$ restrito a grafos $q$-conexos, $q \geq 4$. Para o caso $q=4$ exibimos um algoritmo 2-aproximado para algumas instâncias.

No Capítulo 5 investigamos outros problemas relacionados com o $\mathrm{PCB}_{q}$. Na Seção 5.1 estudamos o problema Max Árvore Balanceada, que mostramos ser APredutível ao $\mathrm{PCB}_{2}$. Também estudamos o problema Max $(p / k)$-Bipartição Fracionária Conexa $\left(\mathrm{BFC}_{p / k}\right)$, definido para $1 \leq p<k$. Neste problema, definido sobre instâncias como a do $\mathrm{PCB}_{2}$, o objetivo é encontrar uma 2-partição conexa $\left(V_{1}, V_{2}\right)$ do grafo de entrada tal que $w\left(V_{1}\right)$ é máximo sujeito a $w\left(V_{1}\right) \leq(p / k) w(V)$. Este problema pode ser visto como uma generalização do $\mathrm{PCB}_{2}$, já que $\mathrm{PCB}_{2}$ é equivalente ao $\mathrm{BFC}_{1 / 2}$. Na Seção 5.2 exibimos algoritmos de aproximação para este problema. Outros problemas correlatos são discutidos na última seção deste capítulo.

No Capítulo 6 tecemos algumas considerações finais sobre o trabalho desenvolvido nesta tese.

No Apêndice apresentamos a definição de todos os problemas de otimização mencionados nesta tese.

\subsection{Aplicações}

Mencionamos nesta seção alguns problemas práticos que podem ser modelados como problemas de partição conexa balanceada.

A primeira aplicação é sobre um problema de um órgão público que precisa fazer uma subdivisão balanceada de um terreno, constituído de vários lotes. Tal terreno é uma área de mineração retangular, que deve ser distribuída a $q$ companhias. Esta subdivisão deve ser tal que cada companhia opere livremente em seus próprios lotes (isto é, cada conjunto de lotes deve ser conexo) e nenhuma companhia deve ter uma probabilidade de sucesso muito baixa. Para isso, foi feita - através de prospecção geológica - uma estimativa da probabilidade de descobrir diamantes em cada lote. Dessa forma, para ser mais justo, o órgão público deseja fazer uma subdivisão que maximize a probabilidade mínima de descobrir diamantes em cada conjunto de lotes. Neste caso, temos uma aplicação do $\mathrm{PCB}_{q}$, cujo grafo de entrada é uma 
grade, e o peso de seus vértices é uma estimativa de probabilidade [BLLS98].

Outra situação onde o particionamento de grades $G_{m \times 2}$ (escadas) pode ser utilizado é no balanceamento de tarefas de supervisão em uma linha de montagem de circuitos eletrônicos [BLLS01]. A linha de produção precisa produzir dois tipos diferentes de placas: A e B. Placas do tipo A e do tipo B utilizam diferentes seqüências de operações que são realizadas por máquinas que devem ter seus componentes alterados para realizar cada uma dessas tarefas. A supervisão da linha de montagem é executada por um dado número de computadores chamados supervisores. Cada supervisor cuida de um subconjunto de operações (que podem levar tempos bem variados) e é responsável pela troca de componentes entre máquinas diferentes. Deseja-se balancear a tarefa de supervisão entre um dado número $q$ de supervisores. Visto que a carga de trabalho devido a transferência de componentes é maior quando as máquinas pertencem a diferentes supervisores, para minimizar o gerenciamento da transferência de componentes entre supervisores, deseja-se considerar somente partições conexas.

Uma outra aplicação interessante é em sistemas operacionais [BP95]. Considere uma árvore enraizada onde os vértices representam procedimentos; sendo que existe uma aresta de um vértice $A$ para um vértice $B$ se o procedimento $A$ chama o procedimento $B$. O peso de um vértice representa a quantidade de memória exigida para alocar o procedimento correspondente. No caso, a soma de memória exigida para todos os procedimentos é maior do que o espaço disponível na memória principal, sendo então necessário um sistema de paginação. Um sistema de paginação é uma partição dos procedimentos em conjuntos disjuntos chamados páginas. Somente algumas páginas podem estar na memória simultaneamente, devido às limitações de espaço, as demais são armazenadas em memória secundária. Páginas são trocadas entre as memórias principal e secundária de acordo com a utilização de seus procedimentos na hora da execução. As operações de troca são lentas, devido às operações de entrada e saída que precisam ser feitas na memória secundária. Assim, para reduzir a quantidade destas operações, objetiva-se armazenar procedimentos que são chamados um pelo outro na mesma página. Ou seja, o objetivo é particionar a árvore em $q$ subárvores, e definir para cada página uma tal subárvore.

O tamanho da memória de uma página deve ser suficientemente grande para armazenar todos os seus procedimentos. Visto que páginas diferentes são trocadas com outras (na memória principal), devemos alocar para cada página a quantidade de memória da página de maior tamanho, para permitir trocas entre quaisquer 
páginas. Neste caso, desejamos minimizar a quantidade de memória da página com o máximo tamanho. Ou seja, buscamos uma $q$-partição onde a classe com o maior tamanho seja a menor possível. Se considerarmos a função objetivo do $\mathrm{BCP}_{q}$, isto é, se buscarmos uma $q$-partição conexa que maximiza o tamanho da menor classe (max-min), é possível que o tamanho da maior página possa ser maior do que a da solução encontrada no caso anterior (min-max), mas ainda assim estaremos garantindo uma partição balanceada. No Capítulo 5 comentamos essas duas funções objetivo.

Mencionamos outras aplicações nos capítulos 3 e 4 deste trabalho. 


\title{
CAPÍtulo 2
}

\section{Preliminares}

\author{
"Na matemática você não compreende coisas. \\ Você simplesmente se acostuma com elas." \\ (Johann von Neumann)
}

Neste trabalho supomos que o leitor esteja familiarizado com conceitos da teoria de complexidade computacional e da teoria dos grafos. Na primeira seção deste capítulo apresentamos alguns conceitos sobre grafos com a finalidade de estabelecer a terminologia e a notação adotadas. Na seção seguinte revisamos algumas definições básicas e resultados sobre complexidade e aproximabilidade de problemas de otimização NP, seguida de um breve resumo sobre o desenvolvimento da área.

\subsection{Teoria dos Grafos}

Um grafo $G=(V, E)$ é um par ordenado que consiste de um conjunto finito não-vazio $V$ de vértices e um conjunto $E$ de pares não-ordenados de elementos distintos de $V$, chamados arestas. O conjunto de vértices de $G$ também é denotado por $V(G)$ e o seu conjunto de arestas por $E(G)$.

Se uma aresta $e$ é definida por um par de vértices, digamos $\{u, v\}$, escrevemos $e=\{u, v\}$, ou simplesmente $e=u v$, e dizemos que os vértices $u$ e $v$ são os seus extremos. Também dizemos que esses vértices são vizinhos ou adjacentes, e que a 
aresta $e$ é incidente ao vértice $u$ (e ao vértice $v$ ).

A vizinhança de um vértice $v$ num grafo $G$, denotada por $N_{G}(v)$, é o conjunto dos vértices de $G$ que são vizinhos de $v$. Quando o grafo $G$ está claro pelo contexto, escrevemos simplesmente $N(v)$.

Um grafo $G$ é trivial se tem apenas um vértice; e $G$ é completo se todos os seus vértices são dois a dois adjacentes. Denotamos por $K_{n}$ um grafo completo com $n$ vértices. Um grafo é bipartido se seu conjunto de vértices é da forma $X \cup Y$, com $X \cap Y=\emptyset$, e suas arestas têm um extremo em $X$ e outro em $Y$.

O grau de um vértice $v$ num grafo $G$, denotado por $g(v)$, é o número de arestas de $G$ incidentes a $v$. O grau máximo de $G$, denotado por $\Delta(G)$, é definido como $\Delta(G)=\max \{g(v) \mid v \in V\}$.

Um caminho em um grafo $G$ é uma seqüência de vértices distintos $P=$ $\left(v_{0}, \ldots, v_{k}\right)$ tal que $v_{i} v_{i+1} \in E(G)$ para todo $i, 0 \leq i<k$. Neste caso dizemos que $V(P)=\left\{v_{0}, \ldots, v_{k}\right\}$ e $E(P)=\left\{v_{i} v_{i+1} \mid i=0, \ldots, k-1\right\}$. O comprimento do caminho $P$ é o número de arestas em $E(P)$. Dizemos que o caminho $P$ vai do vértice $v_{0}$ ao vértice $v_{k}$, ou que $P$ é um caminho entre $v_{0}$ e $v_{k}$. Se $P$ é o caminho acima e $v_{k} v_{0}$ é uma aresta de $G$ então $C=\left(v_{0}, v_{1}, \ldots, v_{k}, v_{0}\right)$ é um circuito em $G$.

Um grafo $G$ é conexo se entre quaisquer dois de seus vértices existe um caminho.

Uma floresta é um grafo que não contém circuitos. Uma árvore é uma floresta conexa. Os vértices de grau 1 em uma árvore são chamados folhas.

Sejam $G=(V, E)$ e $G^{\prime}=\left(V^{\prime}, E^{\prime}\right)$ dois grafos. Dizemos que $G^{\prime}$ é um subgrafo de $G$ se $V^{\prime} \subseteq V$ e $E^{\prime} \subseteq E$. Dizemos que $G^{\prime}$ é um subgrafo gerador de $G$ se $V^{\prime}=V$. Uma árvore geradora de um grafo $G$ é um subgrafo gerador de $G$ que é uma árvore. Se $X \subseteq V$ então o subgrafo de $G$ induzido por $X$, denotado por $G[X]$, é o subgrafo de $G$ com conjunto de vértices $X$ e conjunto de arestas $\{u v \in E(G) \mid u, v \in X\}$.

Se $G$ é um grafo e $X$ é um subconjunto de vértices de $G$, então $G-X$ é o subgrafo de $G$ obtido pela remoção de todos os vértices em $X$ e de todas as arestas incidentes a $X$. Se $X=\{x\}$ é um conjunto unitário, escrevemos $G-x$ em vez de $G-\{x\}$. Para um subconjunto $F \subseteq E$, temos que $G-F=(V, E \backslash F)$. Como no caso de vértices, se $e$ é uma aresta então escrevemos $G-e$ em vez de $G-\{e\}$.

Os subgrafos conexos maximais de um grafo $G$ são chamados componentes conexos. Denotamos por $c(G)$ o número de componentes conexos de $G$. 

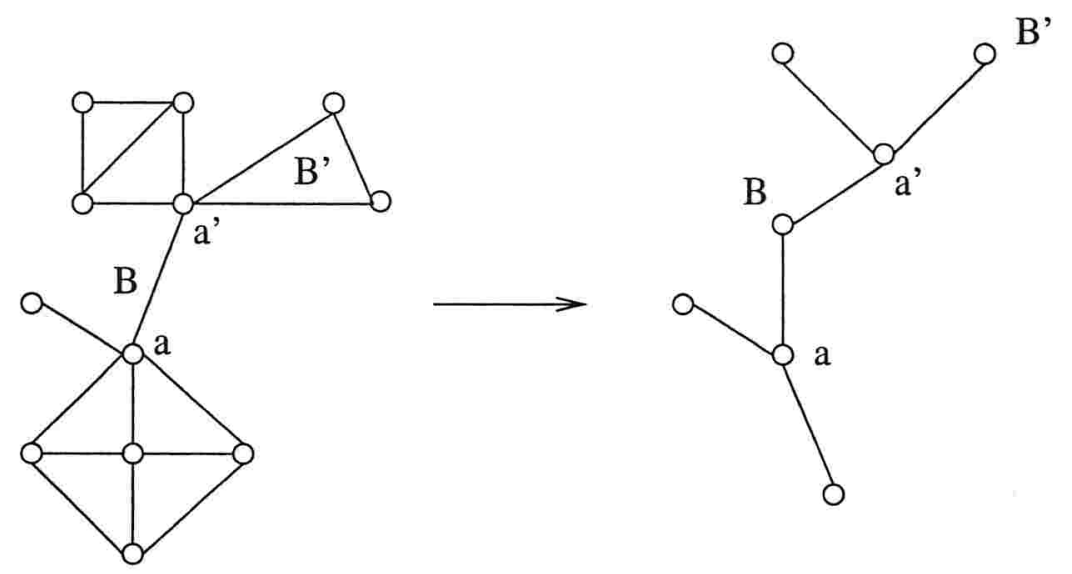

Figura 2.1: Um grafo e seu correspondente grafo de blocos.

Num grafo $G=(V, E)$ um subconjunto $X \subseteq V$ é chamado um corte de vértices de $G$ se $c(G-X)>c(G)$. Se $X=\{v\}$ então dizemos que $v$ é um ponto de articulação de $G$.

Seja $G=(V, E)$ um grafo conexo e $k$ um natural. Dizemos que $G$ é $k$-conexo se $G-X$ é conexo para todo $X \subseteq V$ tal que $|X|<k$. A conectividade de $G$ é o menor natural $k$ tal que $G$ é $k$-conexo. Se $G$ é 2 -conexo também dizemos que $G$ é biconexo.

Os subgrafos maximais de um grafo $G$ que são 2-conexos, ou isomorfos a $K_{2}$, são chamados blocos de $G$. A maneira como os blocos de um grafo $G$ se intersectam revelam muito sobre a estrutura do grafo. Tal estrutura pode ser definida através de um grafo, do seguinte modo. Seja $A$ o conjunto dos pontos de articulação de $G$ e $B$ o conjunto de seus blocos. O grafo de blocos de $G$ é um grafo bipartido com conjunto de vértices $A \cup B$ e conjunto de arestas da forma $a B$, onde $a \in B$. A Figura 2.1 exibe um grafo e o seu correspondente grafo de blocos. Observe que o grafo de blocos de um grafo conexo é uma árvore.

Finalizamos esta seção apresentando um conceito que é central nesta tese.

Seja $A$ um conjunto e $A_{1}, A_{2}, \ldots, A_{q}$ subconjuntos não-vazios de $A$. Dizemos que $P=\left(A_{1}, A_{2}, \ldots, A_{q}\right)$ é uma partição de $A$ se $A=A_{1} \cup A_{2} \cup \ldots \cup A_{q}$ e $A_{i} \cap A_{j}=\emptyset$ para $1 \leq i<j \leq q$. Também dizemos que $P$ é uma $q$-partição de $A$.

Se $G$ é um grafo conexo e $P=\left(V_{1}, \ldots, V_{q}\right)$ é uma $q$-partição de $V(G)$ tal que $G\left[V_{i}\right]$ é conexo para $1 \leq i \leq q$, dizemos que $P$ é uma $q$-partição conexa de $G$. 


\subsection{Complexidade de Problemas de Otimização}

Nesta seção vemos como a teoria da complexidade de problemas de decisão pode ser estendida para problemas de otimização. Inicialmente, discutimos as relações entre suas versões construtiva, de decisão e de avaliação. Ressaltamos que as definições e os teoremas apresentados aqui seguem de perto a terminologia usada por Ausiello et al. $\left[\mathrm{ACG}^{+99}\right.$.

É importante observar que qualquer problema de otimização $\mathcal{P}$ tem um problema de decisão $\mathcal{P}_{D}$ associado. Quando $\mathcal{P}$ é um problema de minimização (maximização), $\mathcal{P}_{D}$ verifica, para algum $k>0$, a existência de uma solução viável $y$ da instância $x$ com valor $m(x, y) \leq k(m(x, y) \geq k)$.

Além disto, problemas construtivos e de avaliação podem também ser associados a um problema de otimização $\mathcal{P}$. No caso de um problema construtivo $\mathcal{P}_{C}$, o objetivo é, dada uma instância $x$, encontrar uma solução ótima $y^{*}$ para $x$ e sua medida $m\left(x, y^{*}\right)$. Na versão de avaliação $\mathcal{P}_{A}$, dada uma instância $x$, o objetivo é determinar o valor da medida ótima $m\left(x, y^{*}\right)$.

Note que, para qualquer problema de otimização $\mathcal{P}$, a versão de decisão $\mathcal{P}_{D}$ não é mais difícil do que o problema construtivo $\mathcal{P}_{C}$. De fato, para responder $\mathcal{P}_{D}$ sobre uma instância $x$ é suficiente utilizar algum algoritmo para $\mathcal{P}_{C}$, obtendo assim uma solução ótima $y^{*}$ e o seu valor $m\left(x, y^{*}\right)$; feito isso, é suficiente verificar se $m\left(x, y^{*}\right) \leq k$ (minimização) ou $m\left(x, y^{*}\right) \geq k$ (maximização).

A forma mais direta para caracterizar a complexidade de problemas de otimização é considerar o tempo necessário para resolvê-los e estender para os problemas de otimização a teoria desenvolvida para os problemas de decisão.

Um aspecto relevante nesses estudos é caracterizar problemas de otimização $\mathcal{P}$ que podem ser considerados tratáveis, isto é, para os quais existe um algoritmo (de tempo) polinomial que, para qualquer instância $x$ devolve uma solução ótima $y^{*}$ e o seu valor $m\left(x, y^{*}\right)$. Estamos interessados no estudo das versões construtivas de problemas de otimização que residem no limite entre tratabilidade e intratabilidade. Estes, por analogia com problemas de decisão NP, são chamados de NPO, denominamos também de NPO a classe de tais problemas.

Definição 2.2.1. Um problema $\mathcal{P}$ de otimização NP $(\mathrm{NPO})^{1}$ é uma 4-upla $(I$, sol,m,opt) tal que

\footnotetext{
${ }^{1}$ Esta abreviação é oriunda do termo em inglês, "NP Optimization Problems". Decidimos não
} 
- I, o conjunto de instâncias de $\mathcal{P}$, é reconhecível em tempo polinomial.

- Dada uma instância $x \in I$, sol(x) denota o conjunto das soluções viáveis de $x$. Existe um polinômio $p$ tal que, para qualquer $x$ e para qualquer $y \in \operatorname{sol}(x)$, $|y| \leq p(|x|)$. Para qualquer instância $x$ e qualquer y tal que $|y| \leq p(|x|)$, é decidivel em tempo polinomial se $y \in \operatorname{sol}(x)$. (Se $x$ é uma instância, $|x|$ denota o tamanho de $x$.)

- Dada uma instância $x$ e uma solução viável y de $x, m(x, y)$ denota a medida inteira positiva de y (freqüentemente chamada o valor de y). A função $m$ é computável em tempo polinomial, sendo também chamada função objetivo.

- opt $\in\{\max , \min \}$ indica o objetivo do problema: dada uma instância $x$, encontrar uma solução y de $x$ cuja medida é máxima ou mínima dependendo se o problema é de maximização (opt $=$ max) ou minimização (opt $=$ min). Uma tal solução é chamada solução ótima e o seu valor (chamado ótimo) é denotado por opt $(x)$.

Observação 2.2.1. Quando for conveniente, para nos referirmos a qualquer dos elementos da 4-upla (I,sol, m,opt) de um problema $\mathcal{P}$, usaremos o nome do problema como subscrito: $I_{\mathcal{P}}$, sol $l_{\mathcal{P}}, m_{\mathcal{P}}$, opt $t_{\mathcal{P}}$.

Observe que com a definição de problemas NPO podemos associar um modelo de computação não-determinístico. Isto é formalmente apresentado no seguinte resultado que mostra que a classe NPO, no contexto de otimização, é naturalmente análoga à classe NP.

Teorema 2.2.1. Para qualquer problema de otimização $\mathcal{P}$ em NPO, o problema de decisão correspondente $\mathcal{P}_{D}$ pertence a NP.

A relação entre as classes NP e NPO, que acontece no caso de computações nãodeterminísticas, pode ser traduzida, no caso de algoritmos determinísticos, pela seguinte definição, que exibe a classe de problemas NPO cujas versões construtivas são solúveis eficientemente.

Definição 2.2.2. Um problema de otimização $\mathcal{P}=(I$, sol, $m$,opt $)$ pertence à classe PO se está em NPO e existe um algoritmo $A$ polinomial que, para qualquer instância $x \in I$, devolve uma solução ótima $y^{*} \in \operatorname{sol}(x)$ e seu valor $m\left(x, y^{*}\right)$.

traduzi-la devido a denominação ser muito comum na literatura da área. 
É desconhecida a complexidade intrínseca dos problemas de otimização em NPO cuja pertinência a PO não foi estabelecida. Analogamente ao caso dos problemas de decisão, para esses problemas nenhum algoritmo polinomial foi descoberto mas nenhuma prova de intratabilidade é conhecida ainda. De fato, a questão "PO = NPO?" está estritamente relacionada à questão "P = NP?", visto que foi provado que as duas questões são equivalentes no sentido que uma resposta positiva para uma delas implica uma resposta positiva para a outra.

Observe que se a versão de decisão $\mathcal{P}_{D}$ de um problema $\mathcal{P}$ em NPO é um problema NP-completo, então $\mathcal{P}$ é NP-difícil. Claramente, um problema de decisão $\mathcal{P}_{D}$ poderia ser facilmente resolvido se um oráculo encontrasse uma solução do problema de otimização construtivo $\mathcal{P}_{C}$.

O fato de um problema $\mathcal{P}$ em NPO ser NP-difícil naturalmente coloca $\mathcal{P}$ no mais alto nível de complexidade na classe NPO, como acontece em NP com os problemas NP-completos.

Nesta análise da complexidade de problemas de otimização, destacamos como mais um aspecto importante as relações mútuas existentes entre as várias versões (decisão, avaliação e construtiva) de um problema de otimização.

Antes de abordá-las definiremos a redução de tempo polinomial de Turing usada nos resultados mencionados a seguir.

Definição 2.2.3. Dados dois problemas de otimização $\mathcal{P}$ e $\mathcal{P}^{\prime}$, dizemos que $\mathcal{P}$ é redutivel a $\mathcal{P}^{\prime}$, em símbolos $\mathcal{P} \leq_{T}^{p} \mathcal{P}^{\prime}$, se $\mathcal{P}$ pode ser resolvido em tempo polinomial por um algoritmo que usa um oráculo que, para qualquer instância $x \in I_{\mathcal{P}^{\prime}}$, devolve uma solução ótima $y^{*}$ de $x$ e seu valor $m\left(x, y^{*}\right)$.

Definição 2.2.4. Dados dois problemas de otimização $\mathcal{P}$ e $\mathcal{P}^{\prime}$, dizemos que $\mathcal{P} e$ $\mathcal{P}^{\prime}$ são equivalentes, em símbolos $\mathcal{P} \equiv_{T}^{p} \mathcal{P}^{\prime}$, se $\mathcal{P} \leq_{T}^{p} \mathcal{P}^{\prime}$ e $\mathcal{P}^{\prime} \leq_{T}^{p} \mathcal{P}$.

Teorema 2.2.2. Se $\mathcal{P}$ é um problema em NPO, então $\mathcal{P}_{\mathcal{D}} \equiv_{T}^{p} \mathcal{P}_{A} \leq_{T}^{p} \mathcal{P}_{C}$.

Teorema 2.2.3. Se $\mathcal{P}$ é um problema NPO tal que $\mathcal{P}_{D}$ é $N P$-completo, então temos que $\mathcal{P}_{C} \leq_{T}^{p} \mathcal{P}_{D}$.

Permanece em aberto se existe algum problema NPO cuja versão construtiva é mais difícil do que a sua versão de avaliação.

Neste contexto de problemas de otimização NP-difíceis, discutimos as diferentes propriedades de aproximação que estes problemas podem apresentar. Estes dife- 
rentes graus de aproximabilidade detectados motivaram a definição de classes de aproximação e reduções que preservam aproximação, na intenção de desenvolver um ambiente teórico similar àquele da teoria da NP-completude. Este é o assunto da próxima seção.

\subsection{Reduções e Classes de Aproximação}

Máquinas de Turing não determinísticas de tempo polinomial fornecem um modelo computacional robusto para problemas de decisão, possibilitando o desenvolvimento de uma rica teoria da complexidade estrutural baseada em reduções de tempo polinomial e a obtenção de várias classificações de problemas NP. No entanto, apesar das muitas tentativas para classificar os problemas de otimização e estudar suas propriedades estruturais, constata-se a falta de modelos computacionais robustos para estes problemas, impedindo a elaboração de uma teoria da complexidade estrutural similar.

Um exemplo disto é que apesar de todos os problemas NP-completos serem redutíveis entre si, as reduções polinomiais, conhecidas do contexto da NPcompletude, raramente preservam a função objetivo e mesmo quando isto acontece não é garantida a conservação das características de aproximação.

A situação acima é bem caracterizada pelos problemas Max Conjunto Independente e Min Cobertura de Vértices. Os dois problemas são trivialmente redutíveis entre si já que $I$ é um conjunto independente de um grafo $G=(V, E)$ se e somente se $V-I$ é uma cobertura de vértices do mesmo grafo. Contudo, esses problemas possuem propriedades de aproximação completamente diferentes: Max Conjunto Independente não é aproximável dentro de $|V|^{1 / 4-\delta}$ para qualquer $\delta$ [BGS98], enquanto Min Cobertura de Vértices tem uma 2-aproximação.

Em conseqüência, visando resultados de completude em classes de aproximação surge a necessidade de definirmos reduções que preservam aproximação.

Definição 2.3.1. Uma redução que preserva a aproximação de um problema de otimização $\mathcal{P}$ para outro $\mathcal{P}^{\prime}$, transforma:

- cada instância de $\mathcal{P}$ para uma instância de $\mathcal{P}^{\prime}$, e 
- cada solução desta instância de $\mathcal{P}^{\prime}$ para uma solução daquela original, preservando a aproximação.

Dados $\mathcal{P}$ e $\mathcal{P}^{\prime}$ como na definição acima, se existe um algoritmo de aproximação $A^{\prime}$ para $\mathcal{P}^{\prime}$ é possível utilizá-lo para conseguir um algoritmo de aproximação para $\mathcal{P}$. De fato, suponha que $x$ é uma instância de $\mathcal{P}$. Então primeiramente transformamos $x$ em uma instância $t^{\prime}(x)$ de $\mathcal{P}^{\prime}$. Depois, aplicamos o algoritmo de aproximação $A^{\prime}$ de $\mathcal{P}^{\prime}$ e obtemos uma solução aproximada $y$ de $t^{\prime}(x)$. Finalmente, transformamos $y$ em uma solução do problema original.

O comportamento distinto com relação à aproximabilidade induz a definição de várias reduções e medidas de aproximação [Kan92, Sal96] objetivando respeitar as características diversificadas dos problemas e capturar a complexidade envolvida. Neste texto, utilizamos principalmente os seguintes conceitos de medida de aproximação e redução.

Definição 2.3.2. Seja $\mathcal{P}$ um problema NPO. Dada uma instância $x$ e uma solução viável $y$ de $x$, definimos a razão de aproximação de y com relação a $x$ como

$$
R(x, y)=\max \left\{\frac{m(x, y)}{o p t(x)}, \frac{o p t(x)}{m(x, y)}\right\} .
$$

Note que a razão de aproximação é sempre um número maior do que ou igual a 1 e está próxima de 1 quando o valor da solução viável está próxima do valor ótimo.

$\mathrm{Na}$ literatura, a medida de aproximação especificada na Definição 2.3 .2 é também denominada razão de desempenho (performance ratio) ou limite da razão (ratio bound).

Definição 2.3.3. Sejam $\mathcal{P}$ um problema em NPO, $A$ um algoritmo para $\mathcal{P}, A(x)$ a solução devolvida por $A$ quando aplicado a uma instância $x$ de $P$, e $r$ uma funçấo do tamanho das instâncias de $\mathcal{P}$ que assume somente valores positivos (eventualmente $r$ pode ser uma constante). Se para qualquer instância $x$ de tamanho $n, R(x, A(x)) \leq r(n)$, então dizemos que $\mathcal{P}$ tem uma $r(n)$-aproximação ou pode ser aproximado dentro de $r(n)$. Neste caso, também dizemos que $A$ é uma $r(n)$ aproximação ou um algoritmo $r(n)$-aproximado.

Não ocorrendo qualquer menção em contrário, referências à aproximabilidade de um problema NPO serão relativas às definições 2.3 .2 e 2.3.3. Também res- 
saltamos que $r(n)$ é uma razão de aproximação absoluta, porém omitiremos essa especificação, deixando-a subentendida.

Definição 2.3.4. Um algoritmo $A$ para $\mathcal{P}$ um problema NPO é chamado um PTAS, esquema de aproximação polinomial (FPTAS, esquema de aproximação completamente polinomial) se, para qualquer instância $x$ de $P$ e para qualquer racional $r>1, A(x, r)$ devolve uma solução viável cuja razão de aproximação é no máximo $r$ em tempo limitado por $q_{r}(|x|)\left(q_{r}\left(\frac{1}{r-1},|x|\right)\right)$, onde $q_{r}$ é um polinômio.

Estudos quanto à aproximabilidade de problemas NPO deram origem às seguintes classes de problemas: APX, para a classe de problemas que admitem uma solução $r$-aproximada; PTAS, para aqueles que possuem um esquema de aproximação polinomial; FPTAS referente aos problemas com esquema de aproximação completamente polinomial

Definição 2.3.5. O limiar de aproximação de um problema $\mathcal{P}$ em APX é um número real $r \geq 1$ tal que, para qualquer $\alpha>0, \mathcal{P}$ admite um algoritmo $(r+\alpha)$ aproximado mas $\mathcal{P}$ não admite um algoritmo $(r-\alpha)$-aproximado.

Definição 2.3.6 ([CKST99]). Sejam $\mathcal{P}$ e $\mathcal{P}^{\prime}$ dois problemas de otimização e $\alpha$ uma constante positiva. $\mathcal{P}$ é dito $\alpha-A P$ redutivel a $\mathcal{P}^{\prime}$, em simbolos $\mathcal{P} \leq_{A P}^{\alpha} \mathcal{P}^{\prime}$, se existem duas funções $f$ e $g$ tais que:

1. Para qualquer $x \in I_{\mathcal{P}}$ e para qualquer $r>1, f(x, r) \in I_{\mathcal{P}^{\prime}}$.

2. Para qualquer $x \in I_{\mathcal{P}}$, para qualquer $r>1$, e para qualquer $y \in \operatorname{sol}_{\mathcal{P}^{\prime}}(f(x, r))$, temos que $g(x, y, r) \in \operatorname{sol}_{\mathcal{P}}(x)$.

3. $f$ e $g$ são computáveis por dois algoritmos $T_{f}$ e $T_{g}$, respectivamente, cujos tempos de execução são polinomiais para qualquer $r$ fixo.

4. Para qualquer $x \in I_{\mathcal{P}}$, para qualquer $r>1$, e para qualquer $y \in \operatorname{sol}_{\mathcal{P}^{\prime}}(f(x, r))$,

$$
R_{\mathcal{P}^{\prime}}(f(x, r), y) \leq r \Rightarrow R_{\mathcal{P}}(x, g(x, y, r)) \leq 1+\alpha(r-1) .
$$

A tripla $(f, g, \alpha)$ é chamada uma AP-redução de $\mathcal{P}$ para $\mathcal{P}^{\prime}$. Observe que se $\mathcal{P} \leq_{A P}^{\alpha} \mathcal{P}^{\prime}$ para qualquer $\alpha>1$, então o limiar de aproximação de $\mathcal{P}$ é no máximo igual ao limiar de aproximação de $\mathcal{P}^{\prime}$. Em particular, se $\mathcal{P} \equiv_{A P}^{\alpha} \mathcal{P}^{\prime}$ para qualquer $\alpha>1$, então os dois problemas têm o mesmo limiar de aproximação.

O próximo teorema mostra que AP-redução preserva a existência de um esquema de aproximação polinomial. 
Teorema 2.3.1. Se $\mathcal{P}$ é um problema NPO, $\mathcal{P}^{\prime} \in \mathrm{PTAS}$ e existe uma AP-redução de $\mathcal{P}$ para $\mathcal{P}^{\prime}$, então $\mathcal{P} \in$ PTAS.

Uma questão natural a considerar quando tratamos de vários algoritmos de aproximação para um problema é determinar seu limite de aproximabilidade. Em outras palavras, até onde podemos promover melhorias em termos das razões de aproximação (limites de erro) e tempos de execução. A Seção 2.4 aborda justamente a qualidade de aproximação de problemas NPO.

\subsection{Qualidade de Aproximação}

Investigar a aproximabilidade de problemas de otimização NP via identificação de propriedades que os caracterizam é um tópico de pesquisa que tem merecido bastante atenção na última década. Ainda assim, existem inúmeras questões em aberto sobre esse assunto. A abordagem algorítmica, que se caracteriza pela busca de algoritmos ou esquemas de aproximação, bem como resultados sobre inaproximabilidade, também têm recebido muita atenção e têm igual relevância. Nesta direção, quando se obtém um resultado negativo sobre a existência de esquemas de aproximação (PTAS ou FPTAS), busca-se o melhor algoritmo de aproximação possível para o problema em questão.

Dizemos que um algoritmo de $r$-aproximação é o melhor possível para um dado problema se o problema de se obter uma $(r-\alpha)$-aproximação é NP-difícil para qualquer $\alpha>0$.

Ao discorrer sobre qualidade de aproximação [Hoc97] é oportuno destacar que esta tem estreita relação com a forma de apresentar o problema ou com a medida de aproximação usada. Como exemplo imediato mencionamos os problemas Max Conjunto Independente e Min Cobertura de Vértices (veja Seção 2.3), cujas soluções ótimas são dedutíveis uma da outra, mas suas soluções aproximadas são de qualidades bem diversas (quanto à razão de aproximação).

Outro exemplo interessante é o problema Max Mochila. Se considerarmos como medida de aproximação o erro absoluto, temos que não existe nenhum algoritmo com aproximação constante. Contudo, usando o erro relativo, obtemos um esquema de aproximação de tempo completamente polinomial (FPTAS). Veja a seguir a definição dos conceitos de erro absoluto e erro relativo. 
Definição 2.4.1. Seja $\mathcal{P}$ um problema NPO. Dada uma instância $x \in I_{\mathcal{P}}$ e uma solução $y \in \operatorname{sol}_{\mathcal{P}}(x)$, o erro absoluto de y com relação a opt $t_{\mathcal{P}}(x)$ é definido como

$$
\varepsilon_{\mathcal{P}}^{a}=\left|o p t_{\mathcal{P}}(x)-m_{\mathcal{P}}(x, y)\right|
$$

Definição 2.4.2. Seja $\mathcal{P}$ um problema NPO. Dada uma instância $x \in I_{\mathcal{P}}$ e uma solução $y \in \operatorname{sol}_{\mathcal{P}}(x)$, o erro relativo de y com relação a opt $(x)$ é definido como

$$
\varepsilon_{\mathcal{P}}^{r}(x, y)=\frac{\left|\operatorname{opt}_{\mathcal{P}}(x)-m_{\mathcal{P}}(x, y)\right|}{o p t_{\mathcal{P}}(x)}
$$

Dado um algoritmo de aproximação para um problema $P$, uma questão de interesse é saber se a aproximação obtida é a melhor possível para $P$ ou pode ser melhorada.

Por exemplo, para o problema Min Cobertura de Vértices, inicialmente uma aproximação logarítmica foi obtida e posteriormente foi melhorada para a constante 2. Conjectura-se que é impossível conseguir uma aproximação melhor. E por outro lado, sabe-se que aproximar este problema dentro de 1.1666 é NP-difícil [Hås01]. Encontra-se então em aberto o intervalo entre 2 e 1.1666, no sentido de que não podemos afirmar se aproximações melhores existem ou simplesmente falhamos em descobri-las até o momento. Outro exemplo é Max Corte. Neste caso, as razões de aproximação progrediram de 2 para 1.13823 [GW94].

Esquemas de aproximação polinomial (PTAS) são superiores às aproximações constantes: com eles é possível aproximar-se da razão 1 (isto é, da solução ótima) tanto quanto se queira. Neste caso, para cada $\epsilon>0$ fixo, o tempo de execução é polinomial no tamanho da entrada e $1+\epsilon$ é a razão de aproximação desejada.

Os esquemas não são precisamente o melhor resultado em aproximação porque o tempo de execução destes algoritmos pode ser exponencial em $\frac{1}{\epsilon}$, isto é, pode ser do tipo $2^{1 / \epsilon} p(|x|)$ ou $|x|^{1 / \epsilon}$, onde $|x|$ é o tamanho da entrada e $p$ é um polinômio (para $\epsilon$ fixo tais funções são polinomiais em $|x|$ ). O melhor resultado para um problema de otimização NP-difícil é um esquema de aproximação completamente polinomial (FPTAS), onde o tempo de execução é agora polinomial também em $\frac{1}{\epsilon}$.

Infelizmente, para muitos problemas não existe um FPTAS, a menos que $\mathrm{P}=\mathrm{NP}$. Isto ocorre para problemas fortemente NP-completos. Este conceito, apresentado em [GJ79], é para relacionar a causa da dificuldade de um problema apenas ao tamanho (ocorrência dos maiores valores na entrada) do mesmo. 
Definição 2.4.3. Dado um problema $\mathcal{P}$, e uma instância $x \in I_{\mathcal{P}}$ denotamos por maxint $_{\mathcal{P}}(x)$ o maior inteiro que ocorre em $x$, ou 0 se nenhum inteiro existe em $x$.

Definição 2.4.4. Um problema $\mathcal{P} \in \mathrm{NP}$ é NP-completo no sentido forte (ou fortemente NP-completo) se existe um polinômio $p: \mathbb{N} \rightarrow \mathbb{N}$ para o qual o problema, quando restrito a instâncias $x$ que satisfazem

$$
\operatorname{maxint}_{\mathcal{P}}(x) \leq p(|x|)
$$

é NP-completo.

Definição 2.4.5. Dado $\mathcal{P}$, um problema NP, dizemos que um algoritmo $A$ para $\mathcal{P}$ é pseudo-polinomial se, para qualquer instância $x \in I_{\mathcal{P}}$, A pode ser executado em tempo limitado por um polinômio em $|x|$ e $\operatorname{maxint}_{\mathcal{P}}(x)$.

Garey e Johnson mostraram que não existe esquema de aproximação completamente polinomial para um problema cuja versão de decisão é fortemente NPcompleta, a menos que $\mathrm{P}=\mathrm{NP}$. Com poucas exceções, uma notável delas sendo Max Mochila, muitos problemas são fortemente NP-completos. Max Mochila possui um FPTAS.

Ao considerarmos a razão de aproximação de um algoritmo, é importante distingüir entre razões absolutas e assintóticas, pois estas podem conduzir a resultados de complexidade contrastantes. É importante ater-se a essa distinção, pois em alguns casos a impressão que se tem é a de que a existência de certos algoritmos de aproximação parece violar alguns resultados de impossibilidade (com a qualificação "a menos que $\mathrm{P}=\mathrm{NP}$ ").

Apesar de não apresentarmos resultados a respeito de razões assintóticas, apresentamos a definição desse conceito ${ }^{2}$ para um melhor entendimento do comentário que efetuamos em seguida.

Definição 2.4.6. A razão de aproximação assintótica de um algoritmo de aproximação A para um problema $\mathcal{P}$ é definida como

$$
R_{A}^{\infty}=\inf \left\{r \geq 1 \mid \exists n \in Z^{+}, R(x, y) \leq r \forall x \in I_{\mathcal{P}} \text { tal que }|x| \geq n \text { e } y \in \operatorname{sol}_{\mathcal{P}}(x)\right\},
$$

onde $R(x, y)$ é como especificado na Definição 2.3.2.

\footnotetext{
${ }^{2}$ Esquemas de aproximação assintóticos são definidos analogamente.
} 
Um exemplo onde o contraste entre a razão absoluta e assintótica ocorre é no problema Min Empacotamento (Bin Packing), cuja versão de decisão é fortemente NP-completa e por conseguinte não possui FPTAS, a menos que P $=$ NP. Contudo, Karp e Karmarkar [KK82] obtiveram um esquema de aproximação assintótico completamente polinomial que devolve uma solução para uma instância $x$ que é no máximo $(1+\epsilon) \operatorname{opt}(x)+O\left(\left(\frac{1}{\epsilon}\right)^{6}\right)$.

Não sendo suficiente todas estas questões intrigantes, surgiu mais uma perspectiva para os problemas de otimização NP. Estudar as propriedades de aproximabilidade da versão com pesos dos problemas de otimização (generalizando a versão sem pesos, ou equivalentemente, de peso constante 1). A Seção 2.5 discute a aproximabilidade destes problemas e as relações entre as versões com e sem pesos de um problema NPO.

\subsection{Problemas com Pesos}

Alguns problemas apresentam complexidade distinta quando somente pesos positivos são usados e quando pesos arbitrários (não necessariamente polinomialmente limitados) são permitidos.

Muitos algoritmos de aproximação para problemas de otimização têm garantia de uma certa razão de aproximação somente se pesos não são permitidos. Para conseguir o mesmo desempenho no caso com pesos, tem-se que considerar técnicas diferentes e freqüentemente mais complicadas. Por exemplo, o algoritmo 2-aproximado para a versão sem pesos de Min Cobertura de Vértices é baseado em uma simples estratégia "gulosa" para encontrar um emparelhamento máximo no grafo. No entanto, para se obter o primeiro algoritmo 2-aproximado para a versão com pesos, em vez disto, uma formulação do problema baseada em programação linear foi usada. Em alguns casos, não é conhecido se a mesma razão de aproximação pode ser obtida. Por exemplo, enquanto a versão sem pesos de Max Clique é aproximável dentro de $O\left(n / \log ^{2} n\right)$, onde $n$ denota o número de vértices no grafo [BH92], o melhor algoritmo de aproximação para a versão com pesos deste problema atinge um fator de $O\left((\log \log n)^{2} n / \log ^{2} n\right)$ [Hal95].

É bem conhecido que muitos problemas de otimização NP-difíceis tornamse tratáveis se um limite polinomial é imposto sobre os pesos que aparecem na instância. Por exemplo, o problema Min Partição pode ser resolvido em tempo 
$O(n b)$ onde $n$ denota o número de elementos e $b$ denota a soma dos pesos destes elementos: claramente, se qualquer limite superior fosse colocado sobre os pesos, mesmo até uma função polinomial em $n$, este algoritmo seria um algoritmo polinomial para o problema restrito. Crescenzi et al. [CST01] estudaram a complexidade relativa às propriedades de aproximação de três possíveis versões de um problema de otimização: aquela com pesos arbitrários, com pesos polinomialmente limitados e sem pesos. As considerações efetuadas nesta seção podem sugerir que as três versões têm graus de dificuldade de aproximação diferentes. Contudo, foi provado que para muitos problemas interessantes como Min Cobertura de Vértices, Min Sat, Max Corte, Max 2Sat e Max Exact $k$ Sat o limiar de aproximação (Definição 2.3.5) é exatamente o mesmo para as três versões. (No Apêndice desta tese definimos todos os problemas aqui mencionados.)

Definição 2.5.1. Um problema de otimização $\mathcal{P}$ é um problema-subconjunto se:

- Qualquer instância $x$ de $\mathcal{P}$ é uma tripla $\left(U, x^{\prime}, w\right)$, onde $U=\left\{u_{1}, \ldots, u_{n}\right\}$ é um conjunto de objetos, $x^{\prime}$ é uma cadeia (possivelmente vazia), e $w: U \rightarrow \mathbb{N}$ atribui um peso $w(u)$ para qualquer objeto $u \in U$.

- A qualquer solução $y \in \operatorname{sol}(x)$ está associado um subconjunto $S \subseteq U$, e a medida de y é igual a $\sum_{u \in S} w(u)$.

Por exemplo, considere a versão com pesos de Max Sat. Uma instância é uma tripla $(C, \epsilon, w)$ onde $C$ é o conjunto de cláusulas e $w: C \rightarrow \mathbb{N}$ é a função que atribui pesos às cláusulas. Uma solução é uma atribuição de valores 'verdadeiro' ou 'falso' para as variáveis em $C$ : a qualquer solução podemos então associar o subconjunto $C^{\prime} \subseteq C$ de cláusulas satisfeitas e a medida desta atribuição é a soma dos pesos dos elementos de $C^{\prime}$.

Definição 2.5.2. Um problema-subconjunto $\mathcal{P}$ é refinado se, para qualquer instância $\left(U, x^{\prime}, w\right)$, se y é uma solução viável então para qualquer função $w^{\prime}$ : $U \rightarrow \mathbb{N}$, y é também uma solução para $\left(U, x^{\prime}, w^{\prime}\right)$. Em outras palavras, esta propriedade diz que a definição de uma solução viável de $\mathcal{P}$ é independente dos pesos.

É sabido que Papadimitriou e Yannakakis [PY91] definiram e estudaram as classes MAX SNP e MAX NP, ambas contendo problemas APX e derivadas da caracterização lógica de NP apresentada por Fagin [Fag74]. Contudo, neste mesmo 
trabalho também foram exibidas variações destas classes com pesos positivos. Esses autores provaram que os problemas destas classes mantêm suas propriedades de aproximação. Posteriormente, Zimand [Zim99] estudou as propriedades de aproximação de problemas com pesos definidos logicamente, permitindo pesos negativos (mas funções objetivo não-negativas). Concluiu que a dificuldade de aproximação cresce drasticamente quando pesos negativos são permitidos. Isto é um contraste com o bom comportamento dos problemas das classes MAX SNP e weight(+)-MAX SNP, relativas respectivamente àqueles sem pesos e com pesos apenas positivos associados aos dados de entrada, que são comprovadamente aproximáveis dentro de uma constante [PY91]. Este fato indica a necessidade de investigação dos motivos que dão origem a mais esta anomalia no comportamento de problemas de otimização com relação à aproximação.

Crescenzi e Trevisan [CT00] também mostraram que qualquer problema aproximável com pesos arbitrários é redutível para um problema aproximável com pesos polinomialmente limitados. Khuller, no capítulo 6 de [Hoc97], discute as versões com e sem pesos dos problemas de conectividade de arestas, conectividade de vértices, conectividade forte e aumento de conectividade.

Antes de descrever nossos resultados, apresentamos resumidamente o estado-daarte na área de aproximabilidade de problemas NPO. Nosso objetivo é comentar brevemente e em ordem cronológica alguns resultados ou fatos que foram marcantes na área de aproximação.

\subsection{Estado-da-arte}

\subsubsection{3-1986: Resultados Dispersos}

Algoritmos de aproximação surgiram na área de Pesquisa Operacional, ainda que não com esta terminologia. Um dos trabalhos pioneiros nesta área foi apresentado em 1966 por Graham [Gra66], para um problema de escalonamento de tarefas em máquinas. As definições formais de razão e algoritmo de aproximação foram exibidas primeiramente por Johnson [Joh74].

Do ponto de vista algorítmico, houve um desenvolvimento rápido em poucos anos. Contudo, sobre inaproximabilidade, a única alternativa era relacionar as propriedades de aproximação com a teoria da NP-completude. 
Uma tentativa bem sucedida foi a conexão estabelecida por Garey e Johnson [GJ78] entre a classe FPTAS e o conceito de NP-completude no sentido forte. Provou-se que quando um problema é fortemente NP-difícil então este não pertence à classe FPTAS, a menos que $\mathrm{P}=\mathrm{NP}$. Em conseqüência, provas dessa natureza tornaram-se freqüentes em artigos sobre aproximabilidade de problemas de otimização.

O próximo objetivo foi descobrir propriedades que pudessem ser usadas para provar a não-pertinência à classe PTAS. Uma observação simples é a seguinte. Suponha que para alguma constante $k$, dada uma instância $x$ de um problema de otimização $A$, é NP-difícil dizer se o ótimo é maior do que $k$ ou não. Um algoritmo $r$-aproximado, onde $r<(k+1) / k$, pode ser usado para responder tal questão e $\operatorname{assim} A$ não pode pertencer à classe PTAS. Alguns resultados podem ser obtidos com esta técnica, porém para alguns problemas interessantes como Max Clique, Min Cobertura de Vértices e Max 3Sat tal abordagem é ineficiente, visto que para um $k$ fixo é possível responder em tempo polinomial se o ótimo é maior do que $k$ ou não.

Posteriormente, surgiu a técnica gap, usada por Sahni e Gonzalez [SG76] para mostrar que Min Caixeiro Viajante não pertence à classe APX. Esta técnica também é aplicável somente para poucos casos, devido à dificuldade em se precisar o gap necessário para cada problema.

Outra técnica, surgida na década de 70, foi a utilização de grafos produto. Com esta técnica Garey e Johnson [GJ76] mostraram que Min Coloração não pode ser $r$-aproximado para $r<2$. Tal técnica foi também utilizada para mostrar a autoimprovabilidade de Max Clique [GJ79, BS92]. Esta propriedade implica que Max Clique está em PTAS ou não está em APX.

\subsubsection{7-1990: Classes de Problemas}

Inspecionando-se os resultados anteriores sobre inaproximabilidade, observa-se que quase todos tratam da complexidade do problema de decisão associado ao problema de otimização de interesse. Um momento importante no contexto da teoria de aproximação foi a percepção de que uma abordagem diferente tinha que ser adotada: movendo-se das linguagens associadas para os problemas de otimização em si. Em outras palavras, classes de problemas de otimização precisavam ser definidas, 
de modo que a partir delas fosse possível provar resultados de completude ou de dificuldade para estas classes, utilizando-se reduções que preservam aproximação.

A idéia de definir noções de redução, diretamente sobre problemas de otimização, tinha sido já concebida nos primeiros anos da década de 80 nos artigos de Paz e Moran [PM81] e Ausiello, D'Atri e Protasi [ADP80]. Contudo, nestes trabalhos resultados de completude em classes de problemas de otimização não foram obtidos.

Krentel [Kre88] foi o primeiro a exibir resultados de completude. Apesar de neste artigo não ser definida uma redução que preserva aproximação, pois o foco de sua pesquisa era a dificuldade de computar soluções ótimas, surpreendentemente foi mostrado que um problema em FPTAS (a saber, Max Mochila) é mais difícil do que Max Clique (mais recentemente, tem sido mostrado que Max Clique é muito difícil de ser aproximado). Um caminho para interpretar este resultado é que a dificuldade de resolver exatamente um problema de otimização não está relacionada com a dificuldade de sua versão aproximada.

Orponen e Manilla [OM87] mostraram pela primeira vez que resultados de completude em classes de aproximação (a saber, MIN NPO), via reduções que preservam aproximação, implicam resultados de inaproximabilidade. Crescenzi e Panconesi [CP91] estenderam esta idéia para outras classes de aproximação como MAX NPO e APX.

Posteriormente, outros resultados de completude foram obtidos por Berman, Schnitger [BS92] e Kann [Kan94] em classes de problemas com função objetivo polinomialmente limitadas.

Papadimitriou e Yannakakis [PY91] desenvolveram o trabalho de maior repercussão deste período, onde uma abordagem radicalmente nova foi adotada. Em vez de procurar por problemas completos em classes de aproximação computacionalmente definidas, eles focaram na caracterização lógica de NP, devida a Fagin [Fag74], e definiram uma classe de problemas de otimização APX chamada MAX NP. Muitos problemas foram classificados, mas não foram obtidos resultados de completude. Uma restrição na definição de MAX NP produziu uma subclasse denominada MAX SNP, a qual contém problemas interessantes como Max Corte e Max 3Sat. Para esta classe, resultados de completude foram extraídos, bem como, mostrou-se que os problemas MAX SNP-difíceis não possuem PTAS.

Esta abordagem sintática foi amplamente explorada em uma série de artigos. 
Panconesi e Ranjan [PR93] mostraram que Max Clique não está em MAX SNP e definiram uma classe onde Max Clique é completo. Kolaitis e Thakur efetuaram uma ampla investigação em classes sintáticas [KT94] e estenderam a abordagem para problemas de minimização [KT95]. Outros pesquisadores exploraram a definibilidade lógica considerando pontos fixos [BCG93].

\subsubsection{1-1993: A Conexão PCP}

A visão sintática da aproximabilidade produziu muitos resultados relacionando a dificuldade dos problemas entre si, mas não com as noções clássicas da teoria da complexidade. A conexão com paradigmas computacionais conhecidos ainda estava por vir e eventualmente ocorreu em uma das mais inesperadas direções: provas verificáveis probabilisticamente.

A idéia de estender o modelo de verificação determinística de provas, permitindo aleatoriedade e interação foi apresentada independente e simultaneamente por Babai [Bab85, BM88] e por Goldwasser, Micali e Rackoff [GMR89]. A classe de linguagens de tais sistemas interativos de provas (probabilísticos) foi chamada de IP.

Ben-Or, Goldwasser, Kilian e Wigderson [BOGKW90] consideraram uma extensão do modelo, onde o verificador interage com dois (ou mais) provadores (que não se comunicam uns com os outros). As linguagens admitindo tais sistemas de provas formam a classe MIP. Fortnow, Rompel e Sipser [FRS94] apresentaram uma caracterização alternativa de MIP: uma linguagem MIP admite provas (exponencialmente longas) que podem ser verificadas em tempo polinomial por um verificador probabilístico.

Inicialmente, os modelos mencionados foram considerados apenas um pouco mais potentes do que NP, isto até Lund, Fortnow, Karloff e Nisan [LFKN92] provarem que IP contém a hierarquia de tempo polinomial. Logo depois, Shamir [Sha92] mostrou que IP é igual a PSPACE e Babai, Fortnow e Lund [BFL91] provaram que MIP é igual a NEXP. Este último resultado foi particularmente surpreendente: significa que, para qualquer linguagem admitindo provas de pertinência exponencialmente longas, tais provas podem ser verificadas em tempo polinomial com baixa probabilidade de erro.

Logicamente, a próxima etapa foi provar que qualquer linguagem NP tem provas 
de pertinência verificáveis em tempo polilogarítmico. Isto foi mostrado por Babai, Fortnow, Levin e Szegedy [BFLS91]. Quase simultaneamente, Feige, Goldwasser, Lovász, Safra e Szegedy [FGL $\left.{ }^{+} 96\right]$ exibiram um sistema de verificação de prova melhorado para NP e propuseram a redução que revolucionou a teoria da aproximação. A saber, eles mostraram como codificar a computação de um verificador em um grafo, de tal forma que existe uma correspondência entre cliques no grafo e provas de supostas pertinência, e entre o tamanho do clique e a probabilidade da suposta prova ser aceita. Assim, um algoritmo de aproximação para Max Clique pode ser usado para aproximar a probabilidade de aceitação do verificador, e um gap entre a probabilidade de aceitação de provas corretas e erradas traduz-se em resultado de inaproximabilidade para Max Clique.

Arora e Safra [AS98] observaram que para obter melhorias nos resultados discutidos acima, não seria necessário limitar o tempo de execução do verificador mas somente o número de bits aleatórios e o número de bits de consulta usados pelo verificador. Surge então a notação $\operatorname{PCP}(r(n), q(n))$ que denota a classe de linguagens possuindo provas de pertinência, para elementos de comprimento $n$, que podem ser verificadas probabilisticamente (Probabilistically Checkable Proofs) usando $O(r(n)$ ) bits aleatórios e $O(q(n))$ bits de consulta.

O próximo passo foi a percepção de que a dificuldade de aproximação de outros problemas podia ser estabelecida usando a conexão PCP. Arora, Lund, Motwani, Sudan e Szegedy $\left[\mathrm{ALM}^{+} 98\right]$ provaram que NP $=\mathrm{PCP}(\log n, 1)$, o conhecido Teorema PCP, e mostraram que problemas MAX SNP-difíceis não possuem PTAS.

Além desta aplicação para problemas MAX SNP-difíceis, o Teorema PCP também implica que é NP-difícil aproximar Max Clique dentro de $n^{c}$, para algum $c>0$, onde $n$ é o tamanho da instância sendo considerada.

A nova caracterização de NP, pode ser informalmente enunciada como segue. Suponha que queiramos certificar a resposta 'SIM' para uma instância de tamanho $n$ de um dado problema de decisão. Então existe uma prova $\Pi$ verificável probabilisticamente que, ao ser examinada, apenas uma quantidade não maior do que uma constante independente de $n$ de caracteres é lida, sendo a escolha destes caracteres baseada em apenas $O(\log n)$ bits aleatórios.

Consideremos o seguinte exemplo concreto: decidir se um certo grafo $G$ é hamiltoniano. Suponha que um dado grafo $G$ admite uma solução, isto é, tem um circuito hamiltoniano. Então é possível apresentar uma prova $\Pi$ deste fato com a 
seguinte propriedade: lê-se uma quantidade pequena de caracteres desta prova e escolhe-se a localização destes caracteres através de um número também pequeno de bits aleatórios. Genericamente, se $G$ tivesse $n$ vértices, um número constante de caracteres desta prova seriam examinados e seriam necessários $O(\log n)$ bits aleatórios para a escolha destes caracteres. Note que, em particular, muito pouca informação seria adquirida neste processo: a leitura de uma quantidade constante de bits da prova não pode, por exemplo, fornecer muita informação sobre como é a solução do problema. Entretanto, esta leitura será suficiente para convencer que uma solução existe.

Formalmente, podemos exibir a seguinte descrição do resultado em questão. Sejam $\sum=\{0,1\}$ e $L \subset \sum^{*}$ um problema NP, isto é, uma linguagem cujos elementos $x \in L$ admitem certificados de pertinência $y_{x}$ simples e curtos. Isto significa que para provarmos que $x \in L$, basta apresentarmos este certificado $y_{x}$ como uma demonstração deste fato. Sendo simples e curta, esta prova pode ser eficientemente verificada em tempo polinomial.

No sistema de provas PCP, o verificador $V$ é uma máquina de Turing determinística de complexidade de tempo polinomial que recebe como entrada uma palavra $x \in \sum^{*}$ e que tem acesso a uma seqüência de bits aleatórios $\sigma \in \sum^{*}$. Este verificador também tem acesso a uma palavra $\Pi=\pi_{i_{1}} \pi_{i_{2}} \ldots \pi_{i_{|\Pi|}} \in \sum^{*}$ através de um oráculo. Uma vez dadas a entrada $x$ e a seqüência de bits aleatórios $\sigma$, o verificador $V$ sabe quais caracteres $\pi_{i}$ de $\Pi$ vai querer acessar durante a sua computação. Em conseqüência, dados $x$ e $\sigma$, o verificador $V$ pode pedir ao oráculo, de uma só vez, todos os caracteres $\pi_{i_{1}} \ldots \pi_{i_{l}}$ de que $V$ necessitará, não sendo o valor destes caracteres levado em conta na decisão de quais caracteres serão lidos. Por causa disto, dizemos algumas vezes que o verificador $V$ é não-adaptativo. No final da sua computação, a saída, denotada por $V(x, \sigma, \Pi)$, será 'ACEITA' ou 'REJEITA'.

Sejam $r(n)$ e $q(n)$ duas funções reais definidas sobre os inteiros não negativos $n$. Um verificador $V$ é dito $(r(n), q(n))$-restrito se existem duas funções inteiras $\hat{\mathrm{r}}(n)=$ $O(r(n))$ e $\hat{\mathrm{q}}(n)=O(q(n))$ tais que $V$, com qualquer entrada $x$ de comprimento $|x|=n$ e qualquer seqüência de bits aleatórios $\sigma$, faz uso em sua computação de não mais do que os primeiros $\hat{\mathrm{r}}(n)$ bits de $\sigma$, e pede ao oráculo não mais que $\hat{\mathrm{q}}(n)$ caracteres $\pi_{i}$ de $\Pi$. No que segue, escrevemos $P_{\sigma}$, para denotar a probabilidade com relação à seqüência de bits aleatórios $\sigma$. Formalmente, embora os verificadores usem apenas um segmento inicial finito de $\sigma$, podemos assumir que $\sigma$ tem comprimento infinito. 
A linguagem $L \subset \sum^{*}$ está em $\operatorname{PCP}(r(n), q(n))$ se e só se existe um verificador $(r(n), q(n))$-restrito $V$ para o qual valem as seguintes duas asserções.

- Para todo $x \in L$, existe uma prova $\Pi=\Pi_{x} \in \sum^{*}$ tal que

$$
P_{\sigma}\{V(x, \sigma, \Pi)=A C E I T A\}=1 \text {. }
$$

- Para todo $x \notin L$ e para todo $\Pi \in \sum^{*}$, temos

$$
P_{\sigma}\{V(x, \sigma, \Pi)=A C E I T A\}<\frac{1}{4} .
$$

Outros resultados relacionados com verificação de provas foram: os limites inferiores de $\frac{1}{4} \log n$ para a aproximabilidade de Min Cobertura de Conjuntos e o de $n^{c}$ para Min Coloração para algum $c>0$ [LY94]. As reduções usadas não são do verificador do Teorema PCP, mas de uma variação apresentada em [LS97, FL92]. Bellare [Bel93] foi talvez o primeiro a explorar a conexão entre tais sistemas de prova e problemas de aproximação.

\subsubsection{Resultados Recentes}

Devido ao rápido e crescente desenvolvimento da teoria da aproximação nos últimos anos, podemos afirmar que muitas resenhas recentes já se encontram ligeiramente desatualizadas. Ultimamente vários resultados de inaproximabilidade têm sido obtidos. Estes são alcançados usando versões cada vez mais refinadas do Teorema PCP e descobrindo melhores reduções.

Recentemente, resultados de inaproximabilidade justos têm sido estabelecidos para muitos problemas de otimização. Por resultados justos entendamos que: inicialmente, a inaproximabilidade era mostrada garantindo a existência de constantes positivas que caracterizavam algum limite inferior para aproximabilidade; atualmente, é possível exibir-se efetivamente os valores específicos de tais constantes para cada problema estudado.

Ao abordarmos o impacto do Teorema $\mathrm{PCP}$ em resultados estruturais, podemos citar os seguintes resultados.

Khanna, Motwani, Sudan e Vazirani [KMSV99] usaram o Teorema PCP para mostrar que Max 3Sat é completo para APX PB (a restrição de APX para problemas com função objetivo polinomialmente limitada). A técnica de redução usada 
transforma muitos resultados de dificuldade em resultados de completude e definitivamente colapsa a abordagem baseada em definibilidade lógica com classes de complexidade computacionais. Infelizmente, isto é válido apenas no contexto de classes de aproximação polinomialmente limitadas.

Em uma linha de pesquisa diferente, Chang, Gasarch e Lund [Cha96, CGL97] usam o Teorema PCP para investigar a complexidade de consulta (query) de problemas de aproximação. Eles exibiram limites superiores e inferiores para o número de consultas NP que podem ser codificadas em um problema de aproximação. Em conseqüência, é estritamente mais fácil computar uma 2-aproximação do número clique de um grafo do que computar o número clique exatamente.

As seções históricas na monografia elaborada por Bellare, Goldreich e Sudan [BGS98] exibem completa e detalhadamente as melhorias que culminaram com os resultados recentes de inaproximabilidade.

Abordando as tendências atuais para o projeto de algoritmos eficientes de aproximação, citamos programação linear, aleatorização e programação semi-definida. No que segue, comentamos brevemente estas técnicas.

A compreensão matemática e algorítmica de programas lineares deram origem a muitas idéias e ferramentas ainda em uso atualmente no estudo de problemas de otimização.

O esquema geral utilizado para algoritmos de aproximação é:

1. Formular o problema como um programa linear inteiro (PLI).

2. Relaxar para um programa linear (PL).

3. Usar o PL (e sua solução) para obter uma solução para o PLI.

Existem várias maneiras diferentes de executar o passo 3 do esquema acima. Uma delas é o método guloso. O uso deste método para Min Cobertura de Conjuntos gera um algoritmo $H_{g}$-aproximado, onde $H_{n}$ é o n-ésimo número harmônico e $g$ é o tamanho do maior conjunto da coleção de entrada do algoritmo.

Johnson e Lovász [Joh74, Lov75] foram os primeiros que demonstraram a aproximação mencionada para o caso sem pesos, onde a cada passo selecionase o conjunto que cobre o maior número possível de elementos não cobertos. Chvátal [Chv79] estendeu a aplicabilidade do método guloso para Min Cobertura 
de Conjuntos com pesos. Neste caso, a cada passo seleciona-se o conjunto que minimiza a razão entre o peso do conjunto sendo considerado e todos os elementos ainda não cobertos no referido conjunto (custo por elemento adicional coberto).

Um outro método é aquele de arredondamento, onde obtemos uma solução viável para o PLI original arredondando os valores das variáveis que não satisfazem as restrições de integridade. Utilizando-se este método para Min Cobertura de Conjuntos obtém-se um algoritmo $f$-aproximado, onde $f$ é o número máximo de conjuntos que contêm qualquer dado elemento.

Uma terceira maneira de se obter uma solução para o PLI é fazer o arredondamento da solução dual da relaxação. O uso deste método sobre o Min Cobertura de Conjuntos também fornece uma $f$-aproximação.

Outra alternativa é o método primal-dual, proposto por Dantzig, Ford, e Fulkerson [DFF56]. Esta abordagem no âmbito dos algoritmos de aproximação para problemas de otimização tem se fortalecido nos últimos anos como uma ferramenta relativamente genérica, em contraste com o procedimento usual de elaborar um algoritmo baseando-se na estrutura particular do problema. A força do método primal-dual ficou evidenciada por uma série de artigos desenvolvendo esta técnica para problemas de projeto de redes [AKR95, GW95a, WGMV95, RW02]. Quando aplicado ao Min Cobertura de Conjuntos, este método constrói uma $f$ aproximação.

Como referências para o estudo de algoritmos baseados em programação linear indicamos Hochbaum [Hoc97], Vazirani [Vaz00] e Williamson [Wil98]. Em geral, o uso de técnicas de relaxação de programas lineares inteiros, o estudo do seu dual e o uso de folgas complementares têm produzido importantes resultados para problemas de otimização NP.

Particularmente, o método da relaxação pode também ser combinado com programas semi-definidos, os quais podem ser resolvidos em tempo polinomial. Programação semi-definida é uma generalização de programação linear.

Goemans e Williamson [GW95b] apresentaram para muitos problemas NPdifíceis algoritmos de aproximação eficientes usando essa abordagem. Entre eles, mencionamos aquele desenvolvido para Max Corte com uma aproximação de 1.13823 .

Também mencionamos como tendência atual o desenvolvimento de algoritmos 
aleatórios que recebem como entrada não só os dados de uma instância do problema em questão, mas também uma seqüência de bits aleatórios. Baseados nestes dados, executam algumas computações e param. Mesmo para uma entrada fixa, diferentes execuções de um algoritmo aleatório podem gerar resultados diferentes. Por esta razão, uma descrição das propriedades de um algoritmo aleatório envolve afirmações probabilísticas. Por exemplo, o tempo de execução é uma variável aleatória, como também o valor da solução encontrada. Neste caso, quando estimamos o valor esperado da variável aleatória, consideramos o desempenho médio do algoritmo sobre todas as possíveis execuções.

No contexto de algoritmos de aproximação aleatórios é usado o conceito de razão de aproximação esperada. Usando aleatorização obtém-se para Max Sat uma 2-aproximação e um algoritmo $\frac{e}{e-1}$-aproximado (arredondamento aleatório). Utilizando uma abordagem ainda probabilística para escolher o melhor dos dois algoritmos aproximados mencionados, consegue-se uma $\frac{4}{3}$-aproximação. Para o problema Max Corte é possível obter um algoritmo 2-aproximado.

É possível transformar algoritmos aleatórios em determinísticos. Um dos métodos usados para desaleatorização é aquele das esperanças condicionais. Não definiremos aqui estes conceitos. Sugerimos que o leitor interessado consulte os textos $\left[\mathrm{ACG}^{+}\right.$99, Vaz00, Wil98].

Alguns trabalhos [Sze94, BS94, KG98] indicam como questão aberta os limites do uso da aleatorização no campo da aproximabilidade. Assim, o interesse é delimitar a potência de tal técnica não apenas para desenvolver algoritmos mas para provar resultados de não-aproximabilidade.

Resultados a respeito dos limites de desempenho de muitos problemas de otimização NP podem ser encontrados em [CK98], uma lista constantemente atualizada.

Após discorrer sobre a área de aproximabilidade de problemas de otimização NP, apresentar as definições básicas e abordar algumas questões de interesse, nos capítulos seguintes passamos a investigar, sob o ponto de vista algorítmico, vários problemas de particionamento de um grafo em subgrafos conexos. 


\title{
Bipartição Conexa Balanceada
}

\begin{abstract}
"A formulação de um problema é mais essencial do que sua solução, a qual pode ser simplesmente uma questão de destreza matemática ou experimental. Elaborar novas perguntas, novas possibilidades, e considerar problemas conhecidos sob um novo ponto de vista requer criatividade e marca avanços reais na ciência." (Albert Einstein)
\end{abstract}

Muitos algoritmos para problemas em grafos utilizam o seguinte procedimento recursivo: dado um grafo conexo $G$, particione $G$ em dois subgrafos conexos $G_{1}$ e $G_{2}$ de tamanhos aproximadamente iguais, resolva os correspondentes subproblemas para os grafos $G_{1}$ e $G_{2}$ e de suas soluções obtenha uma solução para o grafo original G. A exigência de que a partição seja tão balanceada quanto possível é natural, visto que minimiza a profundidade da recursão e a discrepância dos tamanhos dos grafos usados em qualquer nível do processo de recursão.

Estamos interessados em problemas dessa natureza, onde $G=(V, E)$ é um grafo conexo com pesos associados aos seus vértices e os subgrafos $G_{1}$ e $G_{2}$ são induzidos por uma partição $\left(V_{1}, V_{2}\right)$ de $V$. Neste caso, em vez de considerar as cardinalidades de $V_{1}$ e $V_{2}$, consideramos os pesos desses conjuntos. A definição formal desse problema, que será objeto de estudo deste capítulo, será dada a seguir.

Lembramos que, se $G=(V, E)$ é um grafo conexo, então dizemos que uma 
2-partição (ou bipartição) $\left(V_{1}, V_{2}\right)$ de $V$ é uma 2-partição (ou bipartição) conexa de $G$ se $G\left[V_{1}\right]$ e $G\left[V_{2}\right]$ são conexos. E para uma função (peso) $w: V \rightarrow Z_{+}$e um subconjunto $V^{\prime} \subseteq V$, denotamos por $w\left(V^{\prime}\right)$ a soma (dos pesos dos vértices em $V^{\prime}$, isto é,) $w\left(V^{\prime}\right)=\sum_{v \in V^{\prime}} w(v)$.

\section{Max 2-Partição Conexa Balanceada $\left(\mathrm{PCB}_{2}\right)$}

Instância: um grafo $G=(V, E)$ e uma função $w: V \rightarrow Z_{+}$.

Solução viável: uma 2-partição conexa $\left(V_{1}, V_{2}\right)$ de $G$.

Função: $\min \left\{w\left(V_{1}\right), w\left(V_{2}\right)\right\}$.

Objetivo: maximizar.

O caso especial do problema acima onde todos os vértices têm peso 1 será denominado de $\mathrm{PCB}_{2}$ sem pesos. Observamos que se os pesos de todos os vértices são uniformes (não necessariamente unitários), então temos um caso equivalente àquele em que todos os pesos são iguais a 1.

Como estamos interessados no estudo do $\mathrm{PCB}_{2}$ sob o ponto de vista algorítmico, é natural que este estudo comece pela identificação de sua complexidade computacional. Na próxima seção apresentamos resultados dessa natureza. A seguir, na Seção 3.2 mostramos resultados sobre a dificuldade de se obter soluções aproximadas para o $\mathrm{PCB}_{2}$. Finalmente, na Seção 3.3 apresentamos algoritmos de aproximação para o $\mathrm{PCB}_{2}$, bem como um esquema de aproximação para uma classe especial de grafos. Também discutimos aspectos quanto à aproximabilidade, comparando os casos com pesos e sem pesos.

\subsection{Complexidade Computacional}

Relatamos inicialmente os resultados já conhecidos na literatura para Max 2Partição Conexa Balanceada. Este problema, na sua versã̃o sem pesos, pode ser resolvido em tempo polinomial para as seguintes classes de grafos:

- grafos 2-conexos [Lov79, GMM97];

- grafos nos quais cada bloco tem no máximo dois pontos de articulação ${ }^{1}$ [Chl96, AC99];

\footnotetext{
${ }^{1}$ Este resultado inclui a classe de grafos com no máximo dois pontos de articulação, um resultado provado por Galbiati et al. em 1995 [GMM95, GMM97].
} 
- grafos nos quais cada bloco tem no máximo $p$ pontos de articulação conectados como um clique, onde $p$ é uma constante [AC99].

A prova deste último resultado nos permite concluir que o problema $\mathrm{PCB}_{2}$ sem pesos para esta classe de grafos pode ser resolvido em tempo polinomial se $p=O(\log n)$, onde $n$ é o número de vértices do grafo. Discutiremos mais adiante outros resultados relacionados a este.

Exibimos na Tabela 3.1 as classes de grafos para as quais foi provado ${ }^{2}$ que $\mathrm{PCB}_{2}$ sem pesos é NP-difícil.

\begin{tabular}{|c|c|}
\hline \hline CLASSE DE GRAFOS & REF. \\
\hline conexos & {$[\mathrm{CGM} 83]$} \\
\hline bipartidos & {$[\mathrm{DF} 85]$} \\
\hline com algum bloco $\operatorname{contendo} \Omega(\log n)$ pontos de articulação & {$[\mathrm{AC} 99]$} \\
\hline
\end{tabular}

Tabela 3.1: Classes de grafos para as quais $\mathrm{PCB}_{2}$ sem pesos é NP-difícil.

Mencionamos até agora apenas os resultados para a versão do $\mathrm{PCB}_{2}$ sem pesos. Quanto ao caso mais geral, com pesos quaisquer, temos que o $\mathrm{PCB}_{2}$ pode ser polinomialmente resolvido para escadas (ladders) [BLLS01] e claramente para árvores. Sabe-se também que este problema é NP-difícil para grafos planares e também para grades (com pelo menos 3 colunas) [BLLS98] (além dos casos mencionados na tabela acima).

Nesta seção relatamos os resultados que encontramos na literatura sobre o $\mathrm{PCB}_{2}$; na próxima seção apresentamos nossa contribuição sobre este problema ainda nesta linha de complexidade computacional.

\subsubsection{NP-completude}

Como o $\mathrm{PCB}_{2}$ sem pesos para grafos 2-conexos é um problema fácil, uma pergunta que surge naturalmente é se no caso geral, com pesos, isso também ocorre. Veremos que neste caso, o $\mathrm{PCB}_{2}$ é NP-difícil, no sentido forte.

Vamos a seguir provar que a versão de decisão do Max 2-Partição Conexa Balanceada restrito a grafos 2-conexos é um problema fortemente NP-completo. A prova

\footnotetext{
${ }^{2}$ Observamos que a prova para a terceira classe, devida a Alimonti e Calamoneri [AC99] necessita de um pequeno ajuste, mas está essencialmente correta.
} 


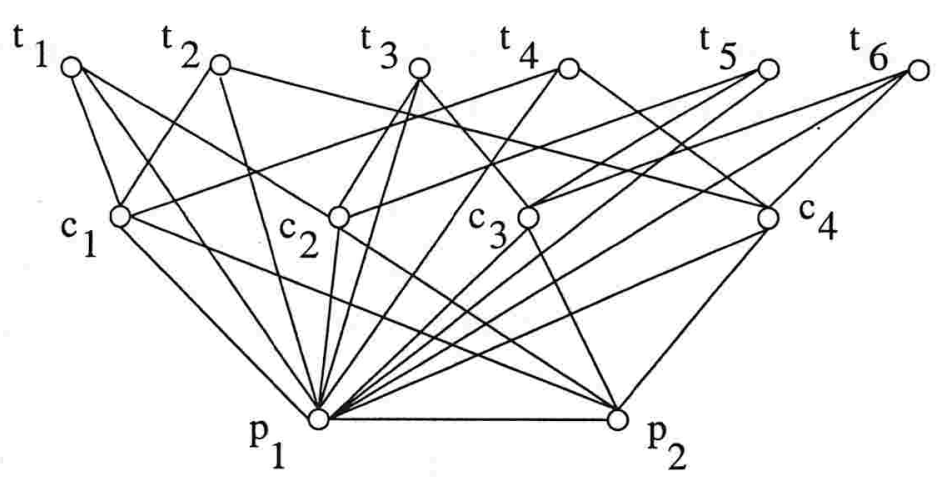

Figura 3.1: Exemplo de grafo gerado pela redução.

utiliza idéias semelhantes àquelas apresentadas por Galbiati et al. [CGM83], para grafos conexos e sem pesos. Observamos que o resultado apresentado por Simeone et al. [BLLS98] sobre a NP-completude do $\mathrm{PCB}_{2}$ restrito a grades (e portanto para grafos 2-conexos) não é no sentido forte.

Para este propósito, consideramos o problema Cobertura Exata por 3-Conjuntos (X3C) definido como segue: dada uma família $C=\left\{c_{1}, \ldots, c_{s}\right\}$ de subconjuntos de cardinalidade 3 de um conjunto $Q=\left\{t_{1}, \ldots, t_{3 q}\right\}$, onde $s \geq q$, decidir se $C$ contém uma cobertura exata para $Q$, isto é, se existe uma subfamília $C^{\prime} \subseteq C$ tal que cada elemento de $Q$ ocorre em exatamente um elemento de $C^{\prime}$ [GJ79]. Podemos supor que $\bigcup_{i=1}^{s} c_{i}=Q$ para qualquer instância $(C, Q)$ de X3C, pois caso contrário o problema é trivial. É bem conhecido o fato de que X3C é NP-completo [GJ79].

Teorema 3.1.1. A versão de decisão do $\mathrm{PCB}_{2}$ restrito a grafos 2 -conexos é um problema fortemente NP-completo.

Prova: Dada uma instância $(C, Q)$ de X3C, seja $G=(V, E)$ o grafo com conjunto de vértices $V=Q \cup C \cup\left\{p_{1}, p_{2}\right\}$ e conjunto de arestas $E=\left\{p_{1} p_{2}\right\} \cup\left\{p_{i} c_{j}\right.$ : $i=1,2 ; 1 \leq j \leq s\} \cup\left\{c_{j} t_{i}: t_{i} \in c_{j} ; 1 \leq j \leq s\right\} \cup\left\{p_{1} t_{i}: 1 \leq i \leq 3 q\right\}$.

Claramente, $G$ é 2-conexo e pode ser construído em tempo polinomial no tamanho de $(C, Q)$. (Veja um exemplo dessa construção na Figura 3.1, onde $C=$ $\left\{c_{1}, c_{2}, c_{3}, c_{4}\right\}, c_{1}=\left\{t_{1}, t_{2}, t_{4}\right\}, c_{2}=\left\{t_{1}, t_{3}, t_{5}\right\}, c_{3}=\left\{t_{3}, t_{5}, t_{6}\right\}$ e $c_{4}=\left\{t_{2}, t_{4}, t_{6}\right\}$.)

Seja $d:=s-q+1$. Defina uma função peso $w: V \rightarrow \mathbb{Z}_{+}$como segue: $w\left(p_{1}\right)=$ $1+q(3 d+1) ; w\left(p_{2}\right)=d ; w\left(t_{i}\right)=d$ para $i=1, \ldots, 3 q$; e $w\left(c_{j}\right)=1$ para $j=1, \ldots, s$. Notamos que $w(V)=2(3 d q+q+d)$. Provaremos a seguir que $C$ contém uma 
cobertura exata para $Q$ se e somente se $G$ tem uma 2-partição conexa $\left(V_{1}, V_{2}\right)$ tal que $\min \left\{w\left(V_{1}\right), w\left(V_{2}\right)\right\} \geq W / 2$, onde $W:=w(V)$.

Dada uma cobertura exata $C^{\prime}$, construímos uma 2-partição conexa $\left(V_{1}, V_{2}\right)$ de $G$ tomando: $V_{1}=\left\{p_{1}\right\} \cup\left\{c_{j}: c_{j} \notin C^{\prime}\right\}$ e $V_{2}=\left\{p_{2}\right\} \cup\left\{c_{j}, t_{i}: c_{j} \in C^{\prime}\right.$ e $\left.t_{i} \in c_{j}\right\}$. Como $C^{\prime}$ consiste de $q$ subconjuntos, temos que $w\left(V_{1}\right)=w\left(p_{1}\right)+s-q=d+q(3 d+1)=$ $W / 2$.

Reciprocamente, seja $\left(V_{1}, V_{2}\right)$ uma 2-partição conexa de $G$ tal que $\min \left\{w\left(V_{1}\right), w\left(V_{2}\right)\right\} \geq W / 2$. Então $\min \left\{w\left(V_{1}\right), w\left(V_{2}\right)\right\}=W / 2$. Note que, $p_{1}$ and $p_{2}$ não podem pertencer a um mesmo conjunto $V_{i}$, visto que $w\left(p_{1}\right)+w\left(p_{2}\right)=1+d+q(3 d+1)>W / 2$. Suponha, sem perda de generalidade, que $p_{1}$ está em $V_{1}$ e $p_{2}$ está em $V_{2}$. Nenhum vértice de $Q$ está em $V_{1}$, caso contrário $w\left(V_{1}\right) \geq w\left(p_{1}\right)+d=1+q(3 d+1)+d>W / 2$. Então $V_{1}$ contém o vértice $p_{1}$ e alguns vértices de $C$. Como $w\left(V_{1}\right)=W / 2=d+q(3 d+1)$, isto implica que $V_{1}$ contém exatamente $d-1$ vértices de $C$. Como $d-1=s-q$, segue que $V_{2}$ contém precisamente $q$ vértices de $C$. Como estes $q$ vértices são independentes, e os vértices em $Q \cup\left\{p_{2}\right\}$ são igualmente independentes, é fácil verificar que estes $q$ vértices de $C$ pertencentes a $V_{2}$ definem uma cobertura exata para $C$.

Considere agora o problema Partição: dados um conjunto finito $X$ e uma função 'tamanho' $t: X \rightarrow \mathbb{Z}_{+}$, decidir se existe um subconjunto $X^{\prime} \subseteq X$ tal que $\sum_{x \in X^{\prime}} t(x)=\sum_{x \in\left(X \backslash X^{\prime}\right)} t(x)$. Podemos supor, sem perda de generalidade, que $\sum_{x \in X} t(x)$ é par, caso contrário o problema é trivial. Além disto, note que a função $t$ não é limitada polinomialmente (senão, sabemos que o problema poderia ser resolvido em tempo polinomial). Usando este problema - que é sabido ser NP-completo [GJ79] — podemos facilmente demonstrar que a versão de decisão do $\mathrm{PCB}_{2}$ restrito a grafos completos é um problema NP-completo. Note que neste caso, a questão da conexidade pode ser ignorada.

Vamos a seguir investigar a complexidade do $\mathrm{PCB}_{2}$ quando restrito a algumas classes especiais de grafos. Este estudo está relacionado com outros resultados a serem apresentados na Seção 3.3.1.

Definição 3.1.1. Dizemos que um grafo $G$ é um $\Gamma$-grafo se o conjunto de todos os seus pontos de articulação induz um clique.

Nossa motivação para estudar $\Gamma$-grafos advém de algumas topologias de rede 
(WAN, wide area networks) que são casos especiais desta classe de grafos [Tan96].

Definição 3.1.2. Um (w, $\Gamma)$-grafo é um $\Gamma$-grafo $G=(V, E)$ com uma função $w: V \rightarrow \mathbb{Z}_{+}$definida sobre os seus vértices.

Agora considere $(w, \Gamma)$-grafos onde $w(v)=1$ para todo vértice $v$ que não é um ponto de articulação. Provaremos a seguir que o $\mathrm{PCB}_{2}$ restrito a esta classe de grafos é NP-difícil. Lembramos que, de acordo com os resultados mencionados no início da Seção 3.1, se o número de pontos de articulação nos grafos desta classe é $O(\log n)$, onde $n=|V|$, então sabemos que o $\mathrm{PCB}_{2}$ pode ser resolvido em tempo polinomial

Teorema 3.1.2. A versão de decisão do $\mathrm{PCB}_{2}$ restrito à classe dos $(w, \Gamma)$-grafos é um problema NP-completo.

Prova: Dada uma instância $(X, t)$ do problema da Partição, seja $\widehat{X}=\{x \in$ $X \mid t(x)>1\}$ e $G=(V, E)$ o grafo com conjunto de vértices $V=X \cup X^{\prime}$, onde $X^{\prime}=\left\{x^{\prime} \mid x \in \widehat{X}\right\}$, e conjunto de arestas $E=\{x y \mid x, y \in X, x \neq y\} \cup\left\{x x^{\prime} \mid x \in\right.$ $\widehat{X}\}$. Seja $w: V \rightarrow \mathbb{Z}_{+}$a função definida como $w(x)=t(x)-1$ se $x \in \widehat{X}$; e $w(x)=1$, caso contrário. Claramente, $G$ é um $\Gamma$-grafo (com no máximo $|V| / 2$ pontos de articulação).

É fácil verificar que a resposta para uma dada instância $(X, t)$ do problema da Partição é positiva se e somente se $G$ tem uma 2-partição conexa $\left(V_{1}, V_{2}\right)$ tal que $\min \left\{w\left(V_{1}, w\left(V_{2}\right)\right\} \geq w(V) / 2\right.$.

Corolário 3.1.1. A versão de decisão do $\mathrm{PCB}_{2}$ é um problema NP-completo para $(w, \Gamma)$-grafos onde $w(v)=1$ para todo vértice $v$ que não é um ponto de articulação.

Definição 3.1.3. Um $C_{w}$-grafo é um grafo 2-conexo $G=(V, E)$ com uma função $w: V \rightarrow \mathbb{Z}_{+}$tal que o subgrafo induzido pelo conjunto $\{u \in V \mid w(u)>1\}$ é um clique.

Corolário 3.1.2. $O \mathrm{PCB}_{2}$ restrito à classe dos $C_{w}$-grafos é $\mathrm{NP}$-completo.

Prova: Suponha que o $\mathrm{PCB}_{2}$ restrito à classe dos $C_{w}$-grafos possa ser resolvido em tempo polinomial por um algoritmo $\mathcal{A}$. Vamos provar que podemos usar este algoritmo para resolver em tempo polinomial o $\mathrm{PCB}_{2}$ restrito aos $(w, \Gamma)$-grafos 
onde $w(v)=1$ para todo vértice $v$ que não é um ponto de articulação. De fato, dado um tal grafo $(w, \Gamma)$-grafo $G$ basta utilizarmos o algoritmo PartCon descrito a $^{2}$ seguir. Este algoritmo utiliza idéias semelhantes ao algoritmo MaxBal apresentado por Galbiati et. al [GMM97].

$\operatorname{PartCon}_{2}(G, w)$

1. Se $G$ é um grafo 2-conexo devolva a partição encontrada por $\mathcal{A}(G, w)$.

2. Seja $x$ um ponto de articulação de $G$ e sejam $C_{1}, \ldots, C_{h}$ os componentes conexos de $G-x$ obtidos ao remover $x$ de $G$. Seja $w\left(C_{i}\right)$ a soma dos pesos dos vértices de $C_{i}, i=1, \ldots, h$. Sem perda de generalidade, seja $C_{1}$ um componente que maximiza $w\left(C_{i}\right)$.

3. Se $w\left(C_{1}\right) \leq\left\lfloor\frac{W}{2}\right\rfloor$ então devolva a 2-partição $\left(V_{1}, V_{2}\right)$, onde $V_{1}=V\left(C_{1}\right)$ e $V_{2}=V \backslash V_{1}$.

4. Senão

- Seja $G^{\prime}$ o subgrafo de $G$ induzido por $x$ e pelos vértices de $C_{1}$; e seja $w^{\prime}$ a nova função peso assim definida: $w^{\prime}(x)=w(x)+\sum_{i=2}^{h} w\left(C_{i}\right)$ e $w^{\prime}(v)=w(v)$ para $v \in V\left(C_{1}\right) . / * G^{\prime}$ tem menos pontos de articulação do que $G * 1$

- $\left(V_{1}^{\prime}, V_{2}^{\prime}\right)=\operatorname{PartCon}_{2}\left(G^{\prime}, w^{\prime}\right)$.

- Transforme $\left(V_{1}^{\prime}, V_{2}^{\prime}\right)$ em uma 2-partição conexa $\left(V_{1}, V_{2}\right)$ para $G$ substituindo cada vertice $x^{\prime}$ pelos vértices que definem $w^{\prime}\left(x^{\prime}\right)$.

- Devolva $\left(V_{1}, V_{2}\right)$.

Note que, no passo 1 , se o grafo é 2 -conexo então este é um $C_{w}$-grafo.

Como o algoritmo acima resolve em tempo polinomial o $\mathrm{PCB}_{2}$ restrito a $(w, \Gamma)$ grafos onde $w(v)=1$ para cada vértice $v$ que não é um ponto de articulação, então temos uma contradição ao Corolário 3.1.1.

Os resultados acima mencionados dizem respeito à dificuldade de se resolver o $\mathrm{PCB}_{2}$ (exatamente); um outro aspecto interessante é a dificuldade de se obter soluções aproximadas para este problema. Sob esta perspectiva, estamos interessados em determinar limites de aproximabilidade para este problema. Os resultados nessa linha serão apresentados na próxima seção. 


\subsection{Dificuldade de Aproximação}

Investigações a respeito de limites de aproximabilidade para o $\mathrm{PCB}_{2}$ foram feitas inicialmente em 1996 por Chlebíková [Chl96], que provou o seguinte resultado.

Teorema 3.2.1 (Chlebíková, 1996). Para qualquer racional $\delta>0$, é NP-difícil achar em tempo polinomial uma solução para o $\mathrm{PCB}_{2}$ com uma garantia de erro absoluto de $n^{1-\delta}$, onde $n$ é o número de vértices do grafo $G$ de entrada. Esta afirmação vale também para grafos bipartidos e sem pesos.

Observe que a medida de aproximação mencionada no resultado acima é o erro absoluto (veja Definição 2.4.1). Uma questão interessante é saber o que ocorre se considerarmos como medida a razão de aproximação (veja Definição 2.3.2). Pelo que foi discutido na Seção 2.4, sabemos que a qualidade da aproximação depende da medida de aproximação utilizada. Trabalhamos nesta direção e obtivemos o resultado apresentado a seguir.

$\mathrm{Na}$ prova abaixo utilizamos uma versão restrita de X3C, chamado problema da Cobertura Exata (CE), que é sabidamente NP-completo [PY82]: dada uma família $C=\left\{C_{1}, C_{2}, \ldots, C_{3 q}\right\}$ de subconjuntos de cardinalidade 3 de um conjunto $X=\left\{x_{1}, x_{2}, \ldots, x_{3 q}\right\}$, tal que cada elemento em $X$ ocorre exatamente 3 vezes nos conjuntos $C_{1}, C_{2}, \ldots, C_{3 q}$, decidir se existe uma subfamília $C^{\prime}=\left\{C_{j_{1}}, C_{j_{2}}, \ldots, C_{j_{q}}\right\}$ de $C$ que cobre $X$ exatamente.

Teorema 3.2.2. O problema Max 2-Partição Conexa Balanceada sobre grafos com $n$ vértices não admite um algoritmo $(1+\epsilon)$-aproximado, onde $\epsilon \leq \frac{1}{n^{2}}$, a menos que $\mathrm{P}=\mathrm{NP}$ (mesmo para grafos bipartidos).

Prova: Suponha que exista um algoritmo $\mathcal{A}$ para o $\mathrm{PCB}_{2}$ sobre grafos com $n$ vértices que é uma $(1+\epsilon)$-aproximação, onde $\epsilon \leq \frac{1}{n^{2}}$. Mostraremos que este algoritmo pode ser usado para resolver o problema da Cobertura Exata, obtendo assim uma contradição, a menos que $\mathrm{P}=\mathrm{NP}$.

Seja $(C, X)$ uma instância do problema da Cobertura Exata onde $\mathcal{C}=$ $\left\{C_{1}, C_{2}, \ldots, C_{3 q}\right\}$ é uma família de subconjuntos de $X=\left\{x_{1}, x_{2}, \ldots, x_{3 q}\right\}$. Construa a partir de $(C, X)$ um grafo $G=(V, E)$ da seguinte maneira.

Tome $V=C \cup X \cup\{a, b\}$ e $E=\bigcup_{j=1}^{3 q}\left[\left\{C_{j} x_{i} \mid x_{i} \in C_{j}\right\} \cup\left\{C_{j} a\right\} \cup\left\{C_{j} b\right\}\right]$. Atribua aos vértices $v$ de $G$ pesos $w(v)$ da seguinte maneira: $w(a)=6 q^{3}+q^{2} ; w(b)=2 q^{2}$; 
$w\left(C_{j}\right)=q$ para $j=1, \ldots, 3 q$; e $w\left(x_{i}\right)=2 q^{2}$ para $i=1, \ldots, 3 q$. Observe que $w(V)=2\left(6 q^{3}+3 q^{2}\right)$ e $|V|=6 q+2$.

Lembramos que a medida de uma 2-partição $\left(V_{1}, V_{2}\right)$ de $G$ é o valor $\min \left\{w\left(V_{1}\right), w\left(V_{2}\right)\right\}$.

Afirmação 1. Se existe uma subfamília $C^{\prime}$ de $C$ que cobre $X$ exatamente, então $G$ tem uma 2-partição conexa com medida $w(V) / 2$.

Prova: Dada uma cobertura exata $C^{\prime}$ de $X$, podemos construir a seguinte 2partição conexa $\left(V_{1}, V_{2}\right)$ de $G: V_{1}=\{a\} \cup\left(C \backslash C^{\prime}\right)$ e $V_{2}=\{b\} \cup X \cup C^{\prime}$.

Claramente, temos que $w\left(V_{1}\right)=6 q^{3}+3 q^{2}=w(V) / 2=w\left(V_{2}\right)$.

Afirmação 2. Se $G$ tem uma 2-partição conexa $\left(V_{1}, V_{2}\right)$ cuja medida é pelo menos $w(V) / 2-q+1$, então existe uma subfamília de $C$ que cobre $X$ exatamente.

Prova: Seja $\left(V_{1}, V_{2}\right)$ uma 2-partição conexa de $G$ com medida $m$, tal que $m \geq$ $w(V) / 2-q+1$.

Observe que $a$ e $b$ não podem pertencer a um mesmo conjunto $V_{i}(i=1,2)$. Caso contrário, $a, b$ e $C_{j}$ para algum $j \in\{1,2, \ldots, 3 q\}$ pertenceriam a um mesmo conjunto, e assim, o peso deste conjunto seria pelo menos $6 q^{3}+3 q^{2}+q=w(V) / 2+q$. Mas, então $m \leq w(V) / 2-q$, uma contradição.

Logo, suponha que $a \in V_{1}$ e $b \in V_{2}$. Usando um argumento de contagem similar podemos provar que $X \subseteq V_{2}$. Assim, pela conexidade de subgrafo induzido por $V_{2}$, temos que $\left|C \cap V_{2}\right| \geq q$. Mas $\left|C \cap V_{2}\right|>q$ implica que $\left|C \cap V_{1}\right| \leq 2 q-1$. E neste caso, $w\left(V_{1}\right) \leq 6 q^{3}+q^{2}+(2 q-1) q=6 q^{3}+3 q^{2}-q=w(V) / 2-q$, e temos novamente uma contradição. Então $\left|C \cap V_{2}\right|=q$, e portanto $C \cap V_{2}$ cobre $X$ exatamente.

Vamos agora mostrar que, usando o algoritmo $\mathcal{A}$ (mencionado no início da prova), podemos resolver o problema CE. Seja $\mathcal{A}_{C E}$ o seguinte algoritmo para CE. Dada uma instância $(C, X)$, construa o grafo $G$, como descrito anteriormente. Agora, aplique o algoritmo $\mathcal{A} \mathrm{em} G$. Se este algoritmo encontrar uma 2-partição conexa com medida pelo menos $w(V) / 2-q+1$, então devolva a mensagem " $C$ tem uma cobertura exata." Se a 2-partição encontrada tiver medida menor que $w(V) / 2-q+1$, então devolva a mensagem "C não tem uma cobertura exata."

Vamos mostrar que $\mathcal{A}_{C E}$ de fato resolve o CE. Suponha que $C$ tenha uma cobertura exata. Então, pela Afirmação 1, $G$ tem uma 2-partição conexa com 
medida $w(V) / 2$. Como o algoritmo $\mathcal{A}$ é uma $(1+\epsilon)$-aproximação, onde $\epsilon \leq \frac{1}{n^{2}} \mathrm{e}$ $n=|V|$, então $\mathcal{A}$ devolve uma solução com medida $m \geq\left(n^{2} /\left(n^{2}+1\right)\right)$ opt. Como opt $=w(V) / 2$, segue que

$$
m \geq\left(\frac{n^{2}}{n^{2}+1}\right) \frac{w(V)}{2}=\left(1-\frac{1}{n^{2}+1}\right) \frac{w(V)}{2}=\frac{w(V)}{2}-\frac{w(V)}{2\left(n^{2}+1\right)}
$$

Como $n=6 q+2$ e $w(V)=2\left(6 q^{3}+3 q^{2}\right)$, temos que $\frac{w(V)}{2\left(n^{2}+1\right)}<q$, donde segue que $m \geq w(V) / 2-q+1$.

Suponha agora que $C$ não tenha uma cobertura exata. Então pela Afirmação 2 o grafo $G$ só tem soluções com medida menor do que $w(V) / 2-q+1$. Neste caso, obviamente o algoritmo $\mathcal{A}$ devolve uma solução com medida menor do que $w(V) / 2-q+1$. Com isso, fica provado que o algoritmo $\mathcal{A}_{C E}$ resolve o problema $\mathrm{CE}$.

Como a construção de $G$ pode ser feita em tempo polinomial no tamanho de $(C, X)$, e o algoritmo $\mathcal{A}$ é polinomial no tamanho de $G$, segue que $\mathcal{A}_{C E}$ é polinomial no tamanho de $(C, X)$. Isso completa a prova do teorema.

Corolário 3.2.1. O problema Max 2-Partição Conexa Balanceada não possui FPTAS (mesmo para grafos bipartidos), a menos que $\mathrm{P}=\mathrm{NP}$.

Prova: Suponha que exista um FPTAS para o $\mathrm{PCB}_{2}$. Por definição, sabemos que para qualquer $r>1$ existe um algoritmo $\mathcal{A}_{r}$ que é uma $r$-aproximação para o $\mathrm{PCB}_{2}$ e é polinomial no tamanho da instância e em $1 /(r-1)$.

Assim, dada uma instância do $\mathrm{PCB}_{2}$, considere $r=\left(n^{2}+1\right) / n^{2}$, onde $n$ é o número de vértices do grafo. Neste caso, $\mathcal{A}_{r}$ é um algoritmo $\left(1+\frac{1}{n^{2}}\right)$-aproximado para o $\mathrm{PCB}_{2}$ (note que ele é polinomial no tamanho da instância), o que contradiz o Teorema 3.2.2.

É importante destacar que o resultado mencionado no Corolário 3.2.1 já era conhecido [CGM83, DF85]. Tal resultado segue do fato de que a versão de decisão do $\mathrm{BCP}_{2}$ (para grafos conexos) é um problema fortemente NP-completo. Observamos que o resultado exibido no Teorema 3.2 .2 mostra a dificuldade de se ter 
uma aproximação de certa forma 'menos exigente' (mesmo para grafos com pesos polinomialmente limitados).

Vimos no Teorema 3.1.1 que a versão de decisão do $\mathrm{PCB}_{2}$ restrito a grafos 2-conexos é um problema fortemente NP-completo. Assim, o correspondente resultado de inaproximabilidade - para grafos 2-conexos (não necessariamente bipartidos) - que segue como corolário imediato deste fato é o seguinte.

Corolário 3.2.2. Para grafos 2-conexos, Max 2-Partição Conexa Balanceada não admite um FPTAS, a menos que $\mathrm{P}=\mathrm{NP}$.

Prova: Suponha que o $\mathrm{PCB}_{2}$ restrito a grafos 2-conexos admita um FPTAS digamos $\mathcal{A}$. Seja $x$ uma instância de $\mathrm{PCB}_{2}$, a qual consiste de um grafo 2-conexo $G=(V, E)$ e uma função $w: V \rightarrow \mathbb{Z}_{+}$. Então, $\operatorname{maxint}(x) \leq w(V)=W$.

Dado $\mathcal{A}$, podemos exibir um algoritmo pseudo-polinomial $\mathcal{A}^{\prime}$ que resolve qualquer instância $x$ de $\mathrm{PCB}_{2}$. Este algoritmo é simplesmente definido como: $\mathcal{A}^{\prime}(x)=$ $\mathcal{A}(x, r)$, onde $r=1+\frac{1}{W}$ é a aproximação exigida.

Seja $m$ a medida da solução devolvida pelo algoritmo $\mathcal{A}^{\prime}$. Se opt $(x)$ é a medida de uma solução ótima para $x$, então temos que

$$
\operatorname{opt}(x) \leq\left(1+\frac{1}{W}\right) m . \text { Assim, opt }(x)-m \leq \frac{m}{W}<1,
$$

onde a última inequação ocorre porque $m \leq \operatorname{opt}(x) \leq W / 2$. Visto que opt $(x)$ e $m$ são inteiros, temos que opt $(x)=m$. Quanto ao tempo de execução, lembramos que $\mathcal{A}$ tem complexidade de tempo limitado por $q(1 /(r-1),|x|)$, para algum polinômio $q$. Portanto, $\mathcal{A}^{\prime}$ tem complexidade de tempo limitado por $q(W,|x|)$, isto é, polinomial em ambos $|x|$ e maxint $(x)$.

Por outro lado, sabemos que se $\mathrm{P} \neq \mathrm{NP}$ então nenhum problema fortemente NP-difícil pode ter um algoritmo pseudo-polynomial [GJ76]. Assim, usando este resultado e o Teorema 3.1.1 obtemos uma contradição quanto à existência do algoritmo pseudo-polinomial que exibimos anteriormente.

Tendo apresentado resultados a respeito da complexidade computacional e limites de aproximabilidade do $\mathrm{PCB}_{2}$, exibimos a seguir resultados positivos quanto às propriedades de aproximação desse problema. 


\subsection{Aproximabilidade}

O problema Max 2-Partição Conexa Balanceada restrito a grafos completos é equivalente a uma variante do Max Mochila denominada Max Soma de Subconjuntos, onde os lucros e os pesos são iguais. Neste caso, o limite utilizado é a metade da soma dos pesos de todos os vértices. Como Max Mochila tem um FPTAS [IK75, Law79], segue que o $\mathrm{PCB}_{2}$ tem um FPTAS [IK75, Law79] para esta classe de grafos.

Para grafos conexos (e 2-conexos), o melhor resultado conhecido para o $\mathrm{PCB}_{2}$ é um algoritmo de aproximação obtido por Chlebíková [Chl96] que resolve o problema dentro de uma razão de $\frac{4}{3}$. Nesta tese, apresentamos uma releitura deste algoritmo e exibimos uma análise parametrizada que nos permite descrever classes de grafos para as quais o algoritmo devolve uma solução que se aproxima assintoticamente do ótimo.

Primeiramente exibiremos um algoritmo para o $\mathrm{PCB}_{2}$ restrito a grafos 2conexos. Como veremos, este algoritmo será usado como rotina no algoritmo para grafos conexos.

Antes de descrevermos os algoritmos, vejamos uma definição e um lema auxiliar.

Definição 3.3.1. Seja $G$ um grafo 2-conexo e $\left(V_{1}, V_{2}\right)$ uma 2-partição conexa de $G$. Dizemos que um vértice $u$ de $V_{2}$ é admissível (para $\left.V_{1}\right)$ se $\left(V_{1} \cup\{u\}, V_{2} \backslash\{u\}\right)$ também é uma 2-partição conexa de $G$.

Lema 3.3.1. Seja $G=(V, E)$ um grafo 2-conexo e $\left(V_{1}, V_{2}\right)$ uma 2-partição conexa de $G$ tal que $\left|V_{2}\right| \geq 2$. Então existem pelo menos dois vértices distintos $v^{\prime}$ e $v^{\prime \prime}$ em $V_{2}$ que são admissíveis (para $V_{1}$ ).

Prova: Seja $G_{2}$ o subgrafo de $G$ induzido por $V_{2}$. Se $G_{2}$ é 2-conexo o resultado segue trivialmente. Pelo fato de $G$ ser 2-conexo.

Suponha então que $G_{2}$ não seja 2-conexo e considere $H$ o grafo de blocos de $G_{2}$. Seja $A$ o conjunto dos pontos de articulação de $G_{2}$ e $B$ o conjunto dos seus blocos. Como definido na Seção 2.1, o grafo $H$ é formado pelas arestas $a b$ onde $a \in A, b \in B$ e $a$ é um ponto de articulação de $b$. Claramente, $H$ é uma árvore. Chame de blocos terminais os blocos correspondentes às folhas de $H$. Como $H$ é uma árvore não-trivial, sabemos que existem pelo menos dois deles. 
Claramente, em $G$ os vértices que não são pontos de articulação e que pertencem a blocos distintos não são adjacentes.

Seja $b$ um bloco terminal e $a$ o ponto de articulação de $V_{2}$ tal que $a b$ é uma aresta de $H$. Se em $G$ os vértices distintos de $a$, pertencentes ao bloco $b$, não fossem adjacentes a vértices de $V_{1}$, então $a$ seria um ponto de articulação em $G$. Uma contradição, pois $G$ é 2-conexo.

Logo, os blocos terminais são adjacentes a $V_{1}$ : existe em cada bloco terminal pelo menos um vértice adjacente a $V_{1}$, que não é um ponto de articulação de $G_{2}$. Como temos pelo menos dois blocos terminais em $H$, isto conclui nossa demonstração, pois temos dois vértices (pertencentes a blocos terminais distintos) que são admissíveis (para $V_{1}$ ).

A seguir, descrevemos o algoritmo BalBicon 2 para o $\mathrm{PCB}_{2}$ restrito a grafos 2-conexos. Dado um tal grafo $G=(V, E)$ e $w: V \rightarrow \mathbb{Z}_{+}$, este algoritmo primeiramente determina um vértice, digamos $v_{1}$, de peso máximo. Se $w\left(v_{1}\right)>(1 / 2) w(V)$, o algoritmo devolve a partição $\left(V_{1}, V_{2}\right)$, onde $V_{1}=\left\{v_{1}\right\}$ e $V_{2}=V \backslash V_{1}$. Caso contrário, inicia com esta partição conexa, e para atualizá-la procura em $V_{2}$ um vértice admissível com o menor peso possível. O algoritmo pára quando a adição de qualquer novo vértice a $V_{1}$ não melhora a medida da solução.

Algoritmo $\mathrm{BalBicon}_{2}(G, w)$

Entrada: um grafo 2-conexo $G=(V, E)$ e uma função peso $w: V \rightarrow \mathbb{Z}_{+}$.

Saída: uma 2-partição conexa $\left(V_{1}, V_{2}\right)$ de $G$.

1. Seja $v_{1}=\arg \max \{w(v) \mid v \in V\}$.

2. Sejam $V_{1}=\left\{v_{1}\right\}, V_{2}=V \backslash V_{1}$ e $\beta=w(V) / 2$.

3. Se $w\left(v_{1}\right) \geq \beta$ então devolva $\left(V_{1}, V_{2}\right)$ e pare.

4. Enquanto $w\left(V_{1}\right)<\beta$ faça

4.1 Escolha $u \in V_{2}$ tal que $u$ é admissível e de peso mínimo.

4.2 Se $w(u) \geq 2\left(\beta-w\left(V_{1}\right)\right)$ então /* CASO A: $w\left(V_{1}\right) \geq \beta-\frac{1}{2} w(u)$. $/$ pare.

4.3 Senão /* CASo B */

$V_{1}=V_{1} \cup\{u\}$ e $V_{2}=V_{2} \backslash\{u\}$.

$/^{*}$ Aqui temos $w\left(V_{1}\right)<\beta+\frac{1}{2} w(u) . * /$ 
5. Devolva $\left(V_{1}, V_{2}\right)$.

\section{fim_do_Algoritmo}

Antes de analisarmos a razão de aproximação do algoritmo BalBicon ${ }_{2}$, enunciamos a seguinte observação cuja prova é imediata.

Lema 3.3.2. Seja $V=\left\{v_{1}, v_{2}, \ldots, v_{n}\right\}$ e $w: V \rightarrow \mathbb{Z}_{+}$. Se $w\left(v_{1}\right) \geq w\left(v_{2}\right) \geq \ldots \geq$ $w\left(v_{n}\right)$ e $t=w(V) / w\left(v_{k}\right)$, onde $1 \leq k \leq n$, então $t \geq k$.

Teorema 3.3.1. Seja I uma instância do $\mathrm{PCB}_{2}$ que consiste de um grafo 2-conexo $G=(V, E)$ e uma função $w: V \rightarrow \mathbb{Z}_{+}$. Seja $V=\left\{v_{1}, v_{2}, \ldots, v_{n}\right\}, n \geq 3$, $w\left(v_{1}\right) \geq w\left(v_{2}\right) \geq \ldots \geq w\left(v_{n}\right)$ e $t:=w(V) / w\left(v_{3}\right)$. Então o algoritmo BalBicon 2 , aplicado à instância $I$, devolve em tempo polinomial uma 2-partição conexa de $G$ com medida $m$, tal que

(1) Se $w\left(v_{1}\right) \geq \frac{1}{2} w(V)$ então $m=\operatorname{opt}(I)$.

(2) Se $w\left(v_{1}\right) \leq \frac{1}{2} w(V)$ então $m \geq \frac{1}{2}\left[w(V)-w\left(v_{3}\right)\right] \geq \frac{t-1}{2 t} w(V)$.

(3) $\frac{\operatorname{opt}(I)}{m} \leq \frac{2 t-4}{t-1}$, se $3 \leq t \leq 4 ; \quad$ e $\frac{\operatorname{opt}(I)}{m} \leq \frac{t}{t-1}$, se $t \geq 4$.

Prova: É imediato que o algoritmo BalBicon $_{2}$ devolve em tempo polinomial uma partição conexa de $G$. A afirmação (1) também é óbvia.

Vamos provar a afirmação (2). Suponhamos que $w\left(v_{1}\right)<\frac{1}{2} w(V)$. Observe que qualquer vértice $u$ escolhido no passo 3.3 satisfaz $w(u) \leq w\left(v_{3}\right)$, visto que $v_{1} \notin V_{2}$ e $V_{2}$ tem pelo menos dois vértices admissíveis (veja Lema 3.3.1).

Temos duas situações para analisar, correspondentes ao último caso (CAso A ou CAso B) que ocorreu antes da devolução da solução $\left(V_{1}, V_{2}\right)$. Seja $\beta=\frac{1}{2} w(V)$, como definido no algoritmo.

CAso A: Neste caso, $w(u) \geq 2\left(\beta-w\left(V_{1}\right)\right)$, e portanto $w\left(V_{1}\right) \geq \beta-\frac{1}{2} w(u)$. Logo, $m \geq \beta-\frac{1}{2} w(u) \geq \beta-\frac{1}{2} w\left(v_{3}\right)$, visto que $w(u) \leq w\left(v_{3}\right)$. Como $w\left(v_{3}\right)=\frac{w(V)}{t}$, então

$$
m \geq \beta-\frac{1}{2 t} w(V)=\frac{1}{2} w(V)-\frac{1}{2 t} w(V)=\left(\frac{1}{2}-\frac{1}{2 t}\right) w(V)=\left(\frac{t-1}{2 t}\right) w(V) .
$$


CAso B: Se este foi o último caso ocorrido, então $\beta \leq w\left(V_{1}\right)<\beta+\frac{1}{2} w(u)$. Logo,

$$
m=w\left(V_{2}\right)=w(V)-w\left(V_{1}\right)>w(V)-\beta-\frac{1}{2} w(u)=\frac{1}{2} w(V)-\frac{1}{2} w(u) .
$$

Como $w(u) \leq w\left(v_{3}\right)$, e $w\left(v_{3}\right)=\frac{w(V)}{t}$, temos que

$$
m>\frac{1}{2} w(V)-\frac{1}{2} w\left(v_{3}\right)=\frac{1}{2} w(V)-\frac{1}{2 t} w(V)=\left(\frac{t-1}{2 t}\right) w(V) .
$$

Se $w\left(v_{1}\right)=\frac{1}{2} w(V)$, a afirmação (2) é claramente verdadeira. Com isso, a prova da afirmação (2) está completa.

Vamos agora provar a afirmação (3). É imediato que opt $(I) \leq \frac{1}{2} w(V)$. Contudo, podemos melhorar este limite para opt $(I) \leq\left(1-\frac{2}{t}\right) w(V)$, se $3 \leq t \leq 4$. De fato, para qualquer partição $(X, Y)$ de $V$, um entre $X$ e $Y$ contém pelo menos dois elementos de $\left\{v_{1}, v_{2}, v_{3}\right\}$. Logo, o peso de tal conjunto é pelo menos $\frac{2}{t} w(V) \geq$ $\frac{1}{2} w(V)$. Assim, opt $(I) \leq w(V)-\frac{2}{t} w(V)=\left(1-\frac{2}{t}\right) w(V)$, como afirmamos.

Separando em dois casos, temos:

(a) $3 \leq t \leq 4$. Neste caso, usando o limite melhorado para o valor ótimo, temos que $\operatorname{opt}(I) \leq\left(1-\frac{2}{t}\right) w(V)$. Assim, pelo que foi demonstrado no item 2, concluímos que

$$
\operatorname{opt}(I) \leq\left(1-\frac{2}{t}\right)\left(\frac{2 t}{t-1}\right) m=\left(\frac{t-2}{t}\right)\left(\frac{2 t}{t-1}\right) m=\frac{2 t-4}{t-1} m
$$

(b) $t \geq 4$. Neste caso, usando o limite trivial para o valor ótimo juntamente com o resultado mostrado no item 2 , deduzimos que

$$
\operatorname{opt}(I) \leq \frac{1}{2}\left(\frac{2 t}{t-1}\right) m=\left(\frac{t}{t-1}\right) m
$$

Com isso, está completa a prova do teorema.

No que segue, exibimos uma instância $I$ do $\mathrm{PCB}_{2}$ para mostrar que a razão

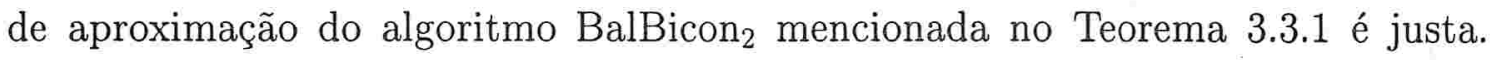
Considere o grafo 2-conexo $G=(V, E)$ da Figura 3.2 com a seguinte função peso 


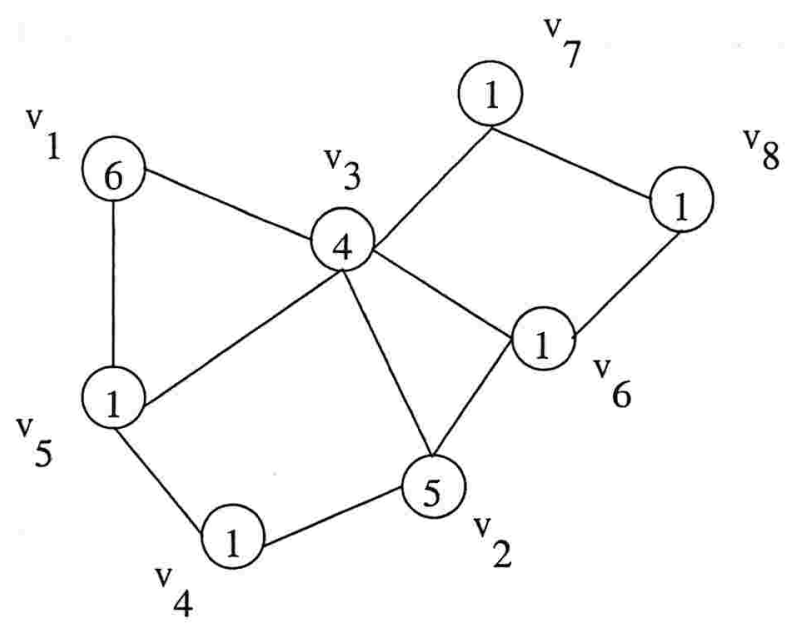

Figura 3.2: Grafo 2-conexo com pesos nos vértices.

definida sobre seus vértices: $w\left(v_{1}\right)=6, w\left(v_{2}\right)=5, w\left(v_{3}\right)=4, w\left(v_{4}\right)=w\left(v_{5}\right)=$ $w\left(v_{6}\right)=w\left(v_{7}\right)=w\left(v_{8}\right)=1$. Então, $w(V)=20, \beta=10$ e $t=5$.

$\mathrm{O}$ algoritmo BalBicon $_{2}$ aplicado à instância $I$ exibida na Figura 3.2 executa 3 iterações e obtém os valores exibidos na Tabela 3.2. Portanto, devolve a 2-partição conexa $\left(V_{1}, V_{2}\right)$, onde $V_{1}=\left\{v_{1}, v_{5}, v_{4}\right\}$ e $V_{2}=V \backslash V_{1}$, com medida $m=8$. É fácil ver que $\operatorname{opt}(I)=10$. Neste caso, $\frac{\text { opt }(I)}{m}=\frac{10}{8}=\frac{t}{t-1}$.

\begin{tabular}{|c|c|c|}
\hline \hline Iteração & $V_{1}$ & $u$ \\
\hline \hline 1 & $v_{1}$ & $v_{5}$ \\
\hline 2 & $v_{1}, v_{5}$ & $v_{4}$ \\
\hline 3 & $v_{1}, v_{5}, v_{4}$ & $v_{3}$ \\
\hline
\end{tabular}

Tabela 3.2: Iterações executadas pelo algoritmo BalBicon $_{2}$.

Corolário 3.3.1. O algoritmo BalBicon $_{2}$ é uma $4 / 3$-aproximação para o $\mathrm{PCB}_{2}$ restrito a grafos 2-conexos. Ademais, essa razão 4/3 é justa.

Prova: O resultado segue aplicando-se o Teorema 3.3.1 juntamente com o fato de que para qualquer instância temos que $t \geq 3$ (veja o Lema 3.3.2).

Para verificar que a razão é justa, considere o grafo 2-conexo $G=(V, E)$ indicado na Figura 3.3. Note que $w(V)=24, \beta=12$ e $t=4$. O algoritmo BalBicon 2 
executa as 3 iterações descritas na Tabela 3.3 e obtém uma 2-partição conexa com medida $m=9$. Como opt $(I)=12$, temos que $\frac{o p t(I)}{m}=\frac{12}{9}=\frac{4}{3}$.

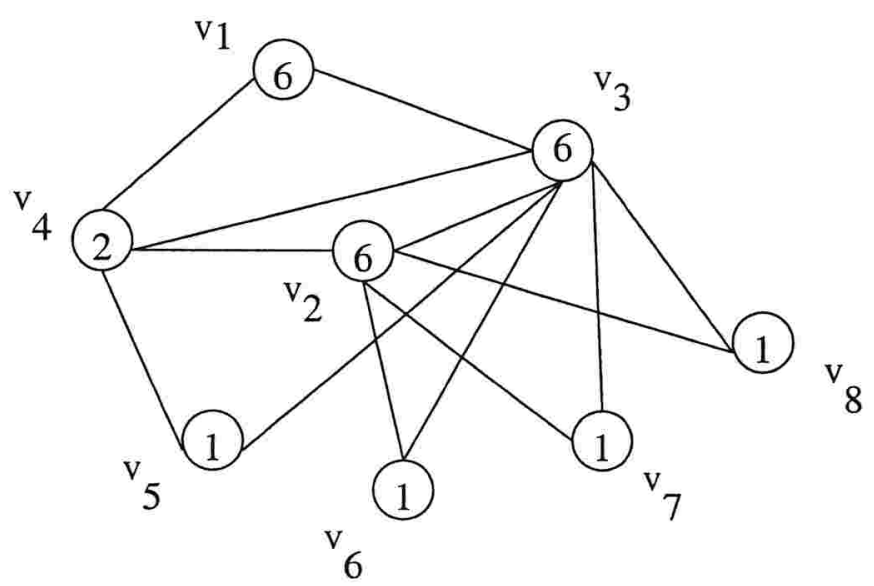

Figura 3.3: Grafo 2-conexo com pesos nos vértices.

\begin{tabular}{|c|c|c|}
\hline \hline Iteração & $V_{1}$ & $u$ \\
\hline \hline 1 & $v_{1}$ & $v_{4}$ \\
\hline 2 & $v_{1}, v_{4}$ & $v_{5}$ \\
\hline 3 & $v_{1}, v_{4}, v_{5}$ & $v_{3}$ \\
\hline
\end{tabular}

Tabela 3.3: Iterações executadas pelo algoritmo BalBicon $_{2}$.

O Teorema 3.3.1 nos permite concluir que para instâncias do $\mathrm{PCB}_{2}$ nas quais, excetuando o primeiro e o segundo vértices de maior peso, os demais vértices têm peso bem pequeno relativamente ao peso total $(w(V))$, o algoritmo BalBicon 2 devolve soluções bem próximas do ótimo. Note que, quanto maior o valor de $t$, definido neste teorema, melhor é a razão de aproximação.

Com relação ao BalBicon $_{2}$ podemos também concluir o seguinte resultado.

Teorema 3.3.2. Seja I uma instância do $\mathrm{PCB}_{2}$ que consiste de um grafo 2-conexo $G=(V, E)$ e uma função $w: V \rightarrow \mathbb{Z}_{+}$. Seja $v_{1}$ um vértice de $V$ tal que $w\left(v_{1}\right)=$ $\max \{w(v) \mid v \in V\}$. Se $w\left(v_{1}\right) \leq \frac{1}{2} w(V)$, então o algoritmo BalBicon ${ }_{2}$, aplicado à instância I, devolve em tempo polinomial uma 2-partição conexa cuja medida é pelo menos $(1 / 3) w(V)$. 
Prova: Seja $V=\left\{v_{1}, v_{2}, \ldots, v_{n}\right\}$ e suponha que $w\left(v_{1}\right) \geq w\left(v_{2}\right) \geq \ldots \geq w\left(v_{n}\right)$. Seja $t:=w(V) / w\left(v_{3}\right)$. Então pelo Lema 3.3.2, temos que $t \geq 3$. Assim, se $m$ é a medida da solução devolvida pelo algoritmo, pelo Teorema 3.3.1 (item (2)) temos que

$$
m \geq \frac{t-1}{2 t} w(V) \geq \frac{1}{3} w(V) .
$$

Até o momento focamos no problema Max 2-Partição Conexa Balanceada restrito a grafos 2-conexos. Veremos a seguir que também é possível resolver o $\mathrm{PCB}_{2}$ para qualquer grafo conexo dentro da mesma razão 4/3 [Chl96]. Para isto necessitamos dos resultados apresentados a seguir.

Definição 3.3.2. Seja $G=(V, E)$ um grafo conexo e $w: V \rightarrow \mathbb{Z}_{+}$. Seja $A_{G}$ o conjunto dos pontos de articulação de $G$ e $H_{G}$ o conjunto dos blocos de $G$. Denotamos por $T_{G}=\left(V\left(T_{G}\right), E\left(T_{G}\right)\right)$ a árvore de blocos de $G$ (veja Seção 2.1), onde $V\left(T_{G}\right)=A_{G} \cup H_{G}$ e $E\left(T_{G}\right)=\left\{\{a, H\} \mid H \in H_{G}\right.$ e $\left.a \in V(H) \cap A_{G}\right\}$.

Para $H \in H_{G}$, e $v \in V(H) \cap A_{G}$, seja $B(H, v)=\left\{\cup V(\tilde{H}) \mid \tilde{H} \in H_{G}\right.$ e $\tilde{H}$ pertence à subárvore de $T_{G}-\{v, H\}$ que contém $\left.v\right\}$.

Para cada bloco $H$, definimos uma função peso $w_{H}$ nos vértices de $H$ como segue:

$$
w_{H}(v)= \begin{cases}w(v) & \text { se } v \in V(H) \backslash A_{G} \\ \left\{\sum_{u} w(u) \mid u \in B(H, v)\right\} & \text { se } v \in V(H) \cap A_{G}\end{cases}
$$

Mencionamos a seguir dois lemas cujas provas são bem simples e serão deixadas a cargo do leitor.

Lema 3.3.3. Seja $G=(V, E)$ um grafo conexo com $|V| \geq 2$ e $\left(V_{1}, V_{2}\right)$ uma 2partição conexa de $G$. Então existe um único bloco $H=(V(H), E(H))$ de $G$ tal que ambos $V_{1} \cap V(H)$ e $V_{2} \cap V(H)$ não são vazios. Mais ainda, $\left(V_{1} \cap V(H), V_{2} \cap V(H)\right)$ é uma 2-partição conexa de $H$.

Lema 3.3.4. Seja $G=(V, E)$ um grafo conexo com $|V| \geq 2, H$ um bloco de $G$ e $\left(\tilde{V}_{1}, \tilde{V}_{2}\right)$ uma 2-partição conexa de $H$. Então existe uma única 2-partição conexa $\left(V_{1}, V_{2}\right)$ de $G$ tal que $\tilde{V}_{1}=V_{1} \cap V(H)$ e $\tilde{V}_{2}=V_{2} \cap V(H)$. 
Estes lemas indicam que podemos resolver o problema $\mathrm{PCB}_{2}$ sobre qualquer grafo conexo reduzindo-o a problemas do mesmo tipo em seus blocos (estes com pesos ajustados). Esta redução (ajustamento dos pesos nos blocos) e o algoritmo BalBicon $_{2}$ é a base do algoritmo BalCon 2 , que preserva a aproximação garantida pelo algoritmo BalBicon $_{2}$ que vimos para grafos 2-conexos.

Dado um grafo conexo $G$, o algoritmo $\mathrm{BalCon}_{2}$ primeiramente encontra os conjuntos $A_{G}$ e $H_{G}$ dos pontos de articulação e blocos de $G$, respectivamente, e constrói o grafo de blocos de $G$. Depois, determina para cada bloco $H \in H_{G}$ a correspondente função peso $w_{H}$, e aplica o algoritmo $\mathrm{BalBicon}_{2}$ ao par $\left(H, w_{H}\right)$ devolvendo $\left(V_{1}^{H}, V_{2}^{H}\right)$, uma aproximação da máxima partição conexa balanceada (com relação a $w_{H}$ ) para $H$. Finalmente, entre os blocos $H \in H_{G}$ escolhe $H_{0} \in H_{G}$ cuja partição conexa $\left(V_{1}^{H_{0}}, V_{2}^{H_{0}}\right)$, devolvida pelo algoritmo $\mathrm{BalBicon}_{2}$, tem medida máxima. Tal partição se estende de maneira única a uma partição conexa $\left(V_{1}, V_{2}\right)$ de $G$ (veja Lema 3.3.4), que é então devolvida pelo algoritmo.

Algoritmo $\mathrm{BalCon}_{2}(G, w)$

Entrada: um grafo conexo $G=(V, E)$ e $w: V \rightarrow \mathbb{Z}_{+}$.

Saída: uma 2-partição conexa $\left(V_{1}, V_{2}\right)$ de $G$.

1. Encontre $A_{G}, H_{G}$ e construa $T_{G}$. Para cada $H \in H_{G}$ calcule $w_{H}$.

2. Para cada $H \in H_{G}$, seja $\left(V_{1}^{H}, V_{2}^{H}\right)$ a partição devolvida pelo algoritmo BalBicon 2 aplicado ao $\operatorname{par}\left(H, w_{H}\right)$.

3. Encontre $H_{0} \in H_{G}$ que maximiza $\min \left\{w_{H}\left(V_{1}^{H}\right), w_{H}\left(V_{2}^{H}\right)\right\}$ entre os blocos $H \in$ $H_{G}$, e reconstrua a partição conexa $\left(V_{1}, V_{2}\right)$ de $G$ tal que $V_{i}^{H_{0}}=V_{i} \cap H_{0}, i=1,2$.

4. Devolva $\left(V_{1}, V_{2}\right)$.

\section{fim_do_Algoritmo}

A seguir mostramos que o algoritmo $\mathrm{BalCon}_{2}$ preserva a razão de aproximação do algoritmo BalBicon 2 .

Teorema 3.3.3. Seja I uma instância do $\mathrm{PCB}_{2}$ que consiste de um grafo conexo $G=(V, E)$ e uma função $w: V \rightarrow \mathbb{Z}_{+}$. Então o algoritmo $\mathrm{BalCon}_{2}$, aplicado à instância $I$, devolve em tempo polinomial uma 2-partição conexa de $G$ com medida 
$m$, tal que

$$
\operatorname{opt}(I) \leq \frac{4}{3} m
$$

Prova: Para cada bloco $H$ de $G$, seja $m_{H}$ a medida da partição conexa de $H$ devolvida pelo algoritmo BalBicon 2 aplicado ao par $\left(H, w_{H}\right)$, e seja $\operatorname{opt}_{H}$ a medida de uma partição conexa ótima de $\left(H, w_{H}\right)$. Pelo Corolário 3.3.1, temos que opt $_{H} \leq 4 / 3 m_{H}$.

Seja $\left(V_{1}^{*}, V_{2}^{*}\right)$ uma solução ótima para a intância $I$. Pelo Lema 3.3.3, existe um bloco $H^{*}=\left(V^{*}, E^{*}\right)$ de $G$ tal que $\left(V_{1}^{*} \cap V^{*}, V_{2}^{*} \cap V^{*}\right)$ é uma partição conexa de $H^{*}$. Pela definição de $w_{H}$, temos que opt $(I)=\min \left\{w\left(V_{1}^{*} \cap V^{*}\right), w\left(V_{2}^{*} \cap V^{*}\right)\right\}=\operatorname{opt}_{H^{*}}$. Então, se $H_{0}$ é o bloco escolhido pelo algoritmo no passo 3 , temos que $m=m_{H_{0}} \geq$ $m_{H^{*}}$. Portanto,

$$
\operatorname{opt}(I)=\mathrm{opt}_{H^{*}} \leq \frac{4}{3} m_{H^{*}} \leq \frac{4}{3} m_{H_{0}}=\frac{4}{3} m
$$

Corolário 3.3.2. O algoritmo $\mathrm{BalCon}_{2}$ é uma 4/3-aproximação para o $\mathrm{PCB}_{2}$.

Prova: O resultado sobre a razão de aproximação segue do Teorema 3.3.3.

Vale a pena ressaltar que, se no passo 2 do algoritmo $\mathrm{BalCon}_{2}$ substituirmos o BalBicon 2 por um outro algoritmo (para grafos 2-conexos) com razão de aproximação $\alpha$, então o algoritmo que se obtém (para grafos conexos) também terá a mesma razão de aproximação $\alpha$. Nesse sentido, o estudo de algoritmos para o $\mathrm{PCB}_{2}$, no caso geral, pode ser concentrado no estudo de algoritmos para o caso de grafos 2-conexos. Essa observação, cuja prova segue da prova do Teorema 3.3.3, pode ser assim enunciada.

Corolário 3.3.3. Se existe uma $\alpha$-aproximação para o $\mathrm{PCB}_{2}$ restrito a grafos 2 -conexos, então existe uma $\alpha$-aproximação para o $\mathrm{PCB}_{2}$ (para grafos conexos).

Parece natural que, para grafos com conectividade mais alta, seja possível obter algoritmos com razões de aproximação melhores (do que aqueles apresentados até o momento). No entanto, não é muito óbvio como fazer uso dessa propriedade, e projetar tais algoritmos (se é que existem). 
Claramente, para resolver o $\mathrm{PCB}_{2}$ para grafos 3-conexos, podemos usar o algoritmo BalBicon2 (que aceita como entrada grafos 2-conexos). Porém, o BalBicon 2 essencialmente faz uso do fato de que os grafos 2-conexos têm a propriedade mencionada no Lema 3.3.1 (existência dos tais 2 vértices admissíveis). Claramente, no algoritmo BalBicon 2 se no momento da escolha de um vértice admissível (veja passo 4.1) pudéssemos garantir que há mais do que 3 deles, a razão de aproximação do algoritmo poderia ser melhorada. Basta notar que na análise que fizemos, usamos o fato de que o último vértice admissível testado tem peso menor ou igual ao peso de $v_{3}$ (isto porque tal vértice pode ser o próprio vértice $v_{3}$ ). Se houvesse, digamos $k \geq 4$ vértices admissíveis, poderíamos garantir que o peso desse último vértice admissível seria pelo menos o peso de $v_{k+1}$, e com isso, pelo Lema 3.3.2 teríamos que $t:=w(V) / w\left(v_{k+1}\right)$ seria pelo menos $k+1$. Assim, pelo Teorema 3.3.1 teríamos que $\operatorname{opt}(I) \leq(k+1) / k$; ou seja, a razão seria no máximo $5 / 4$.

Assim, a pergunta natural que surge é se o resultado mencionado no Lema 3.3.1 pode ser melhorado quando o grafo é, por exemplo, 3-conexo. Ou seja, se poderíamos garantir que, dada uma 2-partição conexa $\left(V_{1}, V_{2}\right)$ com $\left|V_{2}\right| \geq 3$, existem em $V_{2}$ pelo menos 3 vértices admissíveis. Infelizmente, isto não ocorre. $\mathrm{E}$ portanto, mesmo aumentando a conectividade do grafo de entrada, a análise (do desempenho) que fizemos não dá para ser melhorada usando esta abordagem. Isto sugere que para explorar o fato de que o grafo de entrada tem uma conectividade mais alta, precisamos introduzir outras idéias. De todo modo, vale ressaltar que se o grafo tem conectividade mais alta, não seria surpresa o algoritmo BalBicon $_{2}$ apresentar desempenho melhor do que a garantida razão de 4/3.

Antes de prosseguirmos, exibimos na Figura 3.4 uma instância $I$ para mostrar que a razão provada para o algoritmo BalBicon $_{2}$ no Teorema 3.3 .1 é justa mesmo para grafos 3-conexos. Observe que $w(V)=20, \beta=10$, opt $(I)=10$ e $t=5$.

Assim, seria desejável ter algoritmos para o $\mathrm{PCB}_{2}$ restrito a grafos 3-conexos, para os quais pudéssemos garantir desempenhos melhores do que o garantido pelo BalBicon $_{2}$. Nossas investigações nesta direção levaram a um algoritmo para grafos 3 -conexos que explora a seguinte propriedade de tais grafos: se o grafo tem pelo menos 4 vértices, sempre existe uma aresta que pode ser contraída e que deixa o novo grafo ainda 3-conexo. Antes de prosseguir, definimos o conceito de contração de arestas, e mencionamos o resultado no qual se baseia a estratégia do algoritmo que veremos a seguir. 


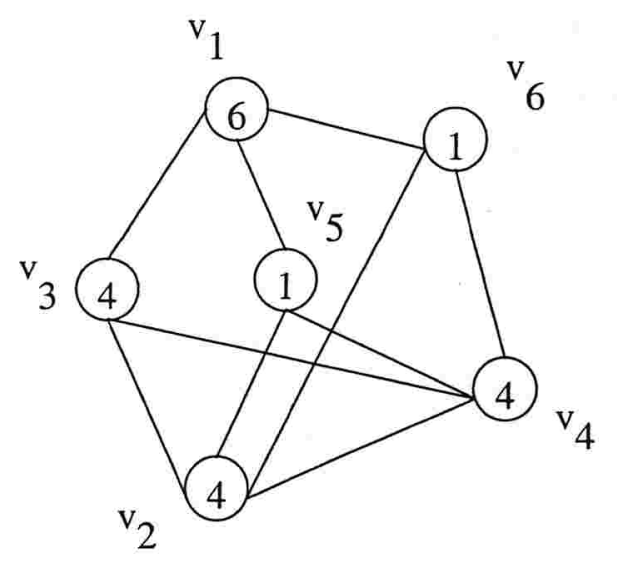

Figura 3.4: Grafo 3-conexo com pesos nos vértices.

Definição 3.3.3. Seja $e=u v$ uma aresta de um grafo $G=(V, E)$. Por $G /$ e denotamos o grafo obtido de $G$ pela contração da aresta e. Este grafo tem como conjunto de vértices o conjunto $V \backslash\{u, v\} \cup\left\{v_{e}\right\}$, onde $v_{e}$ é um novo vértice, e como conjunto de arestas $(E \backslash\{f \mid f$ adjacente a $u$ ou $v\}) \cup\left\{v_{e} y \mid y \in\right.$ $V \backslash\{u, v\}$ e y é adjacente a u ou $v$ em $G\}$. Ou seja, $G /$ e é obtido de $G$ removendose os vértices $u$ e $v$, e substituindo-os por um novo vértice, o qual se torna adjacente a todos os vértices y em $V \backslash\{u, v\}$ que eram vizinhos de $u$ ou $v$ em $G$. Neste caso, dizemos que G/e foi obtido pela contração da aresta e.

A prova do seguinte resultado pode ser encontrada em vários textos sobre grafos, e por isso será omitida (veja Diestel [Die00]).

Lema 3.3.5. Se $G$ é um grafo 3-conexo e $|V|>4$, então $G$ tem uma aresta e tal que $G /$ e é 3-conexo.

Definição 3.3.4. Se G é um grafo 3-conexo e e é uma aresta tal que G/e é 3conexo, então dizemos que e é uma aresta contraível.

No algoritmo que apresentamos a seguir, faremos contrações de arestas contraíveis objetivando encontrar arestas boas. Estas são definidas como segue.

Definição 3.3.5. Seja $G=(V, E)$ um grafo e $w: V \rightarrow \mathbb{Z}_{+}$. Definimos o peso de uma aresta $e=u v$, denotado por $w_{a}(e)$, como sendo a soma dos pesos dos vértices que são os seus extremos. Ou seja, $w_{a}(e)=w(u)+w(v)$. Dizemos que uma aresta e em $G$ é boa se $(2 / 5) w(V) \leq w_{a}(e) \leq(3 / 5) w(V)$. 
Algoritmo $\operatorname{Part3Con}(G, w)$

Entrada: um grafo 3-conexo $G=(V, E)$ e $w: V \rightarrow \mathbb{Z}_{+}$.

Saída: uma 2-partição conexa $\left(V_{1}, V_{2}\right)$ de $G$ e um conjunto $Z \subseteq V$.

${ }^{*}$ Através de $Z$ teremos controle sobre a qualidade da solução encontrada */

1. Seja $v_{1}=\arg \max \{w(v) \mid v \in V\}$ e $Z=\emptyset$.

2. Se $w\left(v_{1}\right) \geq(1 / 2) w(V)$ então

faça $V_{1}=\left\{v_{1}\right\}$ e $V_{2}=\bar{V}_{1}$; devolva $\left(V_{1}, V_{2}\right)$ e $Z$; pare.

3. Se $w\left(v_{3}\right) \leq(1 / 5) w(V)$ então

faça $\left(V_{1}, V_{2}\right)=\operatorname{BalBicon}_{2}(G, w)$; devolva $\left(V_{1}, V_{2}\right)$ e $Z$; pare.

4. Se existe vértice $v$ tal que $w(v) \geq(2 / 5) w(V)$ então

faça $V_{1}=\{v\}$ e $V_{2}=\bar{V}_{1}$; devolva $\left(V_{1}, V_{2}\right)$ e $Z$; pare.

5. Se existe aresta $u v$ boa então

faça $V_{1}=\{u, v\}$ e $V_{2}=\bar{V}_{1}$; devolva $\left(V_{1}, V_{2}\right)$ e $Z$; pare.

6. Seja $\tilde{G}=G ; \tilde{V}=V ; X=\emptyset . \quad I^{*} \operatorname{Vamos}$ procurar uma partição $(X, \bar{X}) * /$

7. Enquanto $|\tilde{V}|>4$ ou existe aresta $e$ contraível tal que $w_{a}(e)<(2 / 5) w(V)$ faça

7.1. $\tilde{G}=\tilde{G} / e ; \quad$ seja $v_{e}$ o novo vértice que substitui $e=u v$.

7.2. Faça $w\left(v_{e}\right)=w(u)+w(v)$.

7.3. Se existe aresta $x y$ boa então se $w(x)+w(y)>w(X)$ então $X=\{x, y\}$.

8. Se $|\tilde{V}|=4$ e existe uma aresta $u v$ boa em $\tilde{G}$ então faça $X=\{u, v\}$;

9. senão faça $(Z, \bar{Z})=$ BalBicon $_{2}(G, w)$.

10. Se $\min \{w(Z), w(\bar{Z})\} \geq \min \{w(X), w(\bar{X})\}$ então faça $\left(V_{1}, V_{2}\right)=(Z, \bar{Z})$;

11. senão reconstrua a partir de $(X, \bar{X})$ uma partição $\left(V_{1}, V_{2}\right)$ para $G$ e faça $Z=\emptyset$.

12. Devolva $\left(V_{1}, V_{2}\right)$ e $Z$.

\section{fim_do_Algoritmo}

Antes de analisarmos a razão de aproximação do algoritmo Part3Con, necessitamos do seguinte resultado. 
Lema 3.3.6. Seja $G=(V, E)$ um grafo 3 -conexo e $w: V \rightarrow \mathbb{Z}_{+}$. Seja $p$ um inteiro, $p \geq 1, G_{1}=G$ e para $i=1, \ldots, p$ seja $G_{i+1}=G_{i} / e_{i}$, onde e é uma aresta contraível de $G_{i}$. Suponha que, após a contração de cada aresta e $=x y$, o peso do novo vértice $v_{e}$ é definido como $w\left(v_{e}\right)=w(x)+w(y)$, e que os demais vértices mantêm o seu peso. Se $Q_{p}=\left(\tilde{V}_{1}, \ldots, \tilde{V}_{q}\right)$ é uma q-partição conexa de $G_{p}$, então esta partição induz uma q-partição conexa $\left(V_{1}, \ldots, V_{q}\right)$ de $G$ tal que $w\left(\tilde{V}_{i}\right)=w\left(V_{i}\right)$ para $i=1, \ldots, q$.

Prova: Faremos por indução em $p$. Para o caso $p=1$, considere $G_{1}=G / e_{1}$, e suponha que $e=e_{1}=x y$. Seja $\left(\tilde{V}_{1}, \ldots, \tilde{V}_{q}\right)$ uma $q$-partição conexa de $G_{1} \mathrm{e}$ suponha, sem perda de generalidade, que $v_{e} \in \tilde{V}_{1}$. Podemos obter uma $q$-partição conexa $\left(V_{1}, \ldots, V_{q}\right)$ de $G$ tomando $V_{i}=\tilde{V}_{i}$ para $i=2, \ldots, q$ e $V_{1}=\left(\tilde{V}_{1} \backslash\left\{v_{e}\right\}\right) \cup$ $\{x, y\}$. Claramente esta $q$-partição tem a propriedade desejada.

Suponhamos que a afirmação seja válida quando fazemos até $p-1$ contrações, $p \geq 2$. Vamos provar que ela continua válida quando fazemos $p$ contrações. Considere o grafo $G_{p}=G_{p-1} / e_{p-1}$. Pelo que foi demonstrado para $p=1$, dada uma $q$-partição conexa $Q_{p}=\left(\tilde{V}_{1}, \ldots, \tilde{V}_{q}\right)$ de $G_{p}$, sabemos construir uma $q$-partição conexa $Q_{p-1}$ de $G_{p-1}$. Agora, pela nossa hipótese indutiva, a partir de $Q_{p-1}$ podemos obter uma $q$-partição conexa $Q=\left(V_{1}, \ldots, V_{q}\right)$ para o grafo original $G$ tal que $Q$ tem a propriedade desejada.

Teorema 3.3.4. Seja I uma instância do $\mathrm{PCB}_{2}$ que consiste de um grafo 3-conexo $G=(V, E)$ e uma função $w: V \rightarrow \mathbb{Z}_{+}$. Sejam $\left(V_{1}, V_{2}\right)$ e $Z$, a partição e o conjunto, devolvidos pelo algoritmo Part3Con, aplicado à instância $I$. Então, $\left(V_{1}, V_{2}\right)$ é uma 2-partição conexa de $G$, e sua medida $m$ é tal que:

$$
\frac{\operatorname{opt}(I)}{m} \leq \frac{5}{4}, \text { se } Z=\emptyset ; \text { e } \frac{\operatorname{opt}(I)}{m} \leq \frac{4}{3} \text {, se } Z \neq \emptyset .
$$

Ademais, o algoritmo Part3Con pode ser implementado de modo que ele fique com complexidade polinomial.

Prova: Seja $P=\left(V_{1}, V_{2}\right)$ a partição devolvida pelo algoritmo. Vamos provar inicialmente que $P$ é uma partição conexa de $G$. É imediato que se $P$ é a solução devolvida nos passos $2,3,4$ e 5 então a afirmação é verdadeira. Se $P$ resulta da reconstrução mencionada no passo 11 , basta notar que $(X, \bar{X})$ é uma partição conexa (determinada no passo 7.3 ou no passo 8 ), e neste caso, pelo Lema 3.3.6 o 
resultado segue. Observe que no passo 7.1 o grafo $\tilde{G}$ é 3-conexo (pelo Lema 3.3.5). E se $P$ corresponde à partição $(Z, \bar{Z})$ obtida no passo 9 , o resultado segue do Teorema 3.3.1.

Vamos agora analisar a razão de aproximação. Note que se $Z=\emptyset$, então temos um dos 5 casos possíveis:

(1) $P$ foi devolvida no passo 2. Neste caso, $\operatorname{opt}(I)=m$.

(2) $P$ foi devolvida no passo 3. Neste caso, pelo Teorema 3.3.1, opt $(I) \leq t /(t-1)$ e $t \geq 5$. Logo, opt $(I) \leq 5 / 4 m$

(3) $P$ foi devolvida no passo 4. Então $m=w\left(V_{1}\right) \geq(2 / 5) w(V)$ e portanto, $\operatorname{opt}(I) \leq \frac{1}{2} w(V) \leq \frac{5}{4} m$.

(4) $P$ foi devolvida no passo 5. Então $m=w\left(V_{1}\right) \geq(2 / 5) w(V)$ e o resultado segue como no caso anterior.

(5) $P$ foi devolvida no passo 11, e corresponde à partição que foi obtida da partição $(X, \bar{X})$ de $\tilde{G}$ (construída no passo 7.3 ou passo 8). Neste caso, $w(X)$ tem o peso de uma aresta boa de $\tilde{G}$. Portanto, $2 / 5 W(V) \leq w\left(V_{1}\right) \leq$ $3 / 5 w(V)$, donde segue que $m=w\left(V_{1}\right) \geq(2 / 5) w(V)$. O resto da prova é análogo ao caso 3 .

Se $Z \neq \emptyset$ então a partição $P$ corresponde à partição $(Z, \bar{Z})$ construída no passo 9. Neste caso, pelo Corolário 3.3.2 temos que opt $(I) \leq 4 / 3 \mathrm{~m}$. Com isso, a prova está completa.

O grafo 3-conexo que exibimos na Figura 3.5 mostra que existem instâncias para as quais o algoritmo Part3Con pode obter uma solução melhor do que aquela produzida pelo algoritmo BalBicon .

Para essa instância $I$, o algoritmo BalBicon $_{2}$ faz iterações como descrito na Tabela 3.4. Note que $w(V)=32, \beta=16$ e opt $(I)=16$. Neste caso, o algortimo BalBicon $_{2}$ devolve a 2-partição conexa $V_{1}=\left\{v_{1}, v_{7}, v_{8}, v_{6}\right\}$ e $V_{2}=V \backslash V_{1}$ cuja medida é 4/3; e o algoritmo Part3Con devolve $V_{1}=\left\{v_{1}, v_{3}\right\}$ e $V_{2}=V \backslash V_{1}$, que é uma solução ótima. Note que $v_{1} v_{3}$ é uma aresta boa.

Na próxima seção exibimos um PTAS para o $\mathrm{PCB}_{2}$ restrito a uma classe especial de grafos. 


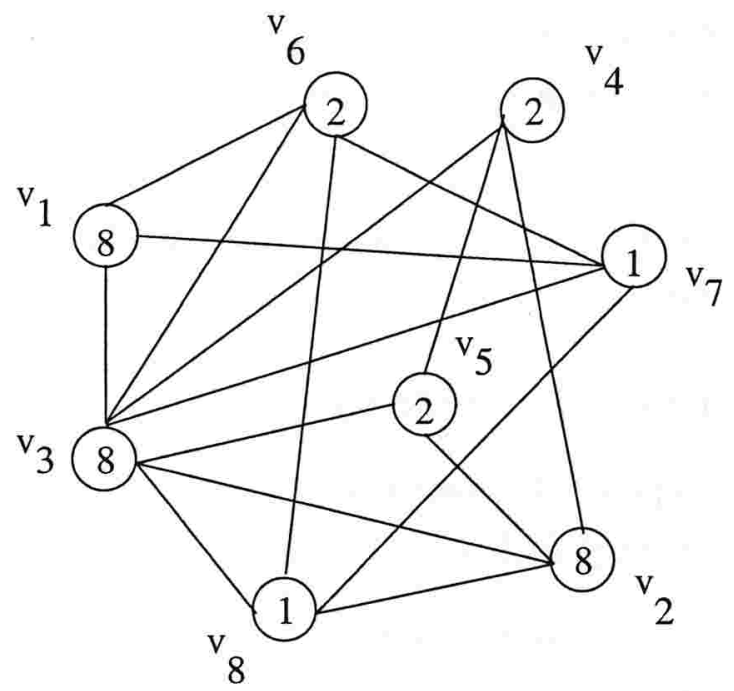

Figura 3.5: Um exemplo comparativo.

\begin{tabular}{|c|c|c|}
\hline \hline Iteração & $V_{1}$ & $u$ \\
\hline \hline 1 & $v_{1}$ & $v_{7}$ \\
\hline 2 & $v_{1}, v_{7}$ & $v_{8}$ \\
\hline 3 & $v_{1}, v_{7}, v_{8}$ & $v_{6}$ \\
\hline 4 & $v_{1}, v_{7}, v_{8}, v_{6}$ & $v_{3}$ \\
\hline
\end{tabular}

Tabela 3.4: Iterações executadas pelo algoritmo BalBicon $_{2}$.

\subsubsection{Esquema de Aproximação Polinomial}

Para descrevermos os resultados a respeito do esquema de aproximação polinomial, apresentaremos uma outra maneira de formular o problema Max 2-Partição Conexa Balanceada. Nesta definição (equivalente) a conexidade dos conjuntos da partição será substituída pela existência de uma árvore geradora em cada parte.

Antes de exibir a nova formulação, precisamos estabelecer a notação a ser usada.

Para isto, no que segue, considere que $G=(V, E)$ é um grafo com uma função $w: V \rightarrow \mathbb{Z}_{+}$, e que $W:=w(V)$.

Convenção 3.3.1. Se $T_{1}$ e $T_{2}$ são árvores que resultam de uma árvore geradora $T$ de $G$ após a remoção de uma aresta e de $T$, denotamos por $w\left(T_{1}\right)$ e $w\left(T_{2}\right)$ a soma dos pesos dos vértices em $T_{1}$ e $T_{2}$, respectivamente. Neste caso, $w\left(T_{1}\right) \leq\left\lfloor\frac{W}{2}\right\rfloor e$ 
$w\left(T_{2}\right) \geq\left\lceil\frac{W}{2}\right\rceil$, ou vice-versa. Por convenção, consideramos $w\left(T_{1}\right) \leq\left\lfloor\frac{W}{2}\right\rfloor$, isto é, $T_{1}$ é a árvore 'mais leve' de $T-e$.

Dado um grafo $G$, o $\mathrm{PCB}_{2}$ pode ser assim formulado: encontrar em $G$ uma árvore geradora $T$ que maximiza $m(G, T)=\max _{e \in E(T)} f(e, T)$, onde $f(e, T)=$ $w\left(T_{1}\right)$. A medida $m(G, T)$ é também chamada de balanceamento de $T$ e será denotada por Balance $(T)$.

$\mathrm{Na}$ tentativa de determinar a existência de um PTAS para o $\mathrm{PCB}_{2}$ estudamos o problema Max Mochila (veja definição no Apêndice).

Destacamos que os esquemas de aproximação polinomial para Max Mochila e para o $\mathrm{PCB}_{2}$ serão discutidos utilizando como medida o erro relativo (veja Definição 2.4.2). Como temos utilizado preferencialmente a razão de aproximação (veja Definição 2.3.2) para estimar a qualidade das soluções computadas, observamos que para problemas de maximização temos que $E(x, y)=1-\frac{1}{R(x, y)}$. Conseqüentemente, se $E(x, y) \leq \epsilon$ para algum $0<\epsilon<1$, então $R(x, y) \leq \frac{1}{1-\epsilon}$. Logo, resultados de aproximação em termos de erros relativos podem ser traduzidos para resultados em termos da razão de aproximação e vice-versa.

Um breve histórico de alguns resultados relacionados ao problema Max Mochila são comentados a seguir. Inicialmente, abordaremos um algoritmo [Hoc97] pseudopolinomial baseado em programação dinâmica que resolve o Max 0/1 Mochila, cuja formulação como um programa linear inteiro é a seguinte:

$$
\begin{aligned}
& P^{*}=\operatorname{Max} \sum_{j=1}^{n} p_{j} x_{j} \\
& \text { sujeito a } \sum_{j=1}^{n} a_{j} x_{j} \leq B \\
& \qquad x_{j} \in\{0,1\} \text {, para } j=1, \ldots, n .
\end{aligned}
$$

Seja $F_{j}(i)$ o menor volume da 'mochila' que produz um valor $i$ da função objetivo envolvendo variáveis no conjunto $\{1, \ldots, j\}$. As condições de contorno são

$$
F_{j}(0)=0, j=1, \ldots, n,
$$

e a fórmula recursiva é

$$
F_{j}(i)=\min \left\{F_{j-1}\left(i-p_{j}\right)+a_{j}, F_{j-1}(i)\right\} .
$$

Usando as condições de contorno, a tabela de valores é avaliada em ordem crescente dos valores da função objetivo:

$$
F_{1}(1), F_{2}(1), \ldots, F_{n}(1) ; F_{1}(2), \ldots, F_{n}(2) ; \ldots
$$


A computação termina quando o maior valor de $i$ é descoberto tal que $F_{n}(i) \leq B$.

Cada função é avaliada em $O(1)$ passos, e existe um total de $O\left(n P^{*}\right)$ avaliações de funções, onde $P^{*}$ é o valor ótimo da função objetivo. Assim, o tempo de execução deste algoritmo baseado em programação dinâmica é $O\left(n P^{*}\right)$.

A idéia do FPTAS é explorar o algoritmo descrito acima, mas normalizando os coeficientes da função objetivo. Isto acarreta no tempo de execução do algoritmo uma dependência com o novo valor normalizado da solução ótima. Por outro lado, para uma escolha cuidadosa do valor de normalização, a função objetivo do problema normalizado é próxima daquela do problema original.

Considere a normalização dos coeficientes da função objetivo por um fator $k$. Os coeficientes normalizados são então $p_{j}(k)=\left\lfloor\frac{p_{j}}{k}\right\rfloor$, e o problema normalizado,

$$
\begin{aligned}
& \quad \operatorname{Max} \sum_{j=1}^{n} p_{j}(k) x_{j} \\
& \text { sujeito } a \sum_{j=1}^{n} a_{j} x_{j} \leq B \\
& \qquad x_{j} \in\{0,1\}, \text { para } j=1, \ldots, n .
\end{aligned}
$$

O tempo de execução exigido para resolver o problema normalizado depende do valor do ótimo. Como o valor da solução ótima é reduzido por um fator $k$, isto também ocorre com o tempo de execução.

Seja $P_{\text {max }}=\max _{j=1, \ldots, n}\left\{p_{j}\right\}$. Observe então que

$$
P_{\max } \leq P^{*} \leq n P_{\max }
$$

Com este limite superior, o tempo de execução do algoritmo baseado em programação dinâmica para resolver otimamente o problema normalizado é $O\left(n^{2}\left\lfloor\frac{P_{\max }}{k}\right\rfloor\right)$.

Seja $S^{*}$ o conjunto de índices das variáveis na solução ótima do problema Max 0/1 Mochila, e $S(k)$ o conjunto de índices das variáveis na solução ótima do problema normalizado. Então

$$
\sum_{j \in S(k)} p_{j} \geq \sum_{j \in S(k)} k\left\lfloor\frac{p_{j}}{k}\right\rfloor \geq \sum_{j \in S^{*}} k\left\lfloor\frac{p_{j}}{k}\right\rfloor \geq \sum_{j \in S^{*}}\left(p_{j}-k\right) \geq \sum_{j \in S^{*}} p_{j}-k\left|S^{*}\right| .
$$

Logo, o erro absoluto (veja Definição 2.4.1) da solução normalizada é no máximo $k\left|S^{*}\right|$, e o erro relativo, usando o limite inferior (1) é

$$
\epsilon^{\prime}=\frac{k\left|S^{*}\right|}{P_{\max }} \leq n \frac{k}{P_{\max }}
$$


O tempo de execução é $O\left(n^{2} \frac{P_{\max }}{k}\right)=O\left(n^{3} \frac{1}{\epsilon^{\prime}}\right)$. Temos então um FPTAS.

Ibarra e Kim [IK75] apresentaram alguns refinamentos do FPTAS mencionado envolvendo outros limites sobre o ótimo e a separação dos itens em grandes e pequenos. Os itens grandes são aqueles com valores $p_{j}$ maiores ou iguais a um valor limite $T$, e os pequenos têm estes valores menores do que $T$. Esses autores provaram que a soma dos erros da solução dos itens grandes com aquela dos itens pequenos é um limite superior para o erro total.

Para obter um limite melhor, considere os índices das variáveis em Max 0/1 Mochila organizados em ordem decrescente da razão $\frac{p_{j}}{a_{j}}$. Seja $\bar{j}$ o maior índice tal que $\sum_{j=1}^{\bar{j}} a_{j} \leq B$ e seja $P_{0}=\max \left\{P_{\max }, \sum_{j=1}^{\bar{j}} p_{j}\right\}$. Então $P_{0} \leq P^{*} \leq 2 P_{0}$ (a prova da validade destes limites pode ser encontrada em [IK75, Law79]).

Para um valor escolhido de $\epsilon^{\prime}$, Lawler [Law79] define os itens grandes como aqueles com $p_{j} \geq T=\frac{1}{2} \epsilon^{\prime} P_{0}$, e os itens pequenos como sendo todos os demais. $\mathrm{O}$ fator normalizador selecionado é $k=\frac{1}{4} \epsilon^{2} P_{0}$. O tempo de execução obtido é atingido executando-se a fase de programação dinâmica apenas para os itens grandes. Os itens pequenos são 'empacotados' na ordem descrescente da razão $\frac{p_{j}}{a_{j}}$ enquanto ainda existe folga na restrição de empacotamento. O erro total resultante da união destes dois conjuntos de variáveis selecionadas é no máximo $\epsilon^{\prime}$. Para verificar que o erro total não excede $\epsilon^{\prime}$, note que o erro absoluto obtido da solução com programação dinâmica sobre os itens grandes não excede $k \frac{P^{*}}{T}$ quando o número de itens grandes na solução ótima do problema normalizado é $\frac{P^{*}}{T}$. Para os itens pequenos, o erro absoluto não excede o valor $T$. A soma dos erros relativos é então,

$$
\frac{k}{T}+\frac{T}{P^{*}} \leq \frac{\frac{1}{4} \epsilon^{\prime 2} P_{0}}{\frac{1}{2} \epsilon^{\prime} P_{0}}+\frac{\frac{1}{2} \epsilon^{\prime} P_{0}}{P_{0}}=\epsilon^{\prime} .
$$

A vantagem computacional é obtida do número relativamente pequeno de itens grandes que precisam ser considerados. O número de valores diferentes de $p_{j}(k)$ é limitado por $\frac{P^{*}}{k}$. Usando o fato de que $P^{*} \leq 2 P_{0}$, temos que o número de valores diferentes é limitado por $\frac{8}{\epsilon^{\prime 2}}$. Como para cada valor de $p_{j}(k)$, poderia existir no máximo $n_{j}=\left\lfloor\frac{p^{*} / k}{p_{j}(k)}\right\rfloor$ itens de tamanho $p_{j}(k)$ em uma solução ótima, e entre todos aqueles com o mesmo valor $p_{j}(k)$, aqueles com os menores pesos seriam selecionados, outros itens grandes não precisam ser considerados pois são dominados. Com este tipo de argumento, Lawler provou que o número de itens grandes considerados é $\frac{6}{\epsilon^{\prime 2}} \log \frac{4}{\epsilon^{\prime}}$. Conseqüentemente, o tempo de execução obtido para a fase de programação dinâmica sobre os itens grandes é $O\left(\frac{n P_{0}}{k}\right)=O\left(\frac{1}{\epsilon^{4}} \log \frac{1}{\epsilon^{\prime}}\right)$. 
Lawler também mostrou que existe um limite mais justo de $O\left(\frac{1}{\epsilon^{4 / 4}}\right)$ sobre o número de itens grandes resultando em um tempo de execução de $O\left(\frac{1}{\epsilon^{14}}\right)$.

Resumindo, os passos do algoritmo de aproximação $\mathrm{A}_{\epsilon^{\prime}}$, para Max 0/1 Mochila são:

1. Descobrir o valor e o conjunto de elementos correspondentes a $P_{0}$.

2. Descobrir os itens grandes que são candidatos a serem incluídos na solução ótima.

3. Resolver o problema normalizado para os itens grandes usando programação dinâmica.

4. Descobrir a maior quantidade de itens pequenos que pode ser empacotada no volume restante da mochila.

Os passos 1 e 2 podem ser executados em tempo linear, o passo 4 em $O\left(n \log \frac{1}{\epsilon^{\prime}}\right)$, e o passo $3 \mathrm{em} O\left(\frac{1}{\epsilon^{\prime 4}}\right)$. Logo, o tempo de execução total é $O\left(n \log \frac{1}{\epsilon^{\prime}}+\frac{1}{\epsilon^{14}}\right)$.

Baseando-nos nas idéias discutidas anteriormente, desenvolvemos um PTAS para o $\mathrm{PCB}_{2}$ restrito a $\Gamma$-grafos onde $w(v)=1$ para todo vértice $v$ que não é um ponto de articulação. Na intenção de apresentar o esquema de aproximação para esta classe de grafos, começamos exibindo um PTAS para $C_{w}$-grafos, que denominamos Balance_2CGrafo. Lembramos que essas classes de grafos foram estudadas na Seção 3.1.1.

No algoritmo Balance_2CGrafo utilizamos idéias similares àquelas exibidas no algoritmo $\mathrm{A}_{\epsilon}$ para Max 0/1 Mochila. O fator de normalização usado é $\frac{1}{4} \epsilon^{2} \tilde{B}$ e o valor de $Q$, fator de separação dos itens pequenos e grandes é $\epsilon \tilde{B}$. Aqui $\tilde{B}$ possui a mesma função e propriedades de $P_{0}$ para Max 0/1 Mochila. Contudo o passo 1 , do esquema geral descrito acima, é executado diferentemente e baseado na idéia central do algoritmo MaxBal2, desenvolvido por Galbiati, Maffioli e Morzenti [GMM97]. A etapa de separação de itens pequenos e grandes é similar: no problema em questão os itens são os vértices do grafo e o que determina a magnitude de cada vértice é seu peso. Naturalmente, temos uma diferença crucial entre Max 0/1 Mochila e o $\mathrm{PCB}_{2}$ que é o requisito de conexidade dos vértices escolhidos, grandes e pequenos. Isto determina as diferenças mais relevantes dos passos 3 e 4 . Em ambas as etapas precisamos de um procedimento que verifica a conexidade da solução sendo testada. No passo 3 continuamos utilizando programação 
dinâmica, mas armazenamos, para cada valor da função objetivo obtido, todas as soluções viáveis que atingiram este valor. Posteriormente, escolhemos para cada valor obtido da função objetivo, aquela solução dos vértices grandes que admite uma soma de pesos dos vértices pequenos tal que o balanceamento obtido é o máximo possível, obviamente respeitando-se o critério de conexidade das partições geradas. Depois, devolvemos a árvore para a qual foi obtida a melhor solução viável. Mais formalmente, podemos descrever nossa estratégia como segue.

Algoritmo Balance_2CGrafo ${ }_{\epsilon}(G, w)$
$/^{*} \quad \epsilon$ é a aproximação desejada. */

Entrada: um $C_{w}$-grafo $G=(V, E)$, uma função $w: V \rightarrow \mathbb{Z}_{+}$e a aproximação exigida $\epsilon$. Saída: um par $(T, B)$ onde $T$ é uma árvore geradora de $G$ e $B=\operatorname{Balance}(T)=$ $\max _{e \in E(T)} f(e, T)$.

1. Seja $W=w(V)$ e Metade $=\lfloor W / 2\rfloor$.

2. Seja $T$ uma árvore geradora arbitrária de $G$.

3. Para cada aresta $e$ de $T$, seja $\alpha_{e}$ o peso da árvore 'mais leve' de $T-e$.

4. Seja $e^{\prime}$ a aresta de $T$ tal que $\alpha_{e^{\prime}}=\max _{e \in E(T)}\left\{\alpha_{e}\right\}$. Seja $T_{\alpha}$ e $T_{\beta}$ as árvores de $T-e^{\prime}$, onde $\alpha_{e^{\prime}}=w\left(T_{\alpha}\right)$. Seja $B=\alpha_{e^{\prime}}$.

5. Enquanto $B<$ Metade faça

(a) Seja $y$ o extremo de $e^{\prime}$ que pertence a $T_{\beta}$. Escolha uma aresta $e=t z$ tal que $t \in V\left(T_{\alpha}\right)$ e $z \in V\left(T_{\beta}\right), z \neq y$. Seja $e^{\prime \prime}=z p$, onde $p$ é o vértice adjacente a $z$ no caminho de $z$ até $y$ em $T_{\beta}$. Seja $B=B+\alpha_{e^{\prime \prime}}$.

(b) Se $B \leq$ Metade então seja $T=T+e-e^{\prime \prime}$.

6. Se $B=$ Metade então devolva $(T, B)$.

7. senão

(a) Seja $\widetilde{B}=B-\alpha_{e^{\prime \prime}}$, onde $\alpha_{e^{\prime \prime}}$ é o último valor adicionado a $B$ no passo 5 a. Seja $Q=\epsilon \widetilde{B}$.

(b) Sejam $S$ mall $=\{v \in V \mid w(v) \leq Q\}$, o conjunto de vértices pequenos; e Large $=\{v \in V \mid w(v)>Q\}$, o conjunto de vértices grandes.

(c) Se $S$ mall $=V$ então $(T, B)=\operatorname{Add} \_l e a v e s\left(T_{\alpha}, T_{\beta}, V\left(T_{\beta}\right)\right)$. Devolva $(T, B)$.

(d) senão

i. Seja CombLarge $=\{X \mid \emptyset \neq X \subseteq$ Large $\}$.

ii. Para cada $v \in$ Large, seja $q_{v}=\operatorname{Scale}(w(v))$. 
iii. Seja $C(j)=\left\{X \in\right.$ CombLarge $\left.\mid \sum_{v \in X} q_{v}=j\right\}$ e $r=\max \{j \mid C(j) \neq$ $\emptyset\}$.

iv. Para $j=0, \ldots, r$ faça

- Para cada $X \in C(j)$ tal que $w(X) \leq \operatorname{Metade~faça~Find-Tree~}(X, j)$.

v. Para $j=0, \ldots, r$ faça $T(j)=\operatorname{Best} \_\operatorname{Tree}(j)$.

vi. Se $S$ mall $\neq \emptyset$ então $T(r+1)=$ Small_Tree.

vii. Encontre $j(j=0, \ldots, r+1)$ tal que $T(j)$ é uma árvore com o maior balanceamento. Seja $T=T(j)$ e $B=\operatorname{Balance}(T)$. $\operatorname{Devolva}(T, B)$.

\section{fim_do_Algoritmo}

No que segue, descrevemos os procedimentos usados no algoritmo. Antes disto, lembramos que, dada uma aresta $e$ de uma árvore geradora $T$ de $G$, ao referenciarmos as duas árvores $T_{1}$ e $T_{2}$ de $T-e$ consideramos que $w\left(T_{1}\right) \leq$ METADE (veja Convenção 3.3.1).

- Add_leaves $\left(T_{1}, T_{2}, Y\right)$ : Move uma folha de $Y$ para $T_{1}$ substituindo arestas da árvore por arestas do grafo $G$ (não pertencentes à árvore) enquanto $w\left(T_{1}\right) \leq$ Metade. Se descobrimos uma folha $v$ em $Y$ adjacente a algum vértice de $T_{1}$ tal que $w\left(T_{1}\right)+w(v)>$ METADE, então paramos. A árvore geradora $T$ obtida após todas as operações de atualização e seu balanceamento $B$ são devolvidos.

Podemos supor que existe pelo menos um vértice de $Y$ (possivelmente o extremo da aresta conectando $T_{1}$ a $T_{2}$ ) que é uma folha de $T_{2}$ e é adjacente a algum vértice de $T_{1}$. Caso contrário, é possível trocar uma aresta do grafo $G$ (não pertencente à árvore) com uma aresta da árvore tal que ambas as árvores $T_{1}$ e $T_{2}$ mantêm os mesmos vértices mas uma folha em $T_{2}$ que pertence a $Y$ e é adjacente a um vértice de $T_{1}$ é criado. Isto é possível porque $G$ é 2-conexo.

- Scale $(x)$ : Calcula e devolve o valor escalonado $\left\lfloor\frac{x}{2^{s} k}\right\rfloor 2^{s}$ onde $k=\frac{1}{4} \epsilon^{2} \widetilde{B}$ e $s$ é tal que $x \in\left(2^{s} Q, 2^{s+1} Q\right]$.

- Find_Tree $(X, j)$ : Constrói uma árvore geradora $T$ de $G$ que tem uma aresta $e$ tal que a árvore $T_{1}$ de $T-e$ contém como vértices grandes somente o conjunto $X$, ou devolve $n u l l$.

Descrevemos anteriormente, a funcionalidade do procedimento Find_Tree. No que segue, explicamos como o executamos.

Para cada conjunto $C(j), 0 \leq j \leq r$, diferenciamos duas classes de árvores geradoras construídas por Find_Tree: 
1. aquelas árvores $T$ obtidas usando o procedimento Add_leaves ou com $\operatorname{Balance}(T)=\operatorname{Metade}$;

2. e as demais árvores restantes.

A árvore geradora que possui o maior balanceamento de cada classe é referenciada como $\operatorname{Best1}(j)$ e $\operatorname{Best} 2(j)$, respectivamente. Com este objetivo, precisamos analisar dois casos.

1. Small $=\emptyset$ : Neste caso, verificamos se $G[X]$ e $G[V \backslash X]$ são ambos conexos. Se isto ocorre então devolvemos uma árvore geradora $T$ que conecta $G[X]$ a $G[V \backslash X]$ usando uma única aresta $e$. Se $w\left(T_{1}\right)=\operatorname{METADE}$ então $\operatorname{Best} 1(j)=$ $T$, senão atualizamos $\operatorname{Best} 2(j)$, se for necessário.

2. Small $\neq \emptyset$ : Neste caso, o conjunto $X$ induz um clique pela nossa hipótese ${ }^{3}$. Seja $C_{1}$ o componente de $G-X$ com peso máximo. Se $w\left(C_{1}\right)<$ Metade então devolvemos null. Caso contrário, consideramos dois casos novamente.

(a) (Large $\backslash X) \subseteq C_{1}$ : Neste caso, adicionamos a $X$ tantos vértices pequenos quanto possível. Estes vértices são alocados como descrevemos a seguir. Seja $A=V \backslash V\left(C_{1}\right)$ e $X^{\prime}=$ Large $\backslash X$. Enquanto $w(A) \leq$ Metade e existe um componente $C^{\prime}$ de $\left(C_{1} \backslash X^{\prime}\right)$ com um vértice $u$ tal que $u v \in E$ para algum vértice $v \in A$, atualizamos o conjunto $A$ tal que $A=A \cup V\left(C^{\prime}\right)$. Quando isto não ocorre, consideramos $T$ uma árvore geradora que conecta $G[A]$ a $G[V \backslash A]$ usando uma única aresta $e^{\prime}$ e analisamos três situações que podem ocorrer.

i. $w(A)<$ Metade: Se a aresta $e^{\prime}$ de $T$ maximiza $f_{A B}(e, T)$ entre todas as arestas então devolvemos $T$, caso contrário devolvemos null. Também, atualizamos Best2(j), se necessário.

ii. $w(A)=$ METADE: Neste caso, devolvemos a árvore geradora $T$ e definimos $\operatorname{Best} 1(j)=T$.

iii. $w(A)+w\left(C^{\prime}\right)>$ Metade: Quando isto ocorre, chamamos Add_leaves $\left(T_{1}, T_{2}, C^{\prime}\right)$ onde $T_{1}$ e $T_{2}$ são árvores geradoras de $G[A]$ e $G[V \backslash A]$, respectivamente. Adicionalmente, devolvemos a árvore

\footnotetext{
${ }^{3}$ Neste caso, cada vértice $v \in V$ tal que $w(v)=1$ é pequeno e talvez isto pode ocorrer também com outros vértices com pesos diferentes.
} 
geradora $T$ obtida deste procedimento e atualizamos $\operatorname{Best} 1(j)$, se necessário.

(b) $X=$ Large: Sob esta condição, chamamos Add_leaves $\left(T_{1}, T_{2}, V\left(C_{1}\right)\right)$ onde $T_{1}$ e $T_{2}$ são árvores geradoras de $G\left[V \backslash V\left(C_{1}\right)\right]$ e $G\left[V\left(C_{1}\right)\right]$, respectivamente. Também devolvemos a árvore geradora $T$ obtida deste procedimento e atualizamos $\operatorname{Best} 1(j)$, se necessário.

- Small_Tree: Constrói uma árvore geradora $T$ de $G$ que possui uma aresta $e$ tal que a árvore $T_{1}$ de $T-e$ contém somente vértices pequenos e $w\left(T_{1}\right) \leq \operatorname{Balance}(T)$.

Encontramos uma árvore nas condições descritas anteriormente seguindo os passos descritos a seguir.

Seja $C_{1}$ um componente de $G$ - Large com peso máximo. Seja $T$ uma árvore geradora que conecta com uma única aresta o componente $C_{1}$ a $G\left[V \backslash V\left(C_{1}\right)\right]$. Se $w\left(C_{1}\right) \leq$ METADE então devolvemos $T$. Caso contrário, seja $e$ uma aresta de $T$ que maximiza $f(e, T)$ entre todas as arestas de $C_{1}$. Sejam $T_{1}$ e $T_{2}$ as duas árvores de $T-e$. Então, chamamos Add_leaves $\left(T_{1}, T_{2}, V\left(C_{1}\right)\right)$ e devolvemos a árvore geradora $T$ obtida com este procedimento.

- Best_Tree $(j)$ : Determina a melhor árvore geradora de cada coleção $C(j)$ para $0 \leq j \leq r$.

Observe que conseguimos a melhor árvore, apenas analisando a seguinte condição: se $\operatorname{Best1}(j) \neq$ null então devolvemos $\operatorname{Best} 1(j)$, senão devolvemos $\operatorname{Best2}(j)$.

Após discutirmos cada procedimento utilizado no algoritmo Balance_2CGrafo ${ }_{\epsilon}$, mostramos a seguir alguns resultados essenciais para demonstrarmos que temos de fato um esquema de aproximação polinomial.

Lema 3.3.7. Suponha que para a entrada $(G, w)$ o algoritmo Balance_2CGrafo ${ }_{\epsilon}$ executa o passo 7. Seja $\widetilde{B}$ o valor definido no passo 7a, e seja $B^{*}$ o balanceamento de uma solução ótima para essa entrada. Então, $\frac{W}{4} \leq \widetilde{B} \leq B^{*} \leq 2 \widetilde{B}$.

Prova: Considere o valor de $B$ depois da execução do passo 5 . Como $\widetilde{B}=$ $B-\alpha_{e^{\prime \prime}}$, onde $\alpha_{e^{\prime \prime}}$ é o último valor adicionado a $B$ no passo 5 a (veja passo 7 a), 
temos que $\widetilde{B}<\operatorname{Metade}<\widetilde{B}+\alpha_{e^{\prime \prime}}$. Assim, $\widetilde{B} \leq B^{*}<\widetilde{B}+\alpha_{e^{\prime \prime}} \leq \widetilde{B}+\alpha_{e^{\prime}} \leq 2 \widetilde{B}$, onde $\alpha_{e^{\prime}}$ é o valor definido no passo 4. Para deduzir a última inequação, note que $\alpha_{e^{\prime}}$ é precisamente o balanceamento da árvore inicial considerada no passo 2. Além disto, observe que depois das atualizações (se alguma ocorre) a árvore após o passo 5 tem balanceamento $\widetilde{B}$, e claramente, $\widetilde{B} \geq \alpha_{e^{\prime}}$. Visto que Metade $=\lfloor W / 2\rfloor<\widetilde{B}+\alpha_{e^{\prime \prime}} \leq 2 \widetilde{B}$, isto implica que $\frac{W}{4} \leq \widetilde{B}$.

Juntando as inequações obtidas, temos $\frac{W}{4} \leq \widetilde{B} \leq B^{*} \leq 2 \widetilde{B}$.

Utilizando argumentos similares àqueles de Lawler para Max 0/1 Mochila, determinamos que o número de vértices que devem ser considerados para a computação dos vértices grandes é $O\left(1 / \epsilon^{2}\right)$.

Lema 3.3.8. Seja $\epsilon$ um racional tal que $0<\epsilon<1$. Considere o algoritmo Balance_2CGrafo $\epsilon_{\epsilon}$ aplicado a uma instância $(G, w)$. Então o número de vértices grandes definido no passo $7 b$ é $O\left(\frac{1}{\epsilon^{2}}\right)$.

Prova: Seja $G=(V, E)$ onde $V=\left\{v_{1}, \ldots, v_{n}\right\}$ e $W=w(V)$. Seja $q_{i}=q_{v_{i}}$ para $1 \leq i \leq n$. Inicialmente, observe que o tamanho ${ }^{4}$ do espaço dos pesos escalonados $\left(q_{1}+\ldots+q_{n}\right)$ é limitado por

$$
\frac{W}{k}=\frac{W}{\frac{1}{4} \epsilon^{2} \widetilde{B}} \leq \frac{W}{\frac{1}{4} \epsilon^{2} \frac{W}{4}}=\frac{16}{\epsilon^{2}},
$$

onde a desigualdade é válida pelo Lema 3.3.7.

O número de valores $q_{i}$ distintos obtidos de cada $w\left(v_{i}\right)$-intervalo $\left(2^{s_{i}} Q, 2^{s_{i}+1} Q\right]$ é no máximo $\left(\frac{4}{\epsilon}+1\right)$, para qualquer $0 \leq s_{i} \leq\left\lfloor\log \left(\frac{W}{Q}\right)\right\rfloor$. Isto ocorre porque $q_{i}=$ $\left\lfloor\frac{w\left(v_{i}\right)}{2^{s_{i}}}\right\rfloor 2^{s_{i}}$, o que implica $k q_{i} \leq w\left(v_{i}\right)<k q_{i}+2^{s_{i}} k$. Assim, dois termos consecutivos são $\left\lfloor\frac{k q_{i}}{2^{s_{i} k}}\right\rfloor 2^{s_{i}}=\left\lfloor\frac{q_{i}}{\left.2^{s_{i}}\right\rfloor} 2^{s_{i}}\right.$ e $\left\lfloor\frac{k q_{i}+2^{s_{i} k}}{2^{s_{i} k}}\right\rfloor 2^{s_{i}}=\left\lfloor\frac{q_{i}}{2^{s_{i}}}+1\right\rfloor 2^{s_{i}}$. Como $\left\lfloor\frac{q_{i}}{2^{s_{i}}}+1\right\rfloor 2^{s_{i}}-\left\lfloor\frac{q_{i}}{\left.2^{s_{i}}\right\rfloor} 2^{s_{i}}=\right.$ $\left\lfloor\frac{q_{i}}{2^{s_{i}}}\right\rfloor 2^{s_{i}}+2^{s_{i}}-\left\lfloor\frac{q_{i}}{2^{s_{i}}}\right\rfloor 2^{s_{i}}=2^{s_{i}}$, temos que os valores $q_{i}$ distintos de cada $q_{i}$-intervalo produz uma progressão aritmética de razão $2^{s_{i}}$.

Além disto, o número de vértices grandes com peso escalonado $q_{i}$ é no máximo $n_{i}=\left\lfloor\frac{W}{k q_{i}}\right\rfloor$. Caso contrário, suponha que temos um conjunto $U$ de vértices grandes com peso escalonado $q_{i}$ tal que $|U| \geq n_{i}+1$. Assim, $\sum_{v \in U} q_{v}>\frac{W}{k}$ o que implica $\sum_{v \in U} \frac{w(v)}{k}>\frac{W}{k}$. Por definição de $W$, isto é uma contradição.

\footnotetext{
${ }^{4}$ Lembre-se que $q_{1}+\ldots+q_{n}<\frac{w\left(v_{1}\right)}{k}+\ldots+\frac{w\left(v_{n}\right)}{k}=\frac{W}{k}$.
} 
Para cada $q_{i}$-intervalo $(a, b]$, o valor médio de $n_{i}$ é por volta de $\left(\left(16 / \epsilon^{2} a\right)+\right.$ $\left.\left(16 / \epsilon^{2} b\right)\right) / 2$.

Então, para o intervalo escalonado $(4 / \epsilon, 8 / \epsilon]$ o número de valores $q_{i}$ é algo em torno de $\left(\frac{4}{\epsilon}+1\right)$ e o valor médio de $n_{i}$ é da ordem de $3 / \epsilon$. Para o intervalo $(8 / \epsilon, 16 / \epsilon]$ o número de valores $q_{i}$ é ainda em torno de $\left(\frac{4}{\epsilon}+1\right)$ e o valor médio de $n_{i}$ é da ordem de $3 / 2 \epsilon$. No intervalo $(16 / \epsilon, 32 / \epsilon]$ temos novamente por volta de $\left(\frac{4}{\epsilon}+1\right)$ valores de $q_{i}$ e o valor médio de $n_{i}$ em torno de $3 / 4 \epsilon$ and so on. Isto implica que o número de vértices grandes é limitado por $\left(\frac{4}{\epsilon}+1\right) \cdot \frac{3}{\epsilon}\left(1+\frac{1}{2}+\ldots+\frac{Q}{W}\right) \leq \frac{24}{\epsilon^{2}}+\frac{6}{\epsilon} \leq \frac{30}{\epsilon^{2}}$, onde a primeira inequação segue do fato que $\left(1+\frac{1}{2}+\ldots+\frac{Q}{W}\right)$ é uma progressão geométrica cuja soma é menor do que 2.

Isto acarreta ao algoritmo um tempo de execução limitado por alguma função polinomial no tamanho da instância e em $O\left(2^{O\left(1 / \epsilon^{2}\right)}\right)$. Logo, para $\epsilon$ fixo, temos que o tempo total é polinomial no tamanho da entrada.

Teorema 3.3.5. Seja I uma instância que consiste de um $C_{w}$-grafo $G=(V, E) e$ seja $\epsilon$ um racional, $0<\epsilon<1$. O algoritmo Balance_2C $\mathrm{Grafo}_{\epsilon}$ aplicado à instância I tem complexidade limitada por $q(|I|, \epsilon)$, onde $q$ é um polinômio, e devolve uma solução aproximada $T$ com balanceamento $B$ satisfazendo $\frac{B^{*}-B}{B^{*}} \leq \epsilon$, onde $B^{*}$ é o balanceamento de uma solução ótima.

Prova: (a) Complexidade: Seja $I$ a entrada do algoritmo Balance_2CGrafo ${ }_{\epsilon}$. Observe que os passos 1 até 6 podem ser executados em tempo polinomial em $|I|$. $\mathrm{O}$ passo 7 necessita de uma análise mais detalhada. Os passos $7 \mathrm{a}$ e $7 \mathrm{~b}$ podem ser facilmente executados. O passo 7c depende do procedimento Add_leaves, que pode ser executado em tempo polinomial em $|I|$. O passo $7(\mathrm{~d})$ i gasta tempo $2^{O\left(1 / \epsilon^{2}\right)}$, visto que $\mid$ Large $\mid=O\left(1 / \epsilon^{2}\right)$ pelo Lema 3.3.8. O passo $7(\mathrm{~d})$ ii efetua $O\left(1 / \epsilon^{2}\right)$ chamadas do procedimento Scale, que pode ser executado em tempo polinomial em $|I|$. O passo 7 (d)iii calcula a soma $\sum_{v \in X} q_{v}$ para cada conjunto $X \in$ CombLarge. Então, gasta um tempo da ordem de $2^{O\left(1 / \epsilon^{2}\right)} * O(|I|)$. O passo 7 (d)iv é executado não mais do que $2^{O\left(1 / \epsilon^{2}\right)}$, cada vez com a execução do procedimento Find_Tree que pode ser executado em tempo polinomial em $|I|$. Observe que $r$ é limitado por $16 / \epsilon^{2}$ (veja Lema 3.3.8). Assim, o passo $7(\mathrm{~d}) \mathrm{v}$ executa em tempo $O\left(1 / \epsilon^{2}\right)$, visto que Best_Tree gasta um tempo constante. O passo 7(d)vi depende do procedimento Small_Tree, o qual pode ser executado em tempo polinomial em $|I|$. O passo 7 (d)vii é executado 
não mais do que $r+2$ vezes e cada execução gasta tempo polinomial em $|I|$. Logo, o tempo total é limitado por alguma função polinomial em $|I|$ e $2^{O\left(1 / \epsilon^{2}\right)}$.

(b) Análise do erro: Seja $(T, B)$ o par devolvido pelo algoritmo Balance_2CGrafo ${ }_{\epsilon}$. Vamos analisar a aproximação obtida em cada condição de parada.

1. No passo 6 temos que a árvore geradora $T$ encontrada é claramente uma solução ótima.

2. O algoritmo pára no passo 7c. Observe que todos os vértices são pequenos e temos dois casos para analisar.

(a) Se $B=$ Metade então $T$ é uma solução ótima.

(b) Caso contrário, temos $B<$ Metade. Assim, sob as condições do procedimento Add_leaves, existe uma aresta $e$ e duas árvores $T_{1}$ e $T_{2}$ de $T-e$ tais que $w\left(T_{1}\right) \leq B$. Também existe um vértice $v \in T_{2}$ tal que $w\left(T_{1}\right)+w(v)>$ METADE. Isto implica que $B^{*}<w\left(T_{1}\right)+w(v)$. Assim, $\frac{B^{*}-B}{B^{*}} \leq \frac{B^{*}-w\left(T_{1}\right)}{B^{*}}<\frac{w(v)}{B^{*}} \leq \frac{\epsilon \widetilde{B}}{B^{*}} \leq \epsilon$, onde a última desigualdade é válida pelo Lema 3.3.7.

3. No passo 7(d)vii, para analisarmos a aproximação obtida, consideramos duas situações que podem acontecer.

(a) $B^{*}=w(S)$, onde $S \subseteq$ Small:

Considere a árvore geradora $T(r+1)$ construída por Small_Tree no passo $7(\mathrm{~d})$ vi. Se $w\left(C_{1}\right) \leq$ Metade então temos uma solução ótima. Caso contrário, seja $B^{\prime}=$ Balance $(T(r+1))$. A árvore geradora $T(r+1)$ foi construída por Add_leaves e a prova é similar ao Caso $2 \mathrm{~b}$, onde usamos $B^{\prime}$ em vez do balanceamento $B$ devolvido pelo nosso algoritmo. Como $B \geq B^{\prime}$, o resultado segue.

(b) $B^{*}=w\left(v_{1}\right)+\ldots+w\left(v_{k}\right)+\alpha$, onde $k \geq 1, v_{i} \in$ Large para todo $i=1, \ldots, k$ e $\alpha$ é uma soma de pesos de vértices pequenos ( $\alpha$ pode ser $0)$ :

Seja $q_{i}=q_{v_{i}}$ para $1 \leq i \leq k$, e $j^{\prime}=q_{1}+\ldots+q_{k}$. Se $B=$ METADE então temos uma solução ótima $T$. Se $B<$ Metade então devemos ter considerado $T\left(j^{\prime}\right)$ e seu balanceamento $B^{\prime}$ para determinar o par $(T, B)$. 
Seja $B^{\prime}=w\left(v_{j_{1}}\right)+\ldots+w\left(v_{j_{h}}\right)+\beta$ onde $\beta$ é uma soma de pesos de vértices pequenos. Agora considere o momento no passo $7(\mathrm{~d}) \mathrm{v}$ em que $T\left(j^{\prime}\right)$ foi determinada. Devemos, então, analisar dois casos:

i. $\operatorname{Best} 1\left(j^{\prime}\right) \neq$ null: Sob esta condição $T\left(j^{\prime}\right)$ foi construída por Add_leaves. Assim, a análise segue igual ao Caso 2b, usando $B^{\prime} \mathrm{em}$ vez do balanceamento $B$ devolvido pelo algoritmo. Como $B \geq B^{\prime}$ o resultado segue.

ii. $\operatorname{Best1}\left(j^{\prime}\right)=$ null: Agora, para cada conjunto $X \in C\left(j^{\prime}\right)$ o Find_Tree $\left(X, j^{\prime}\right)$ construiu a melhor solução (veja passo 7 (d)iv) sob as condições dos Casos 1 ou 2(a)i deste procedimento. Além disto, $T\left(j^{\prime}\right)$ tem o melhor balanceamento entre todas estas árvores geradoras. Como o conjunto $X=\left\{v_{1}, \ldots, v_{k}\right\}$ foi também considerado, temos $B^{*}=B^{\prime}$ e isto finaliza nossa prova.

Agora discutiremos como podemos usar o algoritmo Balance_2CGrafo $\epsilon_{\epsilon}$ para obter um esquema de aproximação polinomial para o $\mathrm{PCB}_{2}$ restrito a $\Gamma$-grafos onde $w(v)=1$ para todo vértice $v$ que não é um ponto de articulação.

Utilizamos uma variação do algoritmo MaxBal apresentado por Galbiati et al. [GMM97]. Este algoritmo ${ }^{5}$ aqui chamado Balance_Grafo ${ }_{\epsilon}$ reduz gradualmente o número de pontos de articulação no grafo de entrada $G=(V, E)$ até descobrir uma solução ótima ou um grafo 2-conexo, no qual aplicamos nosso algoritmo Balance_2CGrafo ${ }_{\epsilon}$. Observe que os grafos 2-conexos obtidos da nossa classe especial de $\Gamma$-grafos pela redução apresentada no Balance_Grafo ${ }_{\epsilon}$ são sempre $C_{w^{-}}$ grafos. Também note que de qualquer árvore geradora do grafo reduzido é possível reconstruir uma árvore geradora do grafo original cujo balanceamento é maior ou igual.

\footnotetext{
${ }^{5}$ Também utilizamos uma versão do algoritmo MaxBal na Seção 3.1.1 para demonstrar o Teorema 3.1.2.
} 
Algoritmo Balance_Grafo ${ }_{\epsilon}(G, w)$

Entrada: um $\Gamma$-grafo $G$, uma função $w: V \rightarrow \mathbb{Z}_{+}$onde o conjunto dos vértices $v$ com $w(v)>1$ forma um clique.

Saída: uma árvore geradora $T$ de $G$.

1. Se $G$ é um grafo 2-conexo devolva Balance_2CGrafo ${ }_{\epsilon}(G, w)$.

2. Seja $x$ um ponto de articulação de $G$ e sejam $C_{1}, \ldots, C_{h}$ os componentes conexos de $G-x$ obtidos ao remover $x$ de $G$. Seja $w\left(C_{i}\right)$ a soma dos pesos dos vértices de $C_{i}, i=1, \ldots, h$. Sem perda de generalidade, seja $C_{1}$ um componente que maximiza $w\left(C_{i}\right)$.

3. Se $w\left(C_{1}\right) \leq\left\lfloor\frac{W}{2}\right\rfloor$ então devolva qualquer árvore geradora $T$ que conecta $x$ a $C_{1}$ com uma única aresta.

4. Caso contrário, se $w\left(C_{1}\right)>\left\lfloor\frac{W}{2}\right\rfloor$ então

(a) Seja $G^{\prime}$ o subgrafo de $G$ induzido por $x$ e pelos vértices de $C_{1}$; e seja $w^{\prime}$ a nova função peso assim definida: $w^{\prime}(x)=w(x)+\sum_{i=2}^{h} w\left(C_{i}\right)$ e $w^{\prime}(v)=w(v)$ para $v \in V\left(C_{1}\right)$.

/* $G^{\prime}$ tem menos pontos de articulação do que $G * /$

(b) $T^{\prime}=$ Balance_Grafo ${ }_{\epsilon}\left(G^{\prime}, w^{\prime}\right)$.

(c) Transforme $T^{\prime}$ em uma árvore $T$ de $G$ conectando $x$ a uma árvore geradora de cada componente $C_{i}, i \neq 1$.

$/ *$ Balance $(T) \geq \operatorname{Balance}\left(T^{\prime}\right) * /$

(d) Devolva $T$.

\section{fim_do_Algoritmo}

Teorema 3.3.6. O algoritmo Balance_Grafo ${ }_{\epsilon}$ é um esquema de aproximação polinomial para o $\mathrm{PCB}_{2}$ restrito a $\Gamma$-grafos onde $w(v)=1$ para todo vértice $v$ que não é um ponto de articulação.

Prova: Seja $x$ um ponto de articulação de $G$, o grafo de entrada. Sejam $C_{1}, \ldots, C_{h}$ os componentes conexos de $G-x$ obtidos ao remover $x$ de $G$. Seja $w\left(C_{i}\right)$ a soma dos pesos dos vértices de $C_{i}, i=1, \ldots, h$. Sem perda de generalidade, seja $C_{1}$ um componente que maximiza $w\left(C_{i}\right)$. 
Suponha que $w\left(C_{1}\right) \leq\left\lfloor\frac{W}{2}\right\rfloor$. Neste caso, qualquer árvore geradora conectando $x$ a $C_{1}$ por uma única aresta é ótima. Caso contrário, se $w\left(C_{1}\right)>\left\lfloor\frac{W}{2}\right\rfloor$ então usamos

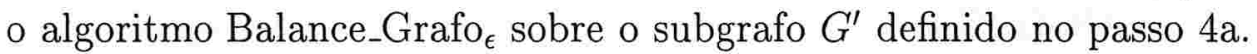

Observe que o novo grafo $G^{\prime}$ tem menos pontos de articulação do que $G$. Assim, se a condição no passo 3 nunca for satisfeita, no final do algoritmo temos um grafo 2conexo. Logo, temos a aproximação exigida pelo Teorema 3.3.5. Para estendermos este resultado para o grafo original $G$, observe que se $T^{\prime}=$ Balance_Grafo $_{\epsilon}\left(G^{\prime}, w^{\prime}\right)$ é uma solução aproximada para $G^{\prime}$, transformada pelo algoritmo em uma árvore geradora $T$ para $G$, então

$$
\operatorname{Balance}\left(T^{\prime}\right)=\max _{e \in E\left(T^{\prime}\right)} f\left(e, T^{\prime}\right) \leq \max _{e \in E(T)} f(e, T)=\operatorname{Balance}(T),
$$

pois qualquer aresta de $T^{\prime}$ permanece como uma aresta de $T$.

De acordo com as observações efetuadas, concluímos que se $T^{\prime}$ é uma solução para $G^{\prime}$ com erro relativo no máximo $\epsilon$, onde $0<\epsilon<1$, então $T$ é uma solução para $G$ com a mesma aproximação.

Uma conseqüência imediata do Teorema 3.3.6 é o seguinte resultado.

Corolário 3.3.4. O algoritmo Balance_Grafo ${ }_{\epsilon}$ é um esquema de aproximação polinomial para o $\mathrm{PCB}_{2}$ restrito a $C_{w}$-grafos.

Comparamos a seguir as propriedades de aproximação das versões com e sem pesos do $\mathrm{PCB}_{2}$. No que segue, ainda utilizamos este problema na sua versão equivalente como problema de árvore geradora.

\subsubsection{Removendo Pesos}

Crescenzi et al. [CST01] estudaram a complexidade relativa às propriedades de aproximação de três possíveis versões de um problema de otimização: aquela com pesos arbitrários, com pesos polinomialmente limitados e sem pesos. Comentamos este fato na Seção 2.5. Estes resultados, com pequenas modificações, podem ser estendidos para incluir o $\mathrm{PCB}_{2}$ e outros problemas de maximização que pertencem a uma classe um pouco diferente da classe denominada problemas-subconjuntos, a qual é descrita a seguir. 
Definição 3.3.6. Um problema de otimização $\mathcal{P}$ é um problema-subconjunto estendido se:

- Qualquer instância $x$ de $\mathcal{P}$ é uma tripla $\left(U, x^{\prime}, w\right)$ onde $U=\left\{u_{1}, \ldots, u_{n}\right\}$ é um conjunto de objetos, $x^{\prime}$ é uma cadeia (possivelmente vazia), e $w: U \rightarrow \mathbb{N}$ atribui um peso $w(u)$ para qualquer objeto $u \in U$.

- A qualquer solução y $\in \operatorname{sol}_{\mathcal{P}}(x)$ é possível associar para cada $a \in y$ um subconjunto $S_{a}^{\prime} \subseteq U$, tal que a medida de y é igual a $\max _{a \in y} \sum_{u \in S_{a}^{\prime}} w(u)$.

Por exemplo, considere o $\mathrm{PCB}_{2}$. Uma instância desse problema é uma tripla $(G, \lambda, w)$ onde $G=(V, E)$ é um grafo, $w: V \rightarrow \mathbb{N}$ é uma função que atribui pesos aos vértices de $G$, e $\lambda$ é uma cadeia vazia. Uma solução é uma árvore geradora $T$ de $G$ e para cada aresta $e=(x, y)$ em $T$ associamos o subconjunto $V\left(T_{x}\right) \subseteq V$. Lembre-se que a remoção de $e=(x, y)$ em $T$ dá origem a duas subárvores $T_{x} \mathrm{e}$ $T_{y}$, onde $T_{x}$ é a subárvore cuja soma dos pesos nos vértices $S_{x}$ é no máximo $\left\lfloor\frac{W}{2}\right\rfloor$ e $W=w(V)$, como convencionamos. Mais ainda, a medida é $\max _{e \in T} \sum_{u \in T_{x}} w(u)$.

Aplicamos a mesma noção de problema refinado (veja Definição 2.5.2) para a classe de problemas subconjunto estendidos. Assim, dizemos que um problema subconjunto estendido é refinado se, para qualquer instância $\left(U, x^{\prime}, w\right)$, se $y$ é uma solução viável, então, para qualquer função $w^{\prime}: U \rightarrow \mathbb{N}$, y é também uma solução viável para $\left(U, x^{\prime}, w^{\prime}\right)$.

Definição 3.3.7. Seja $\mathcal{P}$ um problema subconjunto estendido e $p$ um polinômio. Denotamos por $\mathcal{P}^{p}$ a restrição de $\mathcal{P}$ para instâncias $x=\left(U, x^{\prime}, w\right)$ tais que a soma de pesos $\sum_{u \in U} w(u)$ é no máximo $p(|x|)$.

Agora podemos concluir o seguinte resultado.

Teorema 3.3.7. Seja $\mathcal{P}$ um problema-subconjunto estendido de maximização em $A P X$. Existe um polinômio $p$ tal que se $\mathcal{P}^{p}$ tem uma $r$-aproximação, para algum $r>1$, então $\mathcal{P}$ tem uma $(r+1 / n)$-aproximação, onde $n$ é o tamanho da instância.

Prova: Seja $\mathcal{P}$ um problema de maximização. Seja $A$ um algoritmo $r_{0^{-}}$ aproximado para $\mathcal{P}$ e $v(x)=m(x, A(x))$. Assim, para qualquer instância $x$, temos que

$$
v(x) \leq o p t(x) \leq r_{0} v(x) .
$$


Seja $x=\left(U, x^{\prime}, w\right)$ uma instância de tamanho $n$. Note que $|U| \leq n$. Definimos, então uma nova função peso $\tilde{w}$, tal que, para qualquer $u \in U$,

$$
\tilde{w}(u)=\left\lfloor\frac{w(u) n^{2}}{v(x)}\right\rfloor
$$

Observe que, como $\mathcal{P}$ é refinado, o conjunto de soluções viáveis para $\left(U, x^{\prime}, w\right)$ é igual aquele de $\left(U, x^{\prime}, \tilde{w}\right)$. Além disto, podemos assumir sem perda de generalidade que $w(u) \leq r_{0} v(x)$ para qualquer $u \in U$. Desta forma, temos que $\tilde{w}(u) \leq r_{0} n^{2}$ para qualquer $u \in U$. Assim, $\tilde{x}=\left(U, x^{\prime}, \tilde{w}\right)$ é uma instância de $\mathcal{P}^{p}$, onde $p=r_{0} n^{3}$.

Da definição de $\tilde{w}$ temos que, para qualquer solução viável $y$,

$$
m(x, y) \geq m(\tilde{x}, y) v(x) / n^{2} .
$$

Além disto, seja $a \in y$ tal que $m(x, y)=\sum_{u \in S_{a}^{\prime}} w(u)$. Então,

$$
\begin{aligned}
m(x, y) & =\Sigma_{u \in S_{a}^{\prime}} w(u) \leq \Sigma_{u \in S_{a}^{\prime}} \frac{v(x)}{n^{2}}\left\lceil\frac{w(u) n^{2}}{v(x)}\right\rceil \leq \frac{v(x)}{n^{2}} \Sigma_{u \in S_{a}^{\prime}}\left(\left\lfloor\frac{w(u) n^{2}}{v(x)}\right\rfloor+1\right) \\
& =\left(v(x) / n^{2}\right)\left(m(\tilde{x}, y)+\left|S_{a}^{\prime}\right|\right) \leq\left(v(x) / n^{2}\right)(m(\tilde{x}, y)+n) .
\end{aligned}
$$

Por outro lado, podemos assumir que

$$
m(x, y) \geq v(x)
$$

caso contrário poderíamos substituir $y$ pela solução computada pelo algoritmo de aproximação $A$. Logo,

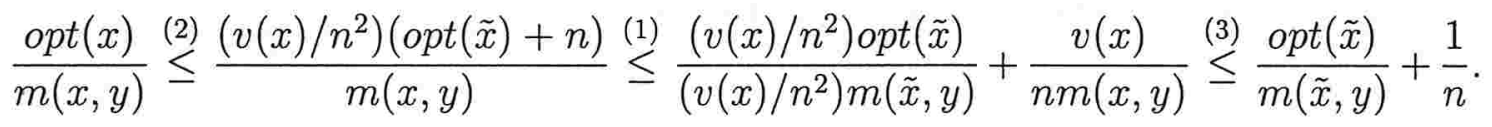

Assim, se $y$ é uma solução $r$-aproximada para $\tilde{x}$, então $y$ é uma solução $\left(r+\frac{1}{n}\right)$-aproximada para $x$. Isto conclui nossa prova.

Em conseqüência, podemos também concluir o seguinte resultado para problemas-subconjunto estendidos refinados. 
Corolário 3.3.5. Seja $\mathcal{P}$ um problema-subconjunto estendido refinado de maximização tal que $\mathcal{P} \in A P X$. Então existe um polinômio $p$ tal que $\mathcal{P}$ e $\mathcal{P}^{p}$ têmo mesmo limiar de aproximação.

Naturalmente, podemos aplicar o Corolário 3.3.5 para o $\mathrm{PCB}_{2}$. Contudo, podemos ter um resultado ainda mais forte como demonstramos a seguir. Nesses dois resultados denotamos por $1 \mathrm{PCB}_{2}$ a versão do $\mathrm{PCB}_{2}$ sem pesos.

Teorema 3.3.8. Para qualquer polinômio $p, \mathrm{PCB}_{2}^{p} \leq_{A P}^{1} 1 \mathrm{PCB}_{2}$.

Prova: Seja $G=(V, E)$ um grafo e $w: V \rightarrow N$ uma função peso polinomialmente limitada. Podemos, então, definir um novo grafo $G^{\prime}=\left(V^{\prime}, E^{\prime}\right)$ como segue. Para qualquer vértice $u_{t} \in V$ tal que $w\left(u_{t}\right)=k, V^{\prime}$ tem $k$ vértices $u_{t}^{1}, \ldots, u_{t}^{k}$, $\operatorname{com}\left(u_{t}^{i}, u_{t}^{1}\right) \in E^{\prime}, i=2, \ldots, k$. Além disto, se $\left(u_{t}, u_{t^{\prime}}\right) \in E$ então $\left(u_{t}^{1}, u_{t^{\prime}}^{1}\right) \in E^{\prime}$. Observe que dado $G$ e $w$, o grafo $G^{\prime}$ pode ser construído em tempo polinomial. É fácil perceber que se $T^{\prime}$ é uma solução de $G^{\prime}$ com balanceamento $b^{\prime}$, então podemos computar em tempo polinomial uma solução $T$ de $G$ cujo balanceamento é pelo menos $b^{\prime}$. É também óbvio que se $T$ é uma solução de $(G, w)$ com balanceamento $b$, então podemos conseguir uma solução para $G^{\prime}$ com mesmo balanceamento.

Corolário 3.3.6. $O \mathrm{PCB}_{2}$ e o $1 \mathrm{PCB}_{2}$ têm o mesmo limiar de aproximação.

Prova: Pelo Corolário 3.3.5, temos que existe um polinômio $p$ tal que $\mathrm{PCB}_{2}$ e $\mathrm{PCB}_{2}^{p}$ têm o mesmo limiar de aproximação (veja Definição 2.3.5). Usando o Teorema 3.3.8 temos o resultado desejado.

\subsection{Conclusão}

Neste capítulo, estudamos o problema Max 2-Partição Conexa Balanceada. Vimos que do ponto de vista algorítmico, é suficiente resolver este problema para grafos 2-conexos, já que algoritmos para tais grafos podem ser usados para resolver este problema quando o grafo de entrada é conexo. Em consequêcia, exibimos alguns resultados sobre o $\mathrm{PCB}_{2}$ restrito a grafos 2-conexos. Por exemplo, mostramos que, sob a hipótese de que $\mathrm{P} \neq \mathrm{NP}$, a versão de decisão do $\mathrm{PCB}_{2}$ restrito a 
grafos 2-conexos é um problema fortemente NP-completo, e portanto não admite um FPTAS.

Neste contexto da inaproximabilidade do $\mathrm{PCB}_{2}$ também provamos que sobre grafos conexos com $n$ vértices, este problema não admite um algoritmo $(1+\epsilon)$ aproximado onde $\epsilon \leq \frac{1}{n^{2}}$, a menos que $\mathrm{P}=\mathrm{NP}$ (mesmo para grafos bipartidos).

Uma releitura do algoritmo (4/3)-aproximado desenvolvido por Chlebíková [Chl96] também foi exibida e com nossa análise parametrizada descrevemos classes de grafos para as quais as soluções encontradas se aproximam assintoticamente do ótimo. Adicionalmente, mostramos que a razão de aproximação deste algoritmo é justa mesmo para grafos 3-conexos. Além disto, apresentamos para grafos 3-conexos um algoritmo baseado em contrações de arestas que pode devolver soluções com qualidade melhor do que a razão 4/3 garantida pelo algoritmo anterior.

Uma questão importante relacionada ao $\mathrm{PCB}_{2}$ é a existência de um esquema de aproximação polinomial. Nesta direção, exibimos um $\mathrm{PTAS}$ para o $\mathrm{PCB}_{2}$ restrito a duas classes de grafos que chamamos de $\Gamma$-grafos e $C_{w}$-grafos. Nossa motivação para estudar estes grafos advém de algumas topologias de redes de longa distância (wide area networks) que são casos especiais desta classe de grafos. O esquema de aproximação que apresentamos é baseado nas idéias usadas no FPTAS para o problema Max 0/1 Mochila, desenvolvido por Ibarra, Kim [IK75] e Lawler [Law79], combinadas com idéias de um algoritmo $\frac{9}{8}$-aproximado exibido por Galbiati et al. [GMM97]. Até onde sabemos, nosso algoritmo é o primeiro a aplicar estas idéias para os problemas de partição estudados nesta tese.

Outro resultado que obtivemos foi que as versões com e sem pesos do $\mathrm{PCB}_{2}$ (para grafos conexos) possuem o mesmo limiar de aproximação.

Uma generalização natural do $\mathrm{PCB}_{2}$ é o problema da $q$-Partição Conexa Balanceada, para $q \geq 3$. Este é o assunto do próximo capítulo. 
Capítulo 4

\title{
Sobre $q$-Partições Conexas Balanceadas
}

\author{
"Se você quer realmente entender alguma coisa \\ tente modificá-la." \\ (Kent Lewin)
}

Um caminho natural para se generalizar o problema da 2-partição conexa balanceada é considerar o conceito de $q$-partição conexa balanceada, onde $q \geq 2$. Tal como no caso da partição em duas classes, basta impor que cada uma das $q$ classes induza um subgrafo conexo do grafo de entrada, e que a partição seja a mais balanceada possível. Mais formalmente, a generalização a que estamos nos referindo e que será o tema deste capítulo é a seguinte.

\section{Max $q$-Partição Conexa Balanceada $\left(\mathrm{PCB}_{q}\right)$}

Instância: um grafo $G=(V, E)$ e uma função $w: V \rightarrow \mathbb{Z}_{+}$.

Solução viável: uma $q$-partição conexa $\left(V_{1}, V_{2}, \ldots, V_{q}\right)$ de $G$.

Função: $\min \left\{w\left(V_{1}\right), w\left(V_{2}\right), \ldots, w\left(V_{q}\right)\right\}$.

Objetivo: maximizar.

Referenciamos o caso especial onde todos os vértices têm peso unitário como $\mathrm{PCB}_{q}$ sem pesos.

Esta generalização do particionamento conexo balanceado tem aplicação nas 
áreas de processamento de imagem [AS73, LPS89, LPS93, MRS95], paginação em bancos de dados [BP95], análise de 'clusters' [MSN97], etc.

Um exemplo interessante de aplicação descrito por Becker et al. [BLLS01] é o problema do Patrulhamento Fluvial. Neste problema, as duas margens de um rio que corta uma cidade são conectadas por barcos em várias estações, distribuídas entre as duas margens; a questão é decidir como a polícia da cidade deve atribuir um conjunto de estações de barcos para $q$ patrulhas de modo que cada estação seja vigiada por exatamente uma patrulha. Sabe-se que cada patrulha está apta a se mover dentro de sua área ou por meio de bicicleta ao longo de uma margem ou de barco. Assim, cada área a ser atribuída deve "ser conexa"; e além disso, o patrulhamento, quando medido pela freqüência dos crimes observados dentro das áreas vigiadas, deve ser tão 'balanceado' quanto possível.

Outra aplicação [BLLS98] que podemos citar para motivar o estudo do $\mathrm{PCB}_{q}$ é a definição das áreas de responsabilidade de supervisores em uma fábrica. Neste caso, temos unidades individuais (vértices) cujo peso significa o tempo exigido para inspeção e desejamos atribuir porções (componentes) da fábrica para $q$ pessoas distintas de modo que cada parte seja conexa (para garantir concentração espacial da atividade do supervisor). Também busca-se uma atribuição tal que as cargas de trabalho dos supervisores sejam aproximadamente iguais (balanceadas).

Antes de discutirmos estratégias de resolução para o $\mathrm{PCB}_{q}$, vamos investigar a sua complexidade computacional. Apresentamos na próxima seção alguns resultados dessa natureza.

\subsection{Complexidade Computacional}

No caso do $\mathrm{PCB}_{q}$ sem pesos, ressaltamos inicialmente o seguinte resultado, devido a Lovász [Lov77] (veja também Györi [Gyo78]).

Teorema 4.1.1. Seja $G$ um grafo $k$-conexo com $n$ vértices, $k \geq 2$, e sejam $n_{1}, n_{2}, \ldots, n_{k}$ naturais positivos tais que $n_{1}+n_{2}+\ldots+n_{k}=n$. Então $G$ tem uma $k$-partição conexa $\left(V_{1}, V_{2}, \ldots, V_{k}\right)$ tal que $\left|V_{i}\right|=n_{i}$ para $1=1,2, \ldots, k$.

A prova apresentada por Lovász usa conceitos de topologia, enquanto a prova apresentada por Györi [Gyo78] utiliza apenas conceitos da teoria de grafos. Na verdade, um resultado mais forte que o acima foi apresentado por esses autores: 
além da exigência sobre as cardinalidades, pode-se escolher a priori $k$ vértices distintos, digamos $v_{1}, v_{2}, \ldots, v_{k}$, e exigir que a partição $\left(V_{1}, V_{2}, \ldots, V_{q}\right)$ seja tal que $v_{i} \in V_{i}$ para $1=1,2, \ldots, k$. Garante-se que uma tal partição existe se $G$ é $k$-conexo.

Ressaltamos que estas provas não fornecem imediatamente um algoritmo polinomial para encontrar essa $k$-partição mencionada. Algoritmos eficientes surgiram apenas nos anos 90. Inicialmente, para os casos $k=2$ e $k=3$ [STN90a, STN ${ }^{+} 90 \mathrm{~b}$ ]. Depois, Jun Ma e Shao Ma [MM94] exibiram um algoritmo polinomial de complexidade $O\left(k^{2} n^{2}\right)$ para grafos $k$-conexos, $k \geq 2$. Em 1997, Nakano, Rahman e Nishizeki [NRN97] obtiveram um algoritmo linear para encontrar uma 4-partição conexa em grafos planares 4-conexos.

Uma pergunta natural neste contexto é se existe uma $(k+1)$-partição conexa para grafos $k$-conexos, nas mesmas condições especificadas no Teorema 4.1.1. Por tais condições, entendamos as restrições de cardinalidade de cada conjunto da partição. Recentemente, Diwan [Diw03] mostrou que o grafo bipartido completo $K_{k, k+2}$ é um exemplo de um grafo $k$-conexo que não possui uma $(k+1)$-partição conexa. Neste artigo são considerados grafos $k$-conexos $G$ com grau máximo $\Delta(G) \leq(k+1)$ : é mostrado que tais grafos não têm necessariamente uma $(2 k+1)$ partição conexa, e é provado que grafos 2-conexos com $\Delta(G) \leq 3$ possuem uma 4-partição conexa. Assim, esse resultado para grafos 2-conexos é o melhor possí vel.

Com relação ao $\mathrm{PCB}_{q}$, na sua versão geral (com pesos), não encontramos muitos resultados na literatura. Dentre aqueles que encontramos, mencionamos a existência de algoritmos polinomiais para o $\mathrm{PCB}_{q}$ restrito a árvores [PS81] e escadas (ladders) [BLLS01].

A intratabilidade do $\mathrm{PCB}_{q}$ para $q \geq 2$ segue imediatamente para todos os casos descritos nas Seções 3.1 e 3.1.1 (já que o caso $q=2$ é um caso especial que já é difícil). Ressaltamos porém que há um resultado ainda mais forte, devido a Dyer e Frieze [DF85]: estes autores demonstraram a NP-completude do $\mathrm{PCB}_{q}$ sem pesos para qualquer $q \geq 2$ fixo (mesmo para grafos bipartidos).

Becker et al. [BLLS98] desenvolveram dois algoritmos de aproximação para o $\mathrm{PCB}_{q}$ restrito às grades $G_{m \times n}, \operatorname{com} n \geq 3$. Neste caso, a medida adotada foi o erro relativo (veja Definição 2.4.2). No primeiro algoritmo, limites para o erro relativo são obtidos apenas sob algumas condições que são verificadas a 'posteriori' (isto é após a execução do algoritmo). No segundo, a estimativa do erro pode ser 
obtida se certas hipóteses verificáveis a 'priori' são satisfeitas. No entanto, apenas quando essas hipóteses são relaxadas, é possível determinar limites sobre o erro, não fornecendo resultados de impacto. Apenas no caso $q=2$ pode-se garantir uma (3/2)-aproximação, considerando-se a razão como medida de erro. Além disto, utiliza fortes condições para deduzir que tal algoritmo é assintoticamente exato.

Antes de investigarmos a aproximabilidade do $\mathrm{PCB}_{q}$, provamos um resultado de grande utilidade neste capítulo.

Teorema 4.1.2. Seja $I=(G, w)$ uma instância do $\mathrm{PCB}_{q}, q \geq 2$, que consiste de um grafo 2-conexo $G=(V, E)$ e uma função $w: V \rightarrow \mathbb{Z}_{+}$tal que $\max \{w(v): v \in$ $V\} \geq(1 / q) w(V)$. Seja $v^{*}=\arg \max \{w(v): v \in V\}$. Então I tem uma solução ótima $\left(V_{1}^{*}, \ldots, V_{q}^{*}\right)$ tal que $V_{i}^{*}=\left\{v^{*}\right\}$ para algum $i, 1 \leq i \leq q$.

Prova: Seja $P=\left(V_{1}, V_{2}, \ldots, V_{q}\right)$ uma solução ótima para $I$ tal que o conjunto da partição que contém $v^{*}$, digamos $V_{j}$, tem a menor cardinalidade possível. Suponha que $\left|V_{j}\right|>1$. Como $G$ é 2-conexo, então existe um vértice $v^{\prime} \in V_{j}, v^{\prime} \neq v^{*}$, adjacente a algum vértice $v^{\prime \prime} \notin V_{j}$. Suponha que $v^{\prime \prime} \in V_{t}$.

Seja $T$ uma árvore geradora de $G\left[V_{j}\right]$. Agora considere o caminho em $T$ que vai de $v^{\prime}$ até $v^{*}$ e chame de $f$ a primeira aresta desse caminho. Seja $T^{\prime}$ a árvore de $T-f$ que contém $v^{\prime}$. Sejam $V_{j}^{\prime}=V_{j} \backslash V\left(T^{\prime}\right)$ e $V_{t}^{\prime}=V_{t} \cup V\left(T^{\prime}\right)$. Note que $G\left[V_{j}^{\prime}\right]$ e $G\left[V_{t}^{\prime}\right]$ são ambos conexos (a aresta $v^{\prime} v^{\prime \prime}$ pode ser usada para conectar a árvore $T^{\prime}$ ao subgrafo $\left.G\left[V_{t}\right]\right)$. Chame de $P^{\prime}$ a nova $q$-partição conexa obtida de $P$ substituindo $V_{j}$ por $V_{j}^{\prime}$ e $V_{t}$ por $V_{t}^{\prime}$. Vamos mostrar que $P^{\prime}$ contradiz a escolha de $P$.

Suponha sem perda de generalidade que $w\left(V_{1}\right)=\min \left\{w\left(V_{i}\right) \mid 1 \leq i \leq q\right\}$. Claramente, $V_{1} \neq V_{j}$. Inicialmente, note que $v^{*} \in V_{j}^{\prime}$, e portanto, $w\left(V_{j}^{\prime}\right) \geq\left(\frac{1}{q}\right) w(V)$. Agora vamos considerar $V_{t}^{\prime}$ e analisar dois casos:

(a) $t \neq 1$. Neste caso, a medida da nova partição $P^{\prime}$ é igual à medida de $P$.

(b) $t=1$. Como $w\left(V_{1}^{\prime}\right)>w\left(V_{1}\right)$ e $w\left(V_{j}^{\prime}\right) \geq(1 / q) w(V)$, e a medida da nova partição $P^{\prime}$ não pode ser maior do que a medida de $P$, então deve existir um outro conjunto de $P$ com peso $w\left(V_{1}\right)$, e neste caso, $P^{\prime}$ tem a mesma medida que $P$. 
Em ambos os casos concluímos que $P^{\prime}$ é uma solução ótima. Mas $P^{\prime}$ contradiz a escolha de $P$, visto que $\left|V_{j}^{\prime}\right|<\left|V_{j}\right|$. Logo, devemos ter $\left|V_{j}\right|=1$, como queríamos demonstrar.

Apresentamos nas próximas seções estudos sobre a aproximabilidade do $\mathrm{PCB}_{q}$, $q \geq 3$, para grafos mais gerais do que grades $G_{m \times n}$ e árvores (classes já estudadas). Começamos investigando o $\mathrm{PCB}_{3}$.

\subsection{Max 3-Partição Conexa Balanceada}

Nesta seção, abordamos as propriedades de aproximação do $\mathrm{PCB}_{3}$ restrito a grafos $k$-conexos para $k=2$ e $k=3$. Como passo inicial, analisamos a complexidade computacional desses casos especiais.

Para este propósito, usamos o fato de que a versão de decisão do $\mathrm{PCB}_{2}$ restrito a grafos 2-conexos é um problema fortemente NP-completo, como provamos no Teorema 3.1.1. Denotamos por $\mathrm{DPCB}_{2}$ esse problema. Analogamente, denotamos por $\mathrm{DPCB}_{3}$ a versão de decisão do $\mathrm{PCB}_{3}$ restrito a grafos 3-conexos.

Teorema 4.2.1. A versão de decisão do Max 3-Partição Conexa Balanceada restrito a grafos 3-conexos é um problema NP-completo.

Prova: Seja $I=(G, w, m)$ uma instância do $\mathrm{DPCB}_{2}$ que consiste de um grafo 2-conexo $G=(V, E)$, uma função $w: V \rightarrow \mathbb{Z}_{+}$e um inteiro positivo $m$. O objetivo é decidir se esta instância tem uma solução com medida pelo menos $m$.

Construímos uma instância $I^{\prime}=\left(G^{\prime}, w^{\prime}, m\right)$ do $\mathrm{DPCB}_{3}$ que consiste de um grafo 3-conexo $G^{\prime}=\left(V^{\prime}, E^{\prime}\right)$, com $V^{\prime}=V \cup\left\{v^{\prime}\right\}$, onde $v^{\prime} \notin V$, e $E^{\prime}=E \cup\left\{v^{\prime} u: u \in V\right\}$, e uma função $w^{\prime}$ sobre os vértices de $G^{\prime}$ tal que: $w^{\prime}\left(v^{\prime}\right)=w(V) / 2$ e $w^{\prime}(v)=w(v)$ para todo $v$ em $V$. É óbvio que $G^{\prime}$ pode ser construído em tempo polinomial no tamanho de $I$, e que $G^{\prime}$ é 3-conexo.

Vamos mostrar que a instância $I$ do $\mathrm{DPCB}_{2}$ possui uma 2-partição conexa com medida pelo menos $m$ se só se a instância $I^{\prime}$ do $\mathrm{DPCB}_{3}$ tem uma 3-partição conexa com medida pelo menos $m$.

Seja $P=(X, Y)$ uma 2-partição conexa de $G$ com medida pelo menos $m$. Neste caso, $\left(X, Y,\left\{v^{\prime}\right\}\right)$ é uma 3-partição conexa de $G^{\prime}$ com medida pelo menos $m$. 
Agora, suponha que $P^{\prime}=\left(X^{\prime}, Y^{\prime}, Z^{\prime}\right)$ seja uma 3-partição conexa de $G^{\prime}$ com medida $m^{\prime}$, onde $m^{\prime} \geq m$. Sem perda de generalidade, suponha que $Z^{\prime}$ contenha $v^{\prime}$. Suponha também que $w^{\prime}\left(X^{\prime}\right) \leq w^{\prime}\left(Y^{\prime}\right)$. Como $w^{\prime}\left(Z^{\prime}\right) \geq(1 / 3) w^{\prime}\left(V^{\prime}\right)$, temos que $m^{\prime}=w^{\prime}\left(X^{\prime}\right)$. Seja $R=Z^{\prime} \backslash\left\{v^{\prime}\right\}$. Se $R=\emptyset$, então $\left(X^{\prime}, Y^{\prime}\right)$ é uma 2-partição conexa de $G$ com medida $m^{\prime}$. Se $R \neq \emptyset$, como $G$ é 2-conexo, então podemos distribuir os vértices de $R$ entre os conjuntos $X^{\prime}$ e $Y^{\prime}$, de modo que os novos conjuntos $X^{\prime} \cup R_{1}$ e $Y^{\prime} \cup R_{2}$, onde $R_{1} \cup R_{2}=R$, induzam subgrafos conexos de $G$. Neste caso, $\left(X^{\prime} \cup R_{1}, Y^{\prime} \cup R_{2}\right)$ é uma 2-partição conexa de $G$ com medida pelo menos $m^{\prime}$. Como $m^{\prime} \geq m$, o resultado segue.

Do teorema acima deduzimos imediatamente o seguinte resultado.

Corolário 4.2.1. A versão de decisão do $\mathrm{PCB}_{3}$ restrito a grafos 2-conexos é um problema NP-completo.

Na próxima seção apresentamos algoritmos de aproximação para o $\mathrm{PCB}_{3}$ restrito a grafos 2-conexos e 3-conexos.

\subsubsection{Grafos 2-conexos e 3-conexos}

Nesta seção apresentamos dois algoritmos para encontrar 3-partições conexas: o BalBicon 3 , para grafos 2-conexos; e o BalCon 3 para grafos 3-conexos.

$\mathrm{O}$ algoritmo BalBicon $_{3}$ baseia-se nas idéias usadas no algoritmo BalBicon ${ }_{2}$ apresentado na Seção 3.3. Já o algoritmo $\mathrm{BalCon}_{3}$ baseia-se nas idéias usadas no algoritmo Part3Con apresentado nessa mesma seção.

Antes de apresentarmos esses algoritmos provamos um resultado que será útil para mostrarmos a qualidade de aproximação dos algoritmos que veremos nesta e na Seção 4.3.

Teorema 4.2.2. Seja $I=(G, w)$ uma instância do $\mathrm{PCB}_{q}, q \geq 3$, que consiste de um grafo 2-conexo $G=(V, E)$ e uma função $w: V \rightarrow \mathbb{Z}_{+}$tal que $\max \{w(v) \mid v \in$ $V\} \geq \frac{1}{q} w(V)$. Se o problema $P C B_{q-1}$ pode ser $r$-aproximado, para algum $r$, então existe uma soluçâo r-aproximada para a instância I do $\mathrm{PCB}_{q}$.

Prova: Seja $x=\arg \max \{w(v) \mid v \in V\}$ e seja $I^{\prime}=\left(G^{\prime}, w^{\prime}\right)$ uma instância do $\mathrm{PCB}_{q-1}$, onde $G^{\prime}=G-x$ e $w^{\prime}$ é a restrição de $w$ aos vértices de $G^{\prime}$. 
Pelo Teorema 4.1 .2 sabemos que existe uma solução ótima $\left(V_{1}^{*}, \ldots, V_{q}^{*}\right)$ para a instância $I$ tal que $V_{i}^{*}=\{x\}$ para algum $1 \leq i \leq q$. Sem perda de generalidade, podemos supor que $V_{1}^{*}=\{x\}$. Neste caso, $\left(V_{2}^{*}, \ldots, V_{q}^{*}\right)$ é uma solução ótima para a instância $I^{\prime}$ do $\mathrm{PCB}_{q-1}$. Como $w(x) \geq 1 / q$, temos que opt $(I)=\operatorname{opt}\left(I^{\prime}\right)$.

Seja $\left(V_{1}^{\prime}, \ldots, V_{q-1}^{\prime}\right)$ uma solução $r$-aproximada para a instância $I^{\prime}$ do $\mathrm{PCB}_{q-1} \mathrm{e}$ seja $m^{\prime}$ a sua medida. Então

$$
\frac{\operatorname{opt}\left(I^{\prime}\right)}{m^{\prime}} \leq r
$$

Observe que $\left(\{x\}, V_{1}^{\prime}, \ldots, V_{q-1}^{\prime}\right)$ é uma solução para a instância $I$ do $\operatorname{PCB}_{q}$. Então se $m$ é a sua medida, temos que $m=m^{\prime}$, já que $w(x) \geq 1 / q$. Substituindo opt $\left(I^{\prime}\right)$ por $\operatorname{opt}(I)$ e $m^{\prime}$ por $m$ na desigualdade acima, temos que

$$
\frac{\operatorname{opt}(I)}{m} \leq r
$$

como queríamos mostrar.

A seguir descrevemos o algoritmo BalBicon $_{3}$. Este separa um vértice de peso máximo do grafo de entrada, e aplica o algoritmo BalBicon 2 ou BalCon $_{2}$ no grafo que resulta após a remoção desse vértice.

Algoritmo $\operatorname{BalBicon}_{3}(G, w)$

Entrada: um grafo 2-conexo $G=(V, E)$ e uma função $w: V \rightarrow \mathbb{Z}_{+}$.

Saída: uma 3-partição conexa $(X, Y, \bar{Y})$ de $G$.

1. Seja $v_{1}=\arg \max \{w(v) \mid v \in V\}$.

2. $X=\left\{v_{1}\right\}$.

3. Seja $G^{\prime}=G-X$ e $w^{\prime}$ a restrição de $w$ aos vértices de $G^{\prime}$.

4. Se $G^{\prime}$ é 2-conexo então $(Y, \bar{Y})=\operatorname{BalBicon}_{2}\left(G^{\prime}, w^{\prime}\right)$;

5. senão $(Y, \bar{Y})=\operatorname{BalCon}_{2}\left(G^{\prime}, w^{\prime}\right)$.

6. Devolva $(X, Y, \bar{Y})$.

fim_do_Algoritmo 
Analisamos a seguir o desempenho do algoritmo BalBicon ${ }_{3}$ quando aplicado a um grafo 3-conexo.

Teorema 4.2.3. Seja $I=(G, w)$ uma instância do $\mathrm{PCB}_{3}$ que consiste de um grafo 3-conexo $G=(V, E)$ e uma função $w: V \rightarrow \mathbb{Z}_{+}$. Seja $p_{\max }=\max \{w(v) \mid v \in V\}$. Então o algoritmo $\mathrm{BalBicon}_{3}$ aplicado à instância I devolve uma 3-partição conexa de $G$ com medida $m$ tal que:

(a) Se $p_{\max } \geq \frac{1}{3} w(V)$ então $\frac{\operatorname{opt}(I)}{m} \leq \frac{4}{3}$.

(b) $S e \frac{2}{9} w(V) \leq p_{\max }<\frac{1}{3} w(V)$ então $\frac{\operatorname{opt}(I)}{m} \leq \frac{3}{2}$.

(c) $S e \frac{1}{s} w(V) \leq p_{\max }<\frac{2}{9} w(V)$ então $\frac{\operatorname{opt}(I)}{m} \leq \frac{s}{3}$.

Ademais, o algoritmo $\mathrm{BalBicon}_{3}$ pode ser implementado de modo que ele fique com complexidade polinomial.

Prova: A complexidade computacional do $\mathrm{BalBicon}_{3}$ está diretamente relaci-

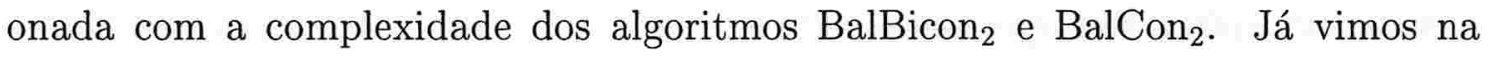
Seção 3.3 que estes podem ser implementados de modo que fiquem com complexidade polinomial. Também é imediato que este algoritmo constrói uma 3-partição conexa de $G$.

Analisamos a seguir a qualidade da solução $(X, Y, \bar{Y})$ devolvida. Primeiramente observamos que pelo passo 2 do algoritmo temos que $X=\left\{v_{1}\right\}$, onde $v_{1}=\arg \max \{w(v) \mid v \in V\}$. Consideramos agora dois casos.

(a) $p_{\max } \geq \frac{1}{3} w(V)$.

Neste caso, pelo Teorema 4.2.2 e pelo Corolário 3.3.1, concluímos que

$$
\frac{\operatorname{opt}(I)}{m} \leq \frac{4}{3}
$$

(b) $\frac{1}{s} w(V) \leq p_{\max }<\frac{1}{3} w(V)$.

Então $\frac{1}{s} w(V) \leq w(X)<\frac{1}{3} w(V)$. Sejam $G^{\prime}$ e $w^{\prime}$ os objetos definidos no passo 3. Como $G^{\prime}$ é 2-conexo, pelo passo 4 temos que $(Y, \bar{Y})=\operatorname{BalBicon}_{2}\left(G^{\prime}, w^{\prime}\right)$. Neste caso, $w\left(V^{\prime}\right)=w(V)-w(X)>(2 / 3) w(V)$. Como $w(v)<(1 / 3) w(V)$ 
para todo $v$ em $V^{\prime}$, então $w(v)<(1 / 2) w\left(V^{\prime}\right)$. Logo, usando o Teorema 3.3.2, temos que $\min \{w(Y), w(\bar{Y})\} \geq(1 / 3) w\left(V^{\prime}\right)>(2 / 9) w(V)$. Então, $m \geq \min \left\{\frac{1}{s}, \frac{2}{9}\right\} w(V)$, e portanto,

$$
\frac{\operatorname{opt}(I)}{m} \leq \frac{3}{2} \text {, se } s \leq 9 / 2 ; \text { e } \frac{\operatorname{opt}(I)}{m} \leq \frac{s}{3} \text {, se } s \geq 9 / 2 .
$$

Com pequenas modificações na prova acima, e observando que quando o grafo $G^{\prime}$ (construído no passo 3) não é 2-conexo, possivelmente a solução devolvida pode ter medida igual a do vértice de peso mínimo, segue imediatamente o seguinte resultado. Observamos, no entanto, que mesmo neste caso, para muitas instâncias a solução pode ser como enunciamos no teorema anterior.

Teorema 4.2.4. Seja $I=(G, w)$ uma instância do $\mathrm{PCB}_{3}$ que consiste de um grafo 2-conexo $G=(V, E)$ e uma função $w: V \rightarrow \mathbb{Z}_{+}$. Seja $p_{\max }=\max \{w(v) \mid v \in V\}$. Então o algoritmo BalBicon 3 aplicado à instância I devolve uma 3-partição conexa de $G$ com medida $m$ tal que:

(a) Se $p_{\max } \geq \frac{1}{3} w(V)$ entâo $\frac{\operatorname{opt}(I)}{m} \leq \frac{4}{3}$.

(b) Se $p_{\max }<\frac{1}{3} w(V)$ então $\frac{\operatorname{opt}(I)}{m} \leq \frac{|V|}{3}$.

Agora vamos concentrar nossa atenção na classe de grafos 3-conexos. Como fizemos na Seção 3.3, podemos aqui explorar uma propriedade importante dos grafos 3-conexos. Conforme vimos no Lema 3.3.5, se $G$ é um grafo 3-conexo com pelo menos 5 vértices, então $G$ tem uma aresta $e$ tal que $G / e$ é 3-conexo.

Também é oportuno lembrarmos que dado um grafo $G$ e uma aresta $e$ de $G$, denotamos por $G / e$ o grafo obtido de $G$ pela contração da aresta $e$. Além disso, se $G$ é 3-conexo e $G / e$ é 3-conexo, então dizemos que a aresta $e$ é contraível.

Para simplificar a descrição do algoritmo $\mathrm{BalCon}_{3}$, descrevemos a seguir o procedimento Contraia-Aresta, que recebe como entrada um grafo 3-conexo e devolve um novo grafo que resulta da contração de uma aresta contraível desse grafo.

Procedimento Contraia-Aresta $(G, w)$

Entrada: um grafo 3-conexo $G=(V, E)$ e uma função $w: V \rightarrow \mathbb{Z}_{+}$.

Saída: um grafo 3-conexo $\widehat{G}=(\widehat{V}, \widehat{E})$ e uma função $\widehat{w}: \widehat{V} \rightarrow \mathbb{Z}_{+}$. $I^{*} \widehat{G}=G / e$, onde $e$ é uma aresta contraível de $G .{ }^{*} /$

1. Seja $e=(x, y)$ uma aresta contraível de $G$. 
2. Seja $\mathrm{Viz}=\{v \in V \mid v$ é adjacente a $x$ ou $y\} \backslash\{x, y\}$.

3. $\widehat{V}=V \backslash\{x, y\} \cup\{z\}$, onde $z \notin V$.

4. $\widehat{E}=E \backslash\{f \in E \mid f$ incidente a $x$ ou $y\} \cup\{z t \mid t \in \mathrm{Viz}\}$.

5. Seja $\widehat{G}=(\widehat{V}, \widehat{E})$.

6. Faça $\widehat{w}(v)=w(v)$ para todo $v \in \widehat{V} \backslash\{z\} ; \widehat{w}(z)=w(x)+w(y)$.

7. Devolva $(\widehat{G}, \widehat{w})$.

\section{fim_do_Procedimento}

Apresentamos a seguir a descrição formal do algoritmo $\mathrm{BalCon}_{3}$.

\section{Algoritmo $\mathrm{BalCon}_{3}(G, w)$}

Entrada: um grafo 3-conexo $G=(V, E)$ e uma função $w: V \rightarrow \mathbb{Z}_{+}$.

Saída: uma 3-partição conexa $(X, Y, \bar{Y})$ de $G$.

1. Seja $v_{1}=\arg \max \{w(v) \mid v \in V\}$.

2. $X=\left\{v_{1}\right\}$.

3. Se $w(X) \geq(1 / 3) w(V)$ então

3.1. Sejam $G^{\prime}=G-X$ e $w^{\prime}$ a restrição de $w$ aos vértices de $G^{\prime}$.

3.2. $(Y, \bar{Y})=\operatorname{BalBicon}_{2}\left(G^{\prime}, w^{\prime}\right)$.

3.3. Devolva $(X, Y, \bar{Y})$ e pare.

4. Se $w(X)<\frac{1}{6} w(V)$ então

4.1. $(\widehat{G}, \widehat{w})=(G, w) ; \widehat{V}=V$.

4.2. Enquanto não existe $\widehat{v} \in \widehat{V}$ tal que $\widehat{w}(\widehat{v}) \geq(1 / 6) \widehat{w}(\widehat{V})$ faça $(\widehat{G}, \widehat{w})=$ Contraia-Aresta $(\widehat{G}, \widehat{w})$.

4.3. $X=\{\widehat{v}\}$, onde $\widehat{w}(\widehat{v}) \geq(1 / 6) \widehat{w}(\widehat{V})$.

5. $G^{\prime}=\widehat{G}-X, w^{\prime}=\widehat{w}$.

6. $(Y, \bar{Y})=\operatorname{BalBicon}_{2}\left(G^{\prime}, w^{\prime}\right)$.

7. Se $X \neq\left\{v_{1}\right\}$ então reconstrua a partir de $(X, Y, \bar{Y})$ a 3 -partição $(X, Y, \bar{Y})$ de $G$.

8. Devolva $(X, Y, \bar{Y})$.

\section{fim_do_Algoritmo}


Provamos a seguir a razão de aproximação do BalCon $_{3}$.

Teorema 4.2.5. O algoritmo $\mathrm{BalCon}_{3}$ é uma 2-aproximação para o $\mathrm{PCB}_{3}$ restrito a grafos 3-conexos.

Prova: Seja $I=(G, w)$ uma instância do $\mathrm{PCB}_{3}$ que consiste de um grafo 3-conexo $G=(V, E)$ e uma função $w: V \rightarrow \mathbb{Z}_{+}$. Suponha que $v_{1}=$ $\arg \max \{w(v) \mid v \in V\}$. Seja $(X, Y, \bar{Y})$ a solução devolvida pelo algoritmo $\mathrm{BalCon}_{3}$ aplicado à instância $I$ e seja $m=\min \{w(X), w(Y), w(\bar{Y})\}$.

Inicialmente, note que pelo Lema 3.3.6 e pelo Teorema 3.3.1, a solução devolvida pelo $\mathrm{BalCon}_{3}$ é uma 3-partição conexa. Observe que, como o grafo $\widehat{G}$ ao final do passo 4.2 do $\mathrm{BalCon}_{3}$ é 3-conexo, então o grafo $G^{\prime}$ construído no passo 5 é 2-conexo. Logo, o algoritmo BalBicon 2 é usado apropriadamente no passo 6. Observe que o procedimento Contraia-Aresta pode ser sempre executado já que na hora da chamada desse procedimento o grafo $\widehat{G}$ é 3-conexo e tem pelo menos 6 vértices. Note também que a reconstrução mencionada no passo 7 pode ser de fato efetuada, conforme o Lema 3.3.6.

Todos os passos deste algoritmo podem ser implementados de modo que sua execução seja polinomial. De fato, a polinomialidade do BalBicon 2 usado nos passos 3.2 e 6 é comprovada pelo Teorema 3.3.1; o procedimento Contraia-Aresta (que é chamado no máximo $|E|$ vezes) pode ser implementado de modo que sua complexidade seja polinomial no tamanho do grafo; o passo 7 para reconstruir a 3-partição para o grafo original pode ser feito em tempo $O(|V|)$.

Agora, para mostrarmos a aproximação desejada, analisamos três casos.

CAso 1. Existe $v \in V$ tal que $w(v) \geq(1 / 3) w(V)$.

Neste caso, temos que a 3-partição $(X, Y, \bar{Y})$ foi devolvida no passo 3.3. Logo, pelo Teorema 4.2.2 a qualidade da solução é aquela garantida pelo algoritmo BalBicon $_{2}$ utilizado no Passo 3.2. Conseqüentemente, pelo Corolário 3.3.1 concluímos que

$$
\frac{\operatorname{opt}(I)}{m} \leq \frac{4}{3}
$$

CAso 2. Existe $v \in V$ tal que $(1 / 6) w(V) \leq w(v)<(1 / 3) w(V)$.

Neste caso temos que $X=\left\{v_{1}\right\}$ e $(1 / 6) w(V) \leq w(X)<(1 / 3) w(V)$. 
CASO 2.1. $m=w(X)$.

Então, $m \geq(1 / 6) w(V)$, já que $w(X) \geq(1 / 6) w(V)$, conforme vimos acima.

CASO 2.2. $m=\min \{w(Y), w(\bar{Y})\}$.

Neste caso, $(Y, \bar{Y})=\operatorname{BalBicon}_{2}\left(G^{\prime}, w^{\prime}\right)$, onde $G^{\prime}=\left(V^{\prime}, E^{\prime}\right)$ e $V^{\prime}=V \backslash X$.

Portanto, $w\left(V^{\prime}\right)>(2 / 3) w(V)$, visto que $w(X)<(1 / 3) w(V)$.

Como $w(v)<(1 / 3) w(V)$ para todo $v \in V^{\prime}$, temos que $w(v)<(1 / 2) w\left(V^{\prime}\right)$. Assim, pelo Teorema 3.3.2 temos que $m \geq(1 / 3) w\left(V^{\prime}\right)$. Logo, $m>$ $(1 / 3)(2 / 3) w(V)=(2 / 9) w(V)$.

Juntando os casos (2.1) e (2.2) temos que $m \geq(1 / 6) w(V)$. Portanto, $m \geq$ $(1 / 6) w(V)=(1 / 2)(w(V) / 3) \geq(1 / 2) \operatorname{opt}(I)$. Ou seja,

$$
\frac{\operatorname{opt}(I)}{m} \leq 2
$$

CASO 3. $w(v)<(1 / 6) w(V)$ para todo $v \in V$.

Pelo passo 4.3 do algoritmo, temos que $X=\{\widehat{v}\}$, onde $\widehat{w}(\widehat{v}) \geq(1 / 6) \widehat{w}(\widehat{V})$. Vamos considerar duas situações.

CASO 3.1. $m=w(X)$.

Neste caso, $m \geq(1 / 6) \widehat{w}(\widehat{V})=(1 / 6) w(V)$, pois ${ }^{1} \widehat{w}(\widehat{V})=w(V)$.

CASO 3.2. $m=\min \{w(Y), w(\bar{Y})\}$.

Note que, $X=\{\widehat{v}\}$ onde $\widehat{v}$ é o primeiro vértice de $\widehat{G}$ obtido pela contração de uma ou mais arestas tal que $\widehat{w}(\widehat{v}) \geq(1 / 6) \widehat{w}(\widehat{V})$. Assim, se $\widehat{v}$ resultou da contração de uma aresta $x y$, então

$$
\widehat{w}(\widehat{v})=\widehat{w}(x)+\widehat{w}(y)<(1 / 6) \widehat{w}(\widehat{V})+(1 / 6) \widehat{w}(\widehat{V})=(1 / 3) \widehat{w}(\widehat{V})=(1 / 3) w(V) .
$$

Como $G^{\prime}=G-X$, em $G^{\prime}$ temos que todos os vértices têm peso menor que $(1 / 6) w(V)$. Como $w\left(V^{\prime}\right)=w(V)-w(X)>(2 / 3) w(V)$, então esses vértices de $G^{\prime}$ têm peso menor que $(1 / 4) W\left(V^{\prime}\right)$. Logo, a 2-partição

\footnotetext{
${ }^{1}$ Observe que o procedimento Contraia-Aresta não altera a soma de pesos de todos os vértices do grafo. Em outras palavras, a cada iteração temos $\widehat{w}(\widehat{V})=w(V)$.
} 
conexa $(Y, \bar{Y})$ do grafo $G^{\prime}$ devolvida pelo algoritmo BalBicon 2 possui medida $m \geq(1 / 3) w\left(V^{\prime}\right)$, pelo Teorema 3.3.2. Portanto, $m \geq(1 / 3) w\left(V^{\prime}\right)>$ $(1 / 3)(2 / 3) w(V)=(2 / 9) w(V)$.

Juntando os casos (3.1) e (3.2) podemos concluir que no Caso 3 temos que $m \geq(1 / 6) w(V)$. Assim, analogamente ao Caso 2 concluímos que

$$
\frac{\operatorname{opt}(I)}{m} \leq 2
$$

Portanto, juntando os 3 casos, temos que $\frac{\mathrm{opt}(I)}{m} \leq 2$, conforme queríamos demonstrar.

Corolário 4.2.2. Seja I uma instância do $\mathrm{PCB}_{3}$ que consiste de um grafo 3conexo $G=(V, E)$ e uma função $w: V \rightarrow \mathbb{Z}_{+}$. Seja $v_{1}=\arg \max \{w(v) \mid v \in V\}$. Se $w\left(v_{1}\right)<(1 / 3) w(V)$, então o algoritmo BalCon ${ }_{3}$ aplicado a I devolve uma 3partição conexa de medida $m$ tal que $m \geq(1 / 6) w(V)$.

Mostramos na próxima seção como algumas das idéias vistas nesta seção podem ser utilizadas para desenvolver algoritmos para o $\mathrm{PCB}_{q}, q \geq 4$, restrito a grafos $q$-conexos.

\subsection{Generalização}

Na Seção 4.1 mencionamos que para grafos $q$-conexos sem pesos o $\mathrm{PCB}_{q}$ pode ser resolvido em tempo polinomial. Também vimos que para $q=2$ e $q=3 \mathrm{o}$ problema correspondente, na sua versão com pesos, é NP-difícil. (Vimos que a versão de decisão é um problema NP-completo.)

Provamos a seguir um resultado análogo para cada $q \geq 4, q$ fixo, pode ser provado por indução em $q$. A prova do passo indutivo é análoga à que fizemos para o caso $q=3$. Temos assim o seguinte resultado.

Teorema 4.3.1. Para cada natural $q \geq 2$, a versão de decisão do $\mathrm{PCB}_{q}$ restrito a grafos q-conexos é um problema NP-completo.

As idéias discutidas na Seção 4.2 podem ser utilizadas para obter uma aproximação para o $\mathrm{PCB}_{4}$ restrito a grafos 4-conexos. Na verdade, temos uma 2aproximação para esse problema, porém, apenas sob a hipótese de que o grafo de 
entrada tem pelo menos um vértice com peso pelo menos $\frac{1}{8} w(V)$. Para isso, usamos essencialmente o fato de que para o $\mathrm{PCB}_{3}$ restrito a grafos 3-conexos temos uma 2-aproximação.

A seguir, descrevemos uma generalização do algoritmo $\mathrm{BalCon}_{3}$, que denominamos de $\mathrm{BalCon}_{q}$, para $q \geq 3$ e grafos $q$-conexos. Notamos porém que o uso do algoritmo Hipo $_{q-1}$ no passo 6 requer que o mesmo seja um algoritmo para encontrar uma ( $q-1)$-partição num grafo 2-conexo. Assim, no caso $q=3$, podemos usar o BalBicon ${ }_{2}$ no lugar do $\mathrm{Hipo}_{2}$, obtendo exatamente o algoritmo BalCon 3 descrito anteriormente.

\section{Algoritmo $\mathrm{BalCon}_{q}(G, w)$}

Entrada: um grafo $q$-conexo $G=(V, E)$ e uma função $w: V \rightarrow \mathbb{Z}_{+}$.

Saída: uma $q$-partição conexa de $G$.

1. Seja $v_{1}=\arg \max \{w(v) \mid v \in V\}$.

2. $V_{1}=\left\{v_{1}\right\}$.

3. Se $w\left(V_{1}\right) \geq(1 / q) w(V)$ então

3.1. Sejam $G^{\prime}=G-V_{1}$ e $w^{\prime}$ a restrição de $w$ aos vértices de $G^{\prime}$.

3.2. $\left(V_{2}, \ldots, V_{q}\right)=\operatorname{BalCon}_{q-1}\left(G^{\prime}, w^{\prime}\right)$.

3.3. Devolva $\left(V_{1}, V_{2}, \ldots, V_{q}\right)$ e pare.

4. Se $w\left(V_{1}\right)<\frac{1}{2 q} w(V)$ então

4.1. $(\widehat{G}, \widehat{w})=(G, w) ; \widehat{V}=V$.

4.2. Enquanto não existe $\widehat{v} \in \widehat{V}$ tal que $\widehat{w}(\widehat{v}) \geq \frac{1}{2 q} \widehat{w}(\widehat{V})$ faça $(\widehat{G}, \widehat{w})=$ Contraia-Aresta $(\widehat{G}, \widehat{w})$.

4.3. $V_{1}=\{\widehat{v}\}$, onde $\widehat{w}(\widehat{v}) \geq \frac{1}{2 q} \widehat{w}(\widehat{V})$.

5. $G^{\prime}=\widehat{G}-V_{1}, w^{\prime}=\widehat{w}$.

6. $\left(V_{2}, \ldots, V_{q}\right)=\operatorname{Hipo}_{q-1}\left(G^{\prime}, w^{\prime}\right) \cdot{ }^{*} G^{\prime}$ é 2 -conexo $* /$

7. Se $V_{1} \neq\left\{v_{1}\right\}$ então reconstrua a partir de $\left(V_{1}, V_{2}, \ldots, V_{q}\right)$ a $q$-partição $\left(V_{1}, V_{2}, \ldots, V_{q}\right)$ de $G$.

8. Devolva $\left(V_{1}, V_{2}, \ldots, V_{q}\right)$.

\section{fim_do_Algoritmo}


Se o grafo de entrada tiver conectividade bem alta, é possível que o grafo $G^{\prime}$ construído no passo 5 seja 3-conexo. Sabemos que o grafo $\widehat{G}$ obtido no final do passo 4 é 3-conexo; e portanto $G^{\prime}$ é pelo menos 2-conexo.

Se tivéssemos um algoritmo $\mathrm{Hipo}_{q-1}$ que encontra para um grafo 2-conexo uma $(q-1)$-partição conexa com medida pelo menos $w(V) / \alpha$, poderíamos garantir que $\mathrm{BalCon}_{q}$ tem razão de aproximação $\max \left\{2, \frac{\alpha}{q-1}\right\}$.

No caso $q=4$ podemos usar o algoritmo $\mathrm{BalCon}_{3}$ no passo 3.2 e garantir que a solução devolvida no passo 3.3 é uma 2-aproximação. Se $w(v) \geq(1 / 8) w(V)$ para algum vértice $v$ então o passo 4 não é executado e a 3-partição devolvida no passo 6 - obtida usando o algoritmo $\mathrm{BalCon}_{3}$ - tem medida pelo menos $w\left(V^{\prime}\right) / 6$, e portanto pelo menos $w(V) / 8$. Com isso, garantimos uma 2-aproximação. Mais formalmente, temos o seguinte resultado.

Teorema 4.3.2. Seja I uma instância do $\mathrm{PCB}_{4}$ que consiste de um grafo 4-conexo $G=(V, E)$ e uma função $w: V \rightarrow \mathbb{Z}_{+}$tal que $w(v) \geq \frac{1}{8} w(V)$ para algum vértice v em $V$. Então o algoritmo $\mathrm{BalCon}_{4}$ aplicado a I devolve em tempo polinomial uma 4-partiçấo conexa com medida $m$ tal que:

$$
\frac{\operatorname{opt}(I)}{m} \leq 2 \text {. }
$$

Encerramos este capítulo observando que, para conseguirmos usar o algoritmo $\mathrm{BalCon}_{q}$ para $q \geq 5$, precisamos ter um algoritmo para obter $(q-1)$-partições de grafos 2-conexos com boa garantia, conforme observamos anteriormente. No momento, o algoritmo que descrevemos não nos permite obter resultados com boas garantias. Isto sugere uma possível direção para pesquisas futuras nessa linha. 



\title{
Problemas Correlatos
}

\author{
"Novamente diminuímos o número de questões \\ em aberto, sem contudo aumentar muito o \\ número de respostas." \\ (C. H. Papadimitriou e M. Yannakakis)
}

Os estudos realizados nos capítulos anteriores sobre o problema Max $q$-Partição Conexa Balanceada $\left(\mathrm{PCB}_{q}\right), q \geq 2$, motivaram-nos a investigar dois outros problemas correlatos: o problema Max Árvore Balanceada (ArvBal) e o problema $\operatorname{Max}(p / k)$-Bipartição Fracionária Conexa $\left(\mathrm{BFC}_{p / k}\right)$. Sobre o primeiro, encontramos várias referências na literatura [CGM83, CGM86, CGM80, GMM97], sendo o mesmo denominado Max Balance ou Max Balanced Tree. Quanto ao segundo, nós o formulamos como uma outra possível generalização do $\mathrm{PCB}_{2}$. Isto ficará claro assim que apresentarmos a definição deste problema.

Os estudos sobre esses dois problemas serão apresentados nas duas próximas seções. Na última seção deste capítulo discutimos outros problemas correlatos, mencionando os resultados encontrados na literatura.

\subsection{O problema Max Árvore Balanceada}

Problemas sobre árvores geradoras têm sido amplamente estudados. Uma das razões desse interesse é que vários problemas da área de projeto de redes 
são freqüentemente associados a árvores [CGM86, GMM97, Gar96, Aro98, FR94, CKST99, LR92, GMM94, WLB ${ }^{+99, ~ B P 89] . ~ M u i t o s ~ p r o b l e m a s ~ c o n s i s t e m ~ e m ~ d e s-~}$ cobrir - num dado grafo com pesos associados aos seus vértices ou arestas uma árvore geradora que satisfaz algum critério de otimalidade. Galbiati et. al [CGM80, CGM83, CGM86, GMM94, GMM97] estudaram a complexidade de vários problemas sobre árvores geradoras na tentativa de identificar o limite entre problemas fáceis e difíceis, bem como, investigar propriedades de aproximação de alguns deles.

O problema Max Árvore Balanceada (ArvBal) também pode ser visto sob este enfoque. Antes de apresentarmos a definição deste problema, lembramos que para simplificar, se $T$ é uma árvore, em vez de $w(V(T))$, escrevemos $w(T)$, a soma dos pesos dos vértices de $T$.

\section{Max Árvore Balanceada (ArvBal)}

Instância: um grafo conexo $G=(V, E)$ e uma função $w: V \rightarrow \mathbb{Z}_{+}$.

Solução viável: uma árvore geradora $T$ de $G$.

Função: $\max \{\mathcal{F}(e, T) \mid e \in E(T)\}$, onde $\mathcal{F}(e, T)=w\left(T_{1}\right) \cdot w\left(T_{2}\right)$, e $T_{1}, T_{2}$ são as árvores de $T-e$.

Objetivo: maximizar.

A versão sem pesos do problema Max Árvore Balanceada é equivalente ao de descobrir em $G$ uma árvore geradora $T$ e uma aresta $e$ de $T$ tal que o número de caminhos em $T$ conectando pares de vértices de $T$ que passam pela aresta $e$ seja máximo.

Note que as soluções ótimas de ArvBal e $\mathrm{PCB}_{2}$ estão intimamente relacionadas. De fato, suponha que $(G, w)$ seja uma instância de ambos os problemas. Se $T$ é uma solução dessa instância para o ArvBal, então para qualquer aresta $e$ de $T$, se $T-e=T_{1} \cup T_{2}$, temos que $\left(V\left(T_{1}\right), V\left(T_{2}\right)\right)$ é uma solução para o $\mathrm{PCB}_{2}$. Por outro lado, se $\left(V_{1}, V_{2}\right)$ é uma partição conexa de $G$, então podemos obter uma árvore geradora $T$ de $G$ e uma aresta $e$ em $T$ tal que $\mathcal{F}(e, T)=w\left(T_{1}\right) \cdot w\left(T_{2}\right)=$ $w\left(V_{1}\right) \cdot w\left(V_{2}\right)$. Para isso, basta tomar $T=T_{1} \cup T_{2}+e$ onde $T_{i}$ é uma árvore geradora do subgrafo induzido por $V_{i}, 1 \leq i \leq 2$, e $e$ é uma aresta de $G$ com um extremo em $V_{1}$ e outro em $V_{2}$.

Como para cada aresta $e$ de uma árvore geradora $T$ tal que $T-e=T_{1} \cup T_{2}$, temos que $w\left(T_{2}\right)=w(V)-w\left(T_{1}\right)$ e a função $\mathcal{F}(e, T)=w\left(T_{1}\right) \cdot w\left(T_{2}\right)$ cresce quando $w\left(T_{1}\right)$ 
cresce e atinge seu máximo quando $w\left(T_{1}\right)$ é máximo (sujeito a $w\left(T_{1}\right) \leq w(V) / 2$ ), segue imediatamente que tendo uma solução ótima de um dos problemas obtemos uma solução ótima do outro.

Logo, todos os resultados conhecidos na literatura e aqueles provados nesta tese a respeito da complexidade do $\mathrm{PCB}_{2}$ podem ser estendidos para ArvBal, tanto os casos polinomiais quanto NP-completos.

De acordo com as observações anteriores podemos também concluir que resultados análogos ao Teorema 3.2.2 e o Corolário 3.2.1 sobre inaproximabilidade do $\mathrm{PCB}_{2}$ valem também para o Arvbal.

Para grafos conexos quaisquer, o melhor resultado é o algoritmo 1.072aproximado obtido por Chlebíková [Chl96]. Este resultado pode ser obtido do próprio algoritmo $\mathrm{BalCon}_{2}$ fazendo uma análise para o ArvBal análoga à que fizemos para garantir uma (4/3)-aproximação para o $\mathrm{PCB}_{2}$. Com relação à existência de esquema de aproximação polinomial podemos afirmar que a resposta é positiva para as mesmas classes de grafos solucionadas no Capítulo 3. Isto segue do Teorema 2.3.1 e do seguinte resultado sobre AP-redução.

Teorema 5.1.1. Max Árvore Balanceada $\leq_{A P}^{1}$ Max 2-Partição Conexa Balanceada.

Prova: Para simplificar a notação vamos chamar de $A$ o problema ArvBal e $B$ o problema $\mathrm{PCB}_{2}$. Afirmamos que a tripla $(f, g, \alpha)$ definida abaixo é uma APredução entre os problemas $A$ e $B$.

1. Para qualquer $x \in I_{A}$, e para qualquer racional $r>1$, seja $f(x, r)=x$.

2. Para qualquer $x=(G, w) \in I_{A}$, para qualquer $y=\left(V_{1}, V_{2}\right) \in \operatorname{sol}_{B}(x) \mathrm{e}$ para qualquer $r>1$, seja $g(x, y, r)=T_{y}$, onde $T_{y}$ é a árvore geradora de $G$ associada à solução $y$, conforme explicamos anteriormente.

3. $\alpha=1$.

É fácil verificar que as funções $f$ e $g$ satisfazem as condições exigidas para uma AP-reducibilidade. Resta-nos provar que para qualquer $x \in I_{A}$, para qualquer $r>1$ e para qualquer $y \in \operatorname{sol}_{B}(x)$,

$$
\text { se } R_{B}(x, y) \leq r \text { então } R_{A}\left(x, T_{y}\right) \leq r .
$$


Em vista das discussões feitas anteriormente sobre a construção de $T_{y}$, é imediato que para qualquer instância $x=(G, w) \in I_{A}$ e para qualquer $y \in \operatorname{sol}_{B}(x)$ é válida a seguinte igualdade, onde $W=w(V(G))$ :

$$
m_{A}\left(x, T_{y}\right)=m_{B}(x, y)\left(W-m_{B}(x, y)\right) .
$$

Suponha que para tal $x$ e tal $y$ temos que $R_{B}(x, y) \leq r$. Então,

$$
R_{A}\left(x, T_{y}\right)=\frac{o p t_{A}(x)}{m_{A}(x, y)}=\frac{\operatorname{opt}_{B}(x)\left(W-o p t_{B}(x)\right)}{m_{B}(x, y)\left(W-m_{B}(x, y)\right)} .
$$

Visto que $W \geq \operatorname{opt}_{B}(x) \geq m_{B}(x, y)$, temos que $W-o p t_{B}(x) \leq W-m_{B}(x, y)$. Substituindo esta desigualdade na fórmula acima, obtemos o resultado que desejamos,

$$
R_{A}\left(x, T_{y}\right) \leq \frac{o p t_{B}(x)}{m_{B}(x, y)} \leq r .
$$

\subsection{Bipartição Fracionária Conexa}

Como afirmamos anteriormente, o problema que definimos a seguir pode ser visto como uma outra maneira de se generalizar o $\mathrm{PCB}_{2}$.

\section{Max Bipartição Fracionária Conexa $\left(\mathrm{BFC}_{p / k}\right)$}

Instância: um grafo conexo $G=(V, E)$ e uma função $w: V \rightarrow \mathbb{Z}_{+}$.

Solução viável: uma 2-partição conexa $\left(V_{1}, V_{2}\right)$ de $G$ tal que $w\left(V_{1}\right) \leq(p / k) w(V)$. Função: $w\left(V_{1}\right)$.

Objetivo: maximizar.

Definimos o problema $\mathrm{BFC}_{p / k}$ para inteiros $p$ e $k$ tais que $1 \leq p<k$. Note que $\mathrm{BFC}_{1 / 2}=\mathrm{PCB}_{2}$. É nesse sentido que o $\mathrm{BFC}_{p / k}$ pode ser visto como uma generalização do $\mathrm{PCB}_{2}$. É claro que aqui o conceito de balanceamento não existe, apenas o de obter uma bipartição onde uma das partes tenha peso tão próximo quanto possível de uma fração pré-estabelecida do peso total do grafo de entrada. 
Descrevemos a seguir um algoritmo para o $\mathrm{BFC}_{p / k}$, onde $p / k \geq 1 / 2$, e o grafo de entrada é 2-conexo.

Algoritmo $\mathrm{BFBiCon}(G, w, p, k)$

/* Estamos supondo que $1 / 2 \leq p / k<1$. */

Entrada: um grafo 2-conexo $G$ e uma função peso $w: V \rightarrow \mathbb{Z}_{+}$.

Saída: uma 2-partição conexa $\left(V_{1}, V_{2}\right)$ de $G$ tal que $w\left(V_{1}\right) \leq(p / k) w(V)$.

1. Sejam $v_{1}=\arg \max \{w(v) \mid v \in V\}$ e $\beta=(p / k) w(V)$.

2. Se $w\left(v_{1}\right)>\beta$ então $V_{1}=V \backslash\left\{v_{1}\right\}$; devolva $V_{1}$ e pare.

3. $V_{1}=\left\{v_{1}\right\}$.

4. Enquanto $w\left(V_{1}\right)<\beta$ faça

4.1 Escolha $u \in V_{2}$ tal que $u$ é admissível e de peso mínimo.

4.2 Se $w(u) \geq 2\left(\beta-w\left(V_{1}\right)\right)$ então $/ *$ CASO A : $w\left(V_{1}\right) \geq \beta-\frac{1}{2} w(u)$. */ break.

4.3 Senão $/ *$ CAso B */ $V_{1}=V_{1} \cup\{u\}$ e $V_{2}=V_{2} \backslash\{u\}$. $/^{*}$ Aqui temos $w\left(V_{1}\right)<\beta+\frac{1}{2} w(u)$. $/$

5. Se $w\left(V_{1}\right)>\beta$ então faça $V_{1}=V_{1} \backslash\{u\}$. $/ *$ Neste caso, $w\left(V_{1}\right)>\beta-w(u)$. */

6. Devolva $V_{1}$.

\section{fim_do_Algoritmo}

Provaremos a seguir o seguinte resultado a respeito do algoritmo BFBiCon.

Teorema 5.2.1. Sejam $p, k$ inteiros tais que $1 / 2 \leq p / k<1$. Seja I uma instância do $\mathrm{BFC}_{p / k}$ que consiste de um grafo 2-conexo $G=(V, E)$ e uma função $w: V \rightarrow \mathbb{Z}_{+}$. Seja $V=\left\{v_{1}, v_{2}, \ldots, v_{n}\right\}, n \geq 3, w\left(v_{1}\right) \geq w\left(v_{2}\right) \geq \ldots \geq w\left(v_{n}\right) e$ $t:=w(V) / w\left(v_{3}\right)$. Então o algoritmo BFBicon aplicado a $(G, w, p, k)$ devolve uma bipartição fracionária conexa $\left(V_{1}, V_{2}\right)$ de $G$ cuja medida $m=w\left(V_{1}\right)$ é tal que

$$
\frac{\operatorname{opt}(I)}{m} \leq \frac{p t}{p t-k}
$$

Ademais, o algoritmo BFBicon pode ser implementado de modo que ele fique com complexidade polinomial. 
Prova: A polinomialidade do algoritmo BFBicon segue da polinomialidade do algoritmo BalBicon 2 garantida pelo Teorema 3.3.1. Também é imediato que a solução devolvida é uma bipartição conexa de $G$.

Quanto à razão de aproximação deste algoritmo, primeiramente observamos que a solução devolvida no passo 2 é claramente ótima. Isto também ocorre quando $w\left(V_{1}\right)=\beta$ e o passo 4 não é executado. Vamos então analisar os dois casos que podem ocorrer (CAso A ou CASO B) antes da devolução da solução $V_{1}$.

CASO A: $w\left(V_{1}\right) \geq \beta-\frac{1}{2} w(u)$.

Neste caso, temos que $m=w\left(V_{1}\right) \geq(p / k) w(V)-(1 / 2)(w(V) / t)$, já que $w(u) \leq$ $w\left(v_{3}\right)$ e $t:=w(V) / w\left(v_{3}\right)$. Logo, $w\left(V_{1}\right) \geq\left(\frac{p}{k}-\frac{1}{2 t}\right) w(V)$.

CAso B: $\beta \leq w\left(V_{1}\right)<\beta+\frac{1}{2} w(u)$.

Neste caso, no passo 5 , se $w\left(V_{1}\right)=\beta$ temos que $V_{1}$ é uma solução ótima. Caso contrário, como fazemos $V_{1}=V_{1} \backslash\{u\}$, temos que $w\left(V_{1}\right)>\beta-w(u)$. Portanto, $m=w\left(V_{1}\right)>(p / k) w(V)-(1 / t) w(V)$ já que $w(u) \leq w\left(v_{3}\right)=(1 / t) w(V)$. Notamos aqui que $1 / t<p / k$. De fato, como $w\left(v_{1}\right)<(p / k) w(V)$, temos que $(1 / t) w(V)=$ $w\left(v_{3}\right)<(p / k) w(V)$. Logo,

Dos casos A e B deduzimos que $m=w\left(V_{1}\right)>\left(\frac{p}{k}-\frac{1}{t}\right) w(V)=\left(\frac{p t-k}{k t}\right) w(V)$.

$$
\operatorname{opt}(I) \leq \frac{p}{k} w(V)<\left(\frac{p}{k}\right) \frac{k t}{p t-k} m=\left(\frac{p t}{p t-k}\right) m
$$

Do teorema acima segue imediatamente o seguinte corolário (obtido do fato de que $t \geq 3$ ).

Corolário 5.2.1. Para inteiros $p$ e $k$ tais que $1 / 2 \leq p / k<1$, o algoritmo BFBicon é uma $\frac{3 p}{3 p-k}$-aproximação para o $\mathrm{BFC}_{p / k}$, restrito a grafos 2 -conexos.

Observamos que para frações $p / k$ tais que $1 / 3<p / k<1 / 2$ também podemos obter uma 3-aproximação para o $\mathrm{BFC}_{p / k}$. Deixamos a cargo do leitor a descrição de um algoritmo com essa razão de aproximação. Para as demais frações $p / k$, sabemos obter tal razão apenas sob a hipótese de que os vértices têm pesos no máximo $p / k$. 
Ressaltamos também que o algoritmo BFBicon requer que o grafo de entrada seja 2-conexo. Podemos porém, proceder como no caso do algoritmo $\mathrm{BalCon}_{2}$, que usa a idéia do grafo de blocos de $G$, e obter analogamente um algoritmo correspondente para grafos conexos. Como este não é o tema central deste trabalho, não iremos estender mais as discussões sobre o tópico desta seção.

\subsection{Outros problemas sobre partições conexas}

O tema central abordado nesta tese diz respeito a partições conexas balanceadas. O termo "partição balanceada" foi usado no sentido de designar uma partição cujas partes têm os pesos tão próximos (uniformes) quanto possível. Porém, a maneira de se medir essa proximidade (esse balanceamento), pode variar bastante, dando origem a diversos problemas, também conhecidos como problemas de equipartição.

Para apresentar esses problemas sob um mesmo enfoque, vamos considerar que a instância de todos esses problemas, tal como no $\mathrm{BCP}_{q}$, consiste de um grafo conexo $G=(V, E)$ e uma função $w: V \rightarrow \mathbb{Z}_{+}$, e que a solução viável é uma $q$-partição conexa $\left(V_{1}, V_{2}, \ldots, V_{q}\right)$ de $G$. Quanto à função objetivo, na sua forma geral, vamos considerar como sendo do seguinte tipo:

$$
\min _{p \in P(G, q)} g(p),
$$

onde $P(G, q)$ é o conjunto das $q$-partições conexas de $G$ e $g$ é uma função que mede a distância entre um elemento de $P(G, q)$ e uma partição, digamos ideal. Esta distância pode ser medida através de diferentes normas. Se $\psi=(1 / q) w(V)$, podemos utilizar:

- Norma $L_{1}: g(p)=\sum_{i=1}^{q}\left|w\left(V_{i}\right)-\psi\right|$.

- Norma $L_{2}: g(p)=\sum_{i=1}^{q}\left(w\left(V_{i}\right)-\psi\right)^{2}$.

- Norma $L_{\infty}: g(p)=\max _{i=1, \ldots, q}\left|w\left(V_{i}\right)-\psi\right|$.

Também é comum usar as funções:

(1) $\min \max \left\{w\left(V_{i}\right) \mid i=1, \ldots q\right\}$ ou

(2) $\max \min \left\{w\left(V_{i}\right) \mid i=1, \ldots q\right\}$. 
Note que no caso da função (1), temos que $g(p)=\max \left\{w\left(V_{i}\right) \mid i=1, \ldots q\right\}$. No caso da função (2) temos exatamente o $\mathrm{PCB}_{q}$.

Outros problemas de equipartição podem ser definidos onde a diferença entre os pesos máximo e mínimo de um conjunto de uma partição conexa é minimizada. Estes problemas ${ }^{1}$ são referenciados normalmente como 'particionamento mais uniforme' (PMU).

Ao mencionarmos esta variedade de funções objetivo, lembramos que apesar de esses problemas todos buscarem uma partição balanceada, estes podem ter propriedades de aproximação bem distintas.

Mesmo em termos de complexidade computacional, podemos também ter algumas diferenças interessantes. Por exemplo, todos os problemas desta classe são NP-difíceis [BLLS98] para grades $G_{m \times n} \operatorname{com} n \geq 3$. Contudo, se $g$ é a norma $L_{1}$, o problema de otimização associado é NP-difícil para árvores em geral e polinomial para casos especiais como estrelas, caminhos e lagartas (caterpillars) [AS75, SLPS90]. Algoritmos polinomiais que resolvem o problema da equipartição se $G$ é uma árvore e a função objetivo é dada por (1) e (2) são também conhecidos [BP83, BPS82, PS81]. O caso em que $G$ é um caminho também foi estudado e algoritmos polinomiais foram desenvolvidos para as funções objetivo (1) e (2) [CMNS99]. A mesma eficiência é obtida quando consideramos o problema PMU [LPS93] sobre um caminho, enquanto o caso geral é NP-difícil [GJ79]. Outro fato importante é que embora o $\mathrm{PCB}_{q}$ possa ser resolvido em tempo polinomial para escadas [BLLS01], este tempo polinomial pode não ser obtido para outras funções objetivo, a menos que $P=N P$. Por exemplo, para a norma $L_{1}$ e escadas foi provado que o problema da equipartição é NP-difícil [Lar94].

Como já mencionamos o $\mathrm{PCB}_{2}$ corresponde ao problema com a função (2). Assim, uma pergunta natural que surge é se os problemas definidos pelas funções (1) e (2) são equivalentes. É fácil verificar que estes problemas são equivalentes no caso $q=2$; e que para $q \geq 3$ isto não ocorre. Para mostrar este último fato, exibimos na Figura 5.1 uma instância para $q=4$. Considere o grafo dessa figura e a seguinte função $w$ definida sobre seus vértices: $w\left(v_{1}\right)=9, w\left(v_{2}\right)=7, w\left(v_{3}\right)=1$, $w\left(v_{4}\right)=3, w\left(v_{5}\right)=9, w\left(v_{6}\right)=12$ e $w\left(v_{7}\right)=8$. Observe que uma solução ótima para o $\mathrm{PCB}_{4}$ é a 4-partição conexa definida por $V_{1}=\left\{v_{1}, v_{2}\right\}, V_{2}=\left\{v_{3}, v_{5}\right\}$, $V_{3}=\left\{v_{6}\right\}$ e $V_{4}=\left\{v_{4}, v_{7}\right\}$, cuja medida é 10. Contudo, uma solução ótima para o

\footnotetext{
${ }^{1} \mathrm{O}$ termo original é MUP ou most uniform partitioning.
} 
problema definido pela função objetivo (1) é a seguinte: $V_{1}=\left\{v_{1}\right\}, V_{2}=\left\{v_{2}, v_{7}\right\}$, $V_{3}=\left\{v_{3}, v_{4}, v_{5}\right\}$ e $V_{4}=\left\{v_{6}\right\}$, cuja medida é 15 .

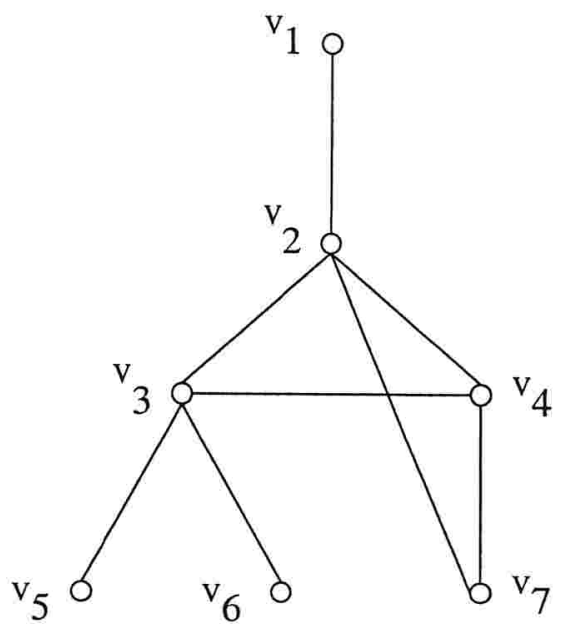

Figura 5.1: Uma instância com soluções distintas para as funções (1) e (2).

Neste capítulo, estudamos alguns problemas relacionados ao $\mathrm{PCB}_{q}, q \geq 2$. No que segue, apresentamos nossas considerações finais sobre o trabalho desenvolvido nesta tese. 



\title{
Considerações Finais
}

\author{
"Ter um ideal é o único meio de fazer algo e de \\ chegar a ser alguém. Só se compreende o que \\ a vida vale, quando a gente se coloca a serviço \\ de um ideal; pois a vida é triste se não é vivida \\ com uma ilusão." \\ (Afonso Milagro)
}

A aproximabilidade de problemas de otimização NP é um tema de grande interesse na área de otimização combinatória. Esta tese responde várias questões relacionadas com a complexidade computacional e as propriedades de aproximação de alguns problemas de partição de grafos em subgrafos conexos.

Um aspecto importante do nosso trabalho foi reunir os resultados existentes na literatura sobre problemas de partições conexas de grafos, investigar como estes se relacionam, propor novos problemas e novos algoritmos. Muitos problemas equivalentes são citados na literatura com nomes distintos, e alguns destes nem se referenciam uns aos outros. Mencionamos neste trabalho problemas que ocorrem sob nomes como maximally balanced connected partition [Chl96], max-min partitioning [PS81, BP95, BLLS01, BLLS98], equipartion [Lar94, AS75, SLPS90], max balance [GMM97], e outros.

Também contribuímos ao estudo da intratabilidade dos problemas estudados, provando alguns resultados nessa linha para algumas classes de grafos.

A partir da nossa releitura dos algoritmos apresentados por Chlebíková [Chl96] 
pudemos desenvolver algoritmos para o problema Max q-partição Conexa Balanceada $\left(\mathrm{PCB}_{q}\right)$, para $q \geq 3$. Da experiência obtida com nossas investigações, observamos que esta estratégia baseada em vértices admissíveis é um tanto limitada por não explorar a estrutura do grafo de entrada e utilizar sempre como limitante para o balanceamento ótimo o valor $w(V) / q$. Há muitas instâncias para as quais o valor ótimo está bem distante deste valor tomado como referência, e portanto, sem explorar mais a estrutura do grafo e a distribuição dos pesos, fica difícil conseguir algoritmos com boas garantias. É até surpreendente que ainda assim em alguns casos seja possível obter razões de aproximação relativamente boas.

Ressaltamos que a estratégia baseada em contrações de arestas que apresentamos para tratar grafos 3-conexos é inovadora. Obtivemos com essa estratégia um algoritmo para o $\mathrm{PCB}_{2}$ e um outro para o $\mathrm{PCB}_{3}$, este último, com razão de aproximação 2. Para o caso da 3-partição este é um dos primeiros na literatura para grafos mais gerais (o caso de árvores ou escadas já foi investigado). Para o problema $\mathrm{PCB}_{4}$ os resultados ainda são parciais, mas acreditamos que ainda possam ser melhorados.

Nossos estudos evidenciaram a importância de se obter bons resultados para grafos 2-conexos, para podermos obter bons algoritmos para o $\mathrm{PCB}_{q}$, quando $q \geq 3$. Observamos no Capítulo 4 que se obtivermos boas soluções aproximadas para o $\mathrm{PCB}_{3}$ e o $\mathrm{PCB}_{4}$ isto terá impacto direto no algoritmo generalizado que propomos para o $\mathrm{PCB}_{q}$, eventualmente apenas com a hipótese de 3-conexidade.

Nesta tese concentramos esforços investigando a questão da existência de um PTAS para o $\mathrm{PCB}_{2}$. Consideramos essa questão de grande interesse. Como frutos desta investigação obtivemos um esquema de aproximação para uma classe especial de grafos, bem como, mostramos um resultado importante de inaproximabilidade. Continuar investigando esta questão é uma boa sugestão para trabalhos futuros. 


\section{Referências Bibliográficas}

[AC99] P. Alimonti and T. Calamoneri. "On the complexity of the max balance problem". In Argentinian Workshop on Theoretical Computer Science (WAIT'99), pages 133 - 138, 1999.

[ACG ${ }^{+99}$ G. Ausiello, P. Crescenzi, G. Gambosi, V. Kann, A. MarchettSpaccamela, and M. Protasi. Complexity and Approximation. Combinatorial Optimization Problems and Their Approximability Properties. Springer-Verlag, 1999.

[ADP80] G. Ausiello, A. D'Atri, and M. Protasi. "Structure preserving reductions among convex optimization problems". Journal of Computer and System Sciences, 21(1):136-153, 1980.

[AKR95] A. Agrawal, P. Klein, and R. Ravi. "When trees collide: an approximation algorithm for the generalized Steiner problem on networks". SIAM Journal on Computing, 24(3):440 - 456, 1995.

[ALM+98] S. Arora, C. Lund, R. Motwani, M. Sudan, and M. Szegedy. "Proof verification and the hardness of approximation problems". Journal of the ACM, 45(3):501 - 555, 1998.

[Aro98] S. Arora. "Polynomial time approximation scheme for Euclidean TSP and other geometric problems". Journal of the ACM, 45(5):753 $-782,1998$.

[AS73] E. Aparo and B. Simeone. "Un algoritmo di equipartizione e il suo 
impiego in un problema de contrasto ottico". Ricerca Operativa, 3:31 $-42,1973$.

[AS75] E. Aparo and B. Simeone. "Equipartizione su un grafo: Un'applicazione all'informatica medica". In Applicazioni del Calcolo, pages 77 -86, Roma, 1975.

[AS98] S. Arora and S. Safra. "Probabilistic checking of proofs: a new characterization of NP". Journal of the ACM, 45(1):70 - 122, 1998.

[Bab85] L. Babai. "Trading group theory for randomness". In Proceedings of the 17th Annual ACM Symposium on Theory of Computing, pages $421-429,1985$.

[BCG93] T. Beherendt, K. Compton, and E. Grädel. "Optimization problems: expressibility, approximation properties and expected asymptotic growth of optimal solutions". In Proceedings of the 6th Workshop of Computer Science and Logic, volume 702 of Lectures Notes in Computer Science, pages 43 -60. Springer - Verlag, 1993.

[Bel93] M. Bellare. "Interactive proofs and approximation: reductions from two provers in one round". In Proceedings of the 2nd Israel Symposium on Theory of Computing and Systems. IEEE, 1993.

[BFL91] L. Babai, L. Fortnow, and C. Lund. "Non-deterministic exponential time has two-prover interactive protocols". Computational Complexity, 1:3 - 40, 1991.

[BFLS91] L. Babai, L. Fortnow, L. Levin, and M. Szegédy. "Checking computations in polylogarithmic time". In Proceedings of the 23rd Annual ACM Symposium on Theory of Computing, pages 21 - 31, 1991.

[BGS98] M. Bellare, O. Goldreich, and M. Sudan. "Free bits, PCPs and non-approximability-towards tight results". SIAM Journal on Computing, 27(3):804-915, 1998.

[BH92] R. Boppana and M. Halldórsson. "Approximating maximum independent sets by excluding subgraphs". BIT, 32(2):180 - 196, 1992. 
[BLLS98] R. Becker, I. Lari, M. Lucertini, and B. Simeone. "Max-min partitioning of grid graphs into connected components". Networks, 32(2):115 - 125, 1998.

[BLLS01] R. Becker, I. Lari, M. Lucertini, and B. Simeone. "A polynomial-time algorithm for max-min partitioning of ladders". Theory of Computing Systems, 34(4):353 - 374, 2001.

[BM88] L. Babai and S. Moran. "Arthur-Merlin games: a randomized proof system, and a hierarchy of complexity classes". Journal of Computer and System Sciences, 36(2):254 - 276, 1988.

[BOGKW90] M. Ben-Or, S. Goldwasser, J. Kilian, and A. Wigderson. "Efficient identification schemes using two prover interactive proofs". In Proceedings of Advance in Cryptology - Crypto'89, volume 435 of Lecture Notes in Computer Science, pages 498 - 506. Springer, 1990.

[BP83] R. Becker and Y. Perl. "Shifting algorithm for tree partitioning with general weighting functions". Jornal of Algorithms, 4(2):101 - 120, 1983.

[BP89] M. Bern and P. Plassmann. "The steiner problem with edge lengths 1 and 2". Information Processing Letters, 32:171 - 176, 1989.

[BP95] R. Becker and Y. Perl. "The shifting algorithm technique for the partitioning of trees". Discrete Applied Mathematics, 62:15 - 34, 1995.

[BPS82] R. Becker, Y. Perl, and S. Schach. "A shifting algorithm for min-max tree partitioning". Journal of the ACM, 29(1):58 - 67, 1982.

[BS92] P. Berman and G. Schnitger. "On the complexity of approximating the independent set problem.". Information and Computation, 96(1):77 - 94, 1992.

[BS94] M. Bellare and M. Sudan. "Improved non-approximability results". In Proceedings of the 26th Annual ACM Symposium on Theory of Computing, pages 184 - 193, 1994.

[CGL97] R. Chang, W. Gasarch, and C. Lund. "On bounded queries and approximation". SIAM Journal on Computing, 26(1):188 - 209, 1997. 
[CGM80] P. Camerini, G. Galbiati, and F. Maffioli. "Complexity of spanning tree problems: Part I". European Journal of Operational Research, $5: 346-352,1980$.

[CGM83] P. Camerini, G. Galbiati, and F. Maffioli. "On the complexity of finding multi-constrained spanning trees". Discrete Applied Mathematics, 5:39-50, 1983.

[CGM86] P. Camerini, G. Galbiati, and F. Maffioli. "The complexity of weighted multi-constrained spanning tree problems". Colloquia Mathematica Societais Janos Bolyai, 44:53-101, 1986.

[Cha96] R. Chang. "On the query complexity of clique size and maximum satisfiability". Journal of Computer and System Sciences, 53(2):298 - 313, 1996.

[Chl96] J. Chlebíková. "Approximating the maximally balanced connected partition problem in graphs". Information Processing Letters, 60:225 - 230, 1996.

[Chv79] V. Chvátal. "A greedy heuristic for the set-covering problem". Mathematics of Operations Research, 4(3):233 - 235, 1979.

[CK98] P. Crescenzi and V. Kann. "How to find the best approximation results: a follow-up to Garey and Johnson". ACM SIGACT News, 29(4):90 - 97, 1998.

[CKST99] P. Crescenzi, V. Kann, R. Silvestri, and L. Trevisan. "Structure in approximation classes". SIAM Journal on Computing, 28:17591782, 1999 .

[CMNS99] F. Conti, F. Malucelli, S. Nicoloso, and B. Simeone. "On a 2dimensional equipartition problem". European Journal of Operational Research, 113(1):215 - 231, 1999.

[Coo71] S. A. Cook. "The complexity of theorem-proving procedures". In Proceedings of the 3rd. Annual ACM Symposium on Theory of Computing, pages $151-158,1971$.

[CP91] P. Crescenzi and A. Panconesi. "Completeness in approximation classes". Information and Computation, 93(2):241 - 262, 1991. 
[CST01] P. Crescenzi, R. Silvestri, and L. Trevisan. "On weight vs unweighted versions of combinatorial optimization problems". Information and Computation, 167(1):10 - 26, 2001.

[CT00] P. Crescenzi and L. Trevisan. "On approximation scheme preserving reducibility and its applications". Theory of Computing Systems, 33(1):1 - 16, 2000.

[DF85] M. Dyer and A. Frieze. "On the complexity of partitioning graphs into connected subgraphs". Discrete Applied Mathematics, 10:139153, 1985.

[DFF56] G. Dantzig, L. Ford, and D. Fulkerson. "A primal-dual algorithm for linear programs". In H. W. Kuhn and A. W. Tucker, editors, Linear Inequalities and Related Systems, pages $171-181$. Princeton University Press, Princeton, NJ, 1956.

[Die00] R. Diestel. Graph theory, volume 173 of Graduate Texts in Mathematics. Springer-Verlag, New York, second edition, 2000.

[Diw03] A. Diwan. "Partitioning 2-connected graphs into connected subgraphs". Submitted, 2003.

[Fag74] R. Fagin. "Generalized first-order spectra and polynomial-time recognizable sets". In Proceedings of the Complexity of Computations, volume 7, pages 43 - 73. SIAM - AMS, 1974.

$\left[\mathrm{FGL}^{+} 96\right] \quad$ U. Feige, S. Goldwasser, L. Lovász, S. Safra, and M. Szegédy. "Interactive proofs and the hardness of approximating cliques". Journal of the ACM, 43(2):268 - 292, 1996.

[FL92] U. Feige and L. Lovász. "Two-prover one round proof systems: their power and their problems". In Proceedings of the 24th Annual ACM Symposium on Theory of Computing, pages 733 - 744, 1992.

[FR94] M. Fürer and B. Raghavachari. "Approximating the minimum degree steiner tree to within one of optimal". Journal of Algorithms, 17:409 - 423, 1994. 
[FRS94] L. Fortnow, J. Rompel, and M. Sipser. "On the power of multi-prover interactive protocols". Theoretical Computer Science A, 134:545557, 1994.

[Gar96] N. Garg. "A 3-approximation for the minimum tree spanning $k$ vertices". In Proceeding of the 37th Annual IEEE Symposium on Foundations of Computer Science, pages 302 - 309, 1996.

[GJ76] M. Garey and D. Johnson. "The complexity of near-optimal graph coloring". Journal of the ACM, 23:43-49, 1976.

[GJ78] M. Garey and D. Johnson. "Strong NP-completeness results: motivations, examples and implications". Journal of the ACM, 25:499508, 1978.

[GJ79] M. Garey and D. Johnson. Computers and Intractability: a Guide to the Theory of NP-Completeness. W. H. Freeman and Company, San Francisco, 1979.

[GMM94] G. Galbiati, F. Maffioli, and A. Morzenti. "A short note on the approximability of the maximum leaves spanning tree problem". Information Processing Letters, 52:45 - 49, 1994.

[GMM95] G. Galbiati, F. Maffioli, and A. Morzenti. "On the approximability of some maximum spanning tree problems". In Second International Symposium of Latin American Theoretical INformatics, volume 911 of Lecture Notes in Computer Science, pages 300 - 310, SpringVerlag,Berlin, 1995.

[GMM97] G. Galbiati, F. Maffioli, and A. Morzenti. "On the approximability of some maximum spanning tree problems". Theoretical Computer Science, 181(1):107 - 118, 1997.

[GMR89] S. Goldwasser, S. Micali, and C. Rackoff. "The knowledge complexity of interactive proof systems". SIAM Journal on Computing, 18(1):186 - 208, 1989.

[Gra66] R. Graham. "Bounds for certain multiprocessing anomalies". Bell System Technology Journal, 45:1563 - 1581, 1966. 
[GW94] M. Goemans and D. Williamson. "0.878-approximation algorithms for max cut and max 2sat". In Proceedings of the 26th Annual ACM Symposium on Theory of Computing, pages 422 - 431, 1994.

[GW95a] M. Goemans and D. Williamson. "A general approximation technique for constrained forest problems". SIAM Journal on Computing, 24:296 - 317, 1995.

[GW95b] M. Goemans and D. Williamson. "Improved approximation algorithms for maximum cut and satisfiability problems using semidefinite programming". Journal of the ACM, 42:1115 - 1145, 1995.

[Gyo78] E. Gyori. "On division of graphs to connected subgraphs". In Combinatorics (Proc. Fifth Hungarian Combinatorial Coll., 1976, Keszthely), pages 485 - 494. Bolyai - North-Holland, 1978.

[Hal95] M. Halldórsson. "Approximating via partitioning". Technical Report IS-RR-95-0003F, JAIST, March 1995.

[Hås01] J. Håstad. "Some optimal inapproximability results". Journal of the ACM, 48:798 - 859, 2001.

[Hoc97] D. Hochbaum, editor. Approximation Algorithms for NP-hard Problems. PWS Publishing Company, 1997.

[IK75] O. H. Ibarra and C. E. Kim. "Fast approximation algorithms for the knapsack and sum of subset problems". Journal of the ACM, 22:463 - 468, 1975.

[Joh74] D. Johnson. "Approximation algorithms for combinatorial problems". Journal of Computer and System Sciences, 9:256 - 278, 1974.

[Kan92] V. Kann. On the Approximability of NP-complete Optimization Problems. PhD thesis, Department of Numerical Analysis and Computing Science, Royal Institute of Technology, S-100 44 Stockolm, Sweden, May 1992.

[Kan94] V. Kann. "Polynomially bounded minimization problems that are hard to approximate". Nordic Journal of Computing, 1:317 - 331, 1994. 
[Kar72] R. Karp. "Reducibility among combinatorial problems". In Proceedings of the Complexity of Computer Computations, pages 85 - 103. Plenum Press, 1972.

[KG98] J. Kleinberg and M. Goemans. "The Lovász theta function and a semidefinite programming relaxation of vertex cover". SIAM Journal on Discrete Mathematics, 11(2):196 - 204, 1998.

[KK82] R. Karp and N. Karmarkar. "An efficient approximation scheme for the one-dimensional bin-packing problem". In Proceedings of the 23rd Annual IEEE Symposium on Foundations of Computer Science, pages $312-320,1982$.

[KMSV99] S. Khanna, R. Motwani, M. Sudan, and U. Vazirani. "On syntatic versus computational views of approximability". SIAM Journal on Computing, 28(1):164 - 191, 1999.

[Kre88] M. Krentel. "The complexity of optimization problems". Journal of Computer and System Sciences, 36:490 - 509, 1988.

[KT94] P. Kolaitis and M. Thakur. "Logical definability of NP optimization problems". Information and Computation, 115(2):321-353, December 1994 .

[KT95] P. Kolaitis and M. Thakur. "Approximation properties of NP minimization classes". Journal of Computer and System Sciences, 50(3):391 - 411, 1995.

[Lar94] I. Lari. "Partizioni ottime di grafi a griglia". PhD thesis, University of Rome "La Sapienza", 1994.

[Law79] E. L. Lawler. "Fast approximation algorithms for knapsack problems". Mathematics of Operations Research, 4(4):339 - 356, 1979.

[LFKN92] C. Lund, L. Fortnow, H. Karloff, and N. Nisan. "Algebraic methods for interactive proof systems". Journal of the ACM, 39(4):859-868, 1992.

[Lov75] L. Lovász. "On the ratio of optimal integral and fractional covers". Discrete Mathematics, 13:383 - 390, 1975. 
[Lov77] L. Lovász. "A homology theory for spanning trees of a graph". In Acta Math. Acad. Sci. Hungar., volume 30, pages 241 - 251, 1977.

[Lov79] L. Lovász. Combinatorial Problems and Exercises. North-Holland Publishing Co., 1979.

[LPS89] M. Lucertini, Y. Perl, and B. Simeone. "Image enhancement by path partitioning". In Proceedings of the Recent Issues in Pattern Analysis and Recognition, volume 399 of Lectures Notes in Computer Science, pages $12-22$. Springer, 1989.

[LPS93] M. Lucertini, Y. Perl, and B. Simeone. "Most uniform path partitioning and its use in image processing". Discrete Applied Mathematics, 42:227 - 256, 1993.

[LR92] H. Lu and R. Ravi. "The power of local optimization: approximation algorithms for maximum-leaf spanning tree". In Proceedings of the 30th Annual Allerton Conference on Communication, Control and Computing, pages 533 - 542, 1992.

[LS97] D. Lapidot and A. Shamir. "Fully parallelized multi-prover protocols for nexp-time". Journal of Computer and System Sciences, 54(2):215 $-220,1997$.

[LY94] C. Lund and M. Yannakakis. "On the hardness of approximating minimization problems". Journal of the ACM, 41:960 - 981, 1994.

[MM94] J. Ma and S. Ma. "An $\mathrm{O}\left(k^{2} n^{2}\right)$ algorithm to find a k-partition in a k-connected graph". Journal of Computer Science and Technology, 9(1):86 - 91, 1994.

[MPS98] E. Mayr, H.-J. Prömel, and A. Steger. Lectures on Proof Verification and Approximation Algorithms. Springer, 1998.

[MRS95] A. Mingozzi, S. Ricciardelli, and M. Spadoni. "Partitioning a matrix to minimize the maximum cost". Discrete Applied Mathematics, 62:221 - 248, 1995 .

[MSN97] A. Maravalle, B. Simeone, and R. Naldini. "Clustering on trees". Computational Statistics and Data Analysis, 24:217 - 234, 1997. 
[NRN97] S. Nakano, M. Rahman, and T. Nishizeki. "A linear-time algorithm for four-partitioning four-connected planar graphs". Information Processing Letters, 62:315 - 322, 1997.

[OM87] P. Orponen and H. Mannila. "On approximation preserving reductions: complete problems and robust measures". Technical Report C - 1987-28, Department of Computer Science, University of Helsinki, 1987.

[PM81] A. Paz and S. Moran. "Non deterministic polynomial optimization problems and their approximations". Theoretical Computer Science, $15: 251-277,1981$.

[PR93] A. Panconesi and D. Ranjan. "Quantifiers and approximations". Theoretical Computer Science, 107:145-163, 1993.

[PS81] Y. Perl and S. Schach. "Max-min tree partitioning". Journal of the ACM, 28(1):5 - 15, 1981.

[PY82] C. Papadimitriou and M. Yannakakis. "The complexity of restricted spanning tree problems". Journal of the ACM, 29(2):285 - 309, 1982.

[PY91] C. H. Papadimitriou and M. Yannakakis. "Optimization, approximation and complexity classes". Journal of Computer and System Sciences, 43:425 - 440, 1991.

[RW02] R. Ravi and D. Williamson. "An approximation algorithm for minimum-cost vertex-connectivity problems". Algorithmica, 34(1):98 $-107,2002$.

[Sal96] L. Salgado. "Aproximabilidade de Problemas de Otimização NPcompletos em Grafos". Dissertação de mestrado, Departamento de Informática, UFPE, Brasil, 1996.

[SG76] S. Sahni and T. Gonzalez. "P-complete approximation problems". Journal of the ACM, 23:555 - 565, 1976.

[Sha92] A. Shamir. "IP = PSPACE". Journal of the ACM, 39(4), 1992. 
[SLPS90] C. De Simone, M. Lucertini, S. Pallottino, and B. Simeone. "Fair dissections of spiders, worms and caterpillars". Networks, 20:323344, 1990.

[STN90a] H. Suzuki, N. Takahashi, and T. Nishizeki. "A linear algorithm for bipartition of biconnected graphs". Information Processing Letters, 33(5):227 - 232, 1990.

$\left[\mathrm{STN}^{+}\right.$90b] H. Suzuki, N. Takahashi, T. Nishizeki, H. Miyano, and S. Ueno. "An algorithm for tripartitioning 3-connected graphs". Journal of Information Processing Society of Japan, 31(5):584 - 592, 1990.

[Sze94] M. Szegedy. "A note on the theta number of Lovász and the generalized Delsarte bound". In Proceedings of the 35th Annual IEEE Symposium on Foundations of Computer Science, pages 36 - 39, 1994.

[Tan96] A. Tanenbaum. Computer Networks. Prentice Hall, third edition, 1996.

[Vaz00] V. Vazirani. Approximation Algorithms. Springer-Verlag, 2000.

[WGMV95] D. Williamson, M. Goemans, M. Mihail, and V. Vazirani. "An approximation algorithm for general graph connectivity problems". Combinatorica, 15:435 - 454, 1995.

[Wil98] D. Williamson. "Lecture notes on approximation algorithms". Technical Report RC 21409, T.J. Watson Research Center (IBM Research Division), Yorktown Heights, New York, 1998.

[WLB ${ }^{+99}$ B. Wu, G. Lancia, V. Bafna, K. Chao, R. Ravi, and C. Tang. "A polynomial time approximation scheme for minimum routing cost spanning trees". SIAM Journal on Computing, 29(3):761 - 778, 1999.

[Zim99] M. Zimand. "Weighted NP optimization problems: logical definability and approximation properties". SIAM Journal on Computing, 28(1):36 - 56, 1999. 



\section{Apêndice}

\section{Max q-Partição Conexa Balanceada}

Instância: um grafo conexo $G=(V, E)$ e uma função $w: V \rightarrow \mathbb{Z}_{+}$. Solução viável: uma $q$-partição conexa $\left(V_{1}, \ldots, V_{q}\right)$ de $G$. Função: $\min \left\{w\left(V_{1}\right), \ldots, w\left(V_{q}\right)\right\}$.

Objetivo: maximizar.

\section{Max Árvore Balanceada}

Instância: um grafo conexo $G=(V, E)$ e uma função $w: V \rightarrow \mathbb{Z}_{+}$. Solução viável: uma árvore geradora $T$ de $G$.

Função: $\max _{e \in E(T)} \mathcal{F}(e, T)$, onde $\mathcal{F}(e, T)=w\left(T_{1}\right) \cdot w\left(T_{2}\right)$, sendo $T_{1}$ e $T_{2}$ as árvores de $T-e$.

Objetivo: maximizar.

\section{$\operatorname{Max}(p / k)$-Bipartição Fracionária Conexa}

Instância: um grafo conexo $G=(V, E)$ e uma função $w: V \rightarrow \mathbb{Z}_{+}$.

Solução viável: uma 2-partição conexa $\left(V_{1}, V_{2}\right)$ de $V$ tal que $w\left(V_{1}\right) \leq(p / k) w(V)$.

Função: $w\left(V_{1}\right)$.

Objetivo: maximizar.

$O B S$ : definido para inteiros $p$ e $k$ tais que $1 \leq p<k$. 


\section{Max Conjunto Independente}

Instância: um grafo $G=(V, E)$.

Solução viável: um subconjunto $V^{\prime} \subseteq V$ tal que quaisquer dois vértices em $V^{\prime}$ são não-adjacentes.

Função: $\left|V^{\prime}\right|$.

Objetivo: maximizar.

\section{Min Cobertura de Vértices}

Instância: um grafo $G=(V, E)$.

Solução viável: um subconjunto $V^{\prime} \subseteq V$ tal que para cada aresta em $E$ pelo menos um de seus extremos pertence a $V^{\prime}$.

Função: $\left|V^{\prime}\right|$.

Objetivo: minimizar.

\section{Max Clique}

Instância: um grafo $G=(V, E)$.

Solução viável: um subconjunto $V^{\prime} \subseteq V$ tal que quaisquer dois vértices em $V^{\prime}$ são adjacentes.

Função: $\left|V^{\prime}\right|$.

Objetivo: maximizar.

\section{Min Caixeiro Viajante}

Instância: um conjunto $C$ de $m$ cidades, distâncias $d\left(c_{i}, c_{j}\right)$ para cada par de cidades $c_{i}, c_{j} \in C$.

Solução viável: uma permutação $\pi:[1 . . m] \rightarrow[1 . . m]$.

Função: $d\left(\left\{c_{\pi(m)}, c_{\pi(1)}\right\}\right)+\sum_{i=1}^{m-1} d\left(\left\{c_{\pi(i)}, c_{\pi(i+1)}\right\}\right)$.

Objetivo: minimizar. 


\section{Min Cobertura de Conjuntos}

Instância: uma coleção $C$ de subconjuntos de um conjunto finito $X$.

Solução viável: um subconjunto $C^{\prime} \subseteq$ Ctalque $\bigcup S\left|S \in C^{\prime}=\bigcup S\right| S \in C$.

Função: $\left|C^{\prime}\right|$.

Objetivo: minimizar.

\section{Min Coloração}

Instância: um grafo $G=(V, E)$.

Solução viável: uma coloração própria dos vértices de $G$.

Função: número cromático de $G$.

Objetivo: minimizar.

\section{Max Corte}

Instância: um grafo $G=(V, E)$.

Solução viável: um subconjunto $V^{\prime} \subseteq V$.

Função: $\mid\left\{\left(v_{1}, v_{2}\right) \in E: v_{1} \in V^{\prime}\right.$ e $\left.v_{2} \in V-V^{\prime}\right\} \mid$.

Objetivo: maximizar.

\section{Min Empacotamento}

Instância: um conjunto finito $U$ de itens, um tamanho $s(u) \in \mathbb{Z}^{+}$para cada $u \in U$, e uma capacidade $B$ inteira positiva do bin.

Solução viável: uma partição de $U$ em conjuntos disjuntos $U_{1}, U_{2}, \ldots, U_{m}$ tais que a soma dos itens em cada $U_{i}$ é menor ou igual a $B$.

Função: o número de bins usados, isto é, o número de conjuntos disjuntos, $m$.

Objetivo: minimizar. 


\section{Max Mochila}

Instância: um conjunto finito $I=\{1, \ldots, n\}$, e para cada $i \in I$ inteiros $a_{i}, p_{i}$, e um inteiro $B$.

Solução viável: um subconjunto $S \subseteq I$ tal que $\sum_{i \in S} a_{i} \leq B$.

Função: $\sum_{i \in S} p_{i}$.

Objetivo: maximizar.

\section{Max 0/1 Mochila}

Instância: um conjunto finito $I=\{1, \ldots, n\}$, para cada $i \in I$ inteiros $a_{i}, p_{i}$, e um inteiro $B$.

Solução viável: uma função $f: I \rightarrow\{0,1\}$ tal que $\Sigma_{i \in I} f(i) a_{i} \leq B$.

Função: $\sum_{i \in I} f(i) p_{i}$.

Objetivo: maximizar.

\section{Min Partição}

Instância: um conjunto finito $X$ de itens, para cada $x_{i} \in X$ um peso $a_{i} \in \mathbb{Z}^{+}$. Solução viável: uma partição dos itens em dois conjuntos $Y_{1}$ e $Y_{2}$.

Função: $\max \left\{\sum_{x_{i} \in Y_{1}} a_{i}, \sum_{x_{i} \in Y_{2}} a_{i}\right\}$.

Objetivo: minimizar.

\section{Max Sat}

Instância: um conjunto finito $U$ de variáveis e um conjunto $C$ de cláusulas disjuntivas de literais.

Solução viável: um subconjunto $C^{\prime} \subseteq C$ tal que existe uma atribuição de valores verdade para $U$ onde toda cláusula em $C^{\prime}$ é satisfeita.

Função: $\left|C^{\prime}\right|$.

Objetivo: maximizar. 


\section{$\operatorname{Max} k$ Sat}

Instância: um conjunto finito $U$ de variáveis e um conjunto $C$ de cláusulas disjuntivas cada uma envolvendo no máximo $\mathrm{k}$ literais, $k \geq 2$.

Solução viável: um subconjunto $C^{\prime} \subseteq C$ tal que existe uma atribuição de valores verdade para $U$ onde toda cláusula em $C^{\prime}$ é satisfeita.

Função: $\left|C^{\prime}\right|$.

Objetivo: maximizar.

\section{Max Exato $k$ Sat}

Instância: um conjunto finito $U$ de variáveis e um conjunto $C$ de cláusulas disjuntivas cada uma envolvendo exatamente $\mathrm{k}$ literais, $k \geq 2$.

Solução viável: um subconjunto $C^{\prime} \subseteq C$ tal que existe uma atribuição de valores verdade para $U$ onde toda cláusula em $C^{\prime}$ é satisfeita.

Função: $\left|C^{\prime}\right|$.

Objetivo: maximizar.

\section{Min Sat}

Instância: um conjunto finito $U$ de variáveis e um conjunto $C$ de cláusulas disjuntivas de literais.

Solução viável: um subconjunto $C^{\prime} \subseteq C$ tal que existe uma atribuição de valores verdade para $U$ onde toda cláusula em $C^{\prime}$ é satisfeita.

Função: $\left|C^{\prime}\right|$.

Objetivo: minimizar.

\section{Max Soma de Subconjuntos}

Instância: um conjunto finito $I=\{1, \ldots, n\}$, para cada $i \in I$ um um valor $a_{i} \in \mathbb{Z}^{+}$ e um inteiro $B$.

Solução viável: um subconjunto $S \subseteq I$ tal que $\sum_{i \in S} a_{i} \leq B$.

Função: $\sum_{i \in S} a_{i}$.

Objetivo: maximizar. 